Portland State University

PDXScholar

5-6-1997

\title{
A Lay Theory of Relational Satisfaction with Best Friends in Japan
}

\author{
Eriko Maeda \\ Portland State University
}

Follow this and additional works at: https://pdxscholar.library.pdx.edu/open_access_etds

Part of the Speech and Rhetorical Studies Commons Let us know how access to this document benefits you.

\section{Recommended Citation}

Maeda, Eriko, "A Lay Theory of Relational Satisfaction with Best Friends in Japan" (1997). Dissertations and Theses. Paper 5315.

https://doi.org/10.15760/etd.7188

This Thesis is brought to you for free and open access. It has been accepted for inclusion in Dissertations and Theses by an authorized administrator of PDXScholar. Please contact us if we can make this document more accessible: pdxscholar@pdx.edu. 
The abstract and thesis of Eriko Maeda for the Master of Science in Speech Communication were presented May 6, 1997, and accepted by the thesis committee and the department.

COMMITTEE APPROVALS:

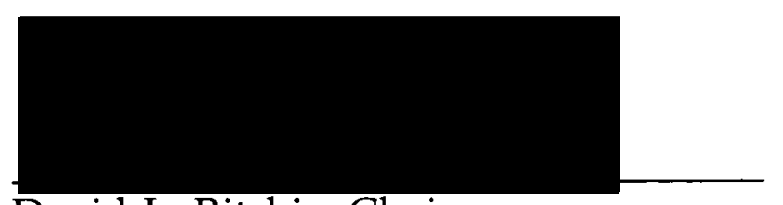

David L. Ritchie, Chair

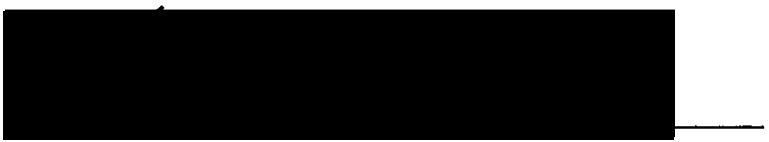

Susan B. Poulsen

DEPARTMENT APPROVAL:

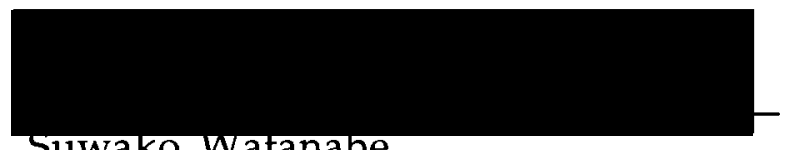

Suwako Watanabe

Representative of the Office of Graduate Studies

Stephen A. Kosokoff, Chair

Department of Speech Communication

\section{ACCEPTED FOR PORTLAND STATE UNIVERSITY BY THE LIBRARY}

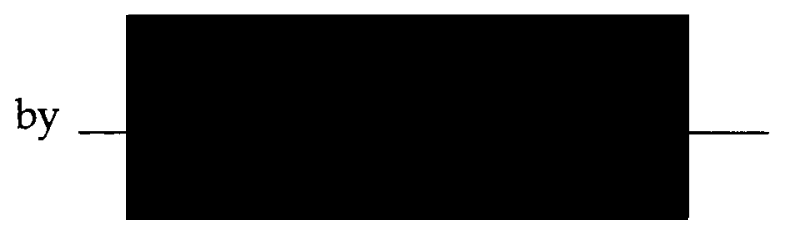

on

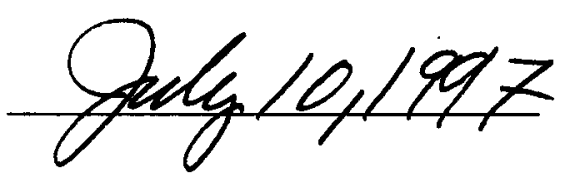




\section{ABSTRACT}

An abstract of the thesis of Eriko Maeda for the Master of Science in Speech Communication presented May 6, 1997.

Title: A Lay Theory of Relational Satisfaction with Best Friends in Japan.

Using a total of 529 Japanese college students (275 females, 254 males) living in Japan, the present study employed five sequential stages. First, it explored Japanese people's beliefs about relational satisfaction with best friends and the organization of these beliefs. In order to explore these beliefs and their organization, this study replicated Cole and Bradac's (1994) study of a lay theory of relational satisfaction with best friends, which focused on people from the United States. The study then examined similarities and differences of Japanese beliefs and organization of the beliefs with those of the U.S. Lastly, the study investigated Japanese gender influence on the beliefs concerning relational satisfaction with best friends.

Thirty-nine Japanese beliefs about relational satisfaction with best friends were identified and multidimensional scaling analyses suggested that these beliefs were organized along three dimensions. These dimensions were related to a best friend's a) interpersonal-social desirable characteristics, b) interdependent-dependent dependability, and c) interpersonal-social demeanor or active-passive support.

Comparison of the beliefs in this study to those of Cole and Bradac's (1994) revealed both similarities and differences of beliefs concerning 
relational satisfaction with best friends between Japanese and U.S. subjects. The differences were explained by Triandis (1986) and others' (e.g., Hofstede, 1984) cultural dimension of individualism-collectivism and Hofstede's (1984) uncertainty-avoidance. Comparison of organization of beliefs between the two studies implied that both Japanese and U.S. cultures shared the dimensions of desirable characteristics and dependability. However, the Japanese organization did not share the U.S. first dimension which related to a best friend's level of activity. This finding was explained by Hofstede's (1984) cultural dimension of uncertainty-avoidance and Kluckhohn and Strodtbeck's (1961) activity orientation.

Exploration of Japanese gender influence on beliefs about relational satisfaction with best friends suggested some shared gender influences across cultures as well as culturally specific gender influences concerning friendships. The culturally specific gender influences were explained by traditional gender roles and recent changes of women's status and their influence on women's perception in the Japanese culture.

In conclusion, the results suggest that Japanese people possess a complex of beliefs about relational satisfaction with best friends and that these beliefs are organized along three dimensions. The results also suggest that there are both similarities and differences concerning relational satisfaction with best friends between Japan and the U.S. 


\title{
A LAY THEORY OF RELATIONAL SATISFACTION WITH BEST FRIENDS IN JAPAN
}

$$
\text { by }
$$

ERIKO MAEDA

A thesis submitted in partial fulfillment of the requirements for the degree of

\author{
MASTER OF SCIENCE \\ in \\ SPEECH COMMUNICATION
}

Portland State University

1997 
uede[ u! słuəsed Ku

o I 


\section{ACKNOWLEDGMENTS}

First, I would like to thank Dr. Ritchie for his persistent help and support both academically and psychologically. His generous support made me possible to complete this thesis. I am also grateful to Dr. Poulsen for her thorough insight, especially in the issue of interpersonal and intercultural communication, as well as personal support. I would like to thank Dr. Watanabe for her insights in Japanese culture and JapaneseEnglish language issue. I am also thankful for all the instructors and professors in Japan who allowed me to use their class time for my study as much as for all the subjects who participated in the study.

I would especially like to thank Masami Nishishiba, Jay Peterson, Nariyo Kono, Nobuko Higashi, Noriko Nakano, Asako Takio, Yuki Dooley, Jack Horner, James Wilkins and many other friends for their precious help and encouragement.

Lastly, I would express my appreciation to my family and friends in Japan who have fully and continuously support me from Japan. 
TABLE OF CONTENTS

PAGE

ACKNOWLEDGMENTS $\ldots \ldots \ldots \ldots \ldots \ldots \ldots \ldots \ldots \ldots \ldots \ldots \ldots \ldots$ iii

LIST OF TABLES $\ldots \ldots \ldots \ldots \ldots \ldots \ldots \ldots \ldots \ldots \ldots \ldots \ldots \ldots \ldots \ldots \ldots$

LIST OF FIGURES $\ldots \ldots \ldots \ldots \ldots \ldots \ldots \ldots \ldots \ldots \ldots \ldots \ldots \ldots \ldots \ldots \ldots \ldots \ldots$ CHAPTER

I INTRODUCTION $\ldots \ldots \ldots \ldots \ldots \ldots \ldots \ldots \ldots \ldots \ldots$

Purpose of the Study $\ldots \ldots \ldots \ldots \ldots \ldots \ldots \ldots 1$

Justification of the Study .............. 2

Research Questions .................6

II LITERATURE REVIEW $\ldots \ldots \ldots \ldots \ldots \ldots \ldots \ldots \ldots$

Lay Theories $\ldots \ldots \ldots \ldots \ldots \ldots \ldots \ldots \ldots, 8$

Friendship $\ldots \ldots \ldots \ldots \ldots \ldots \ldots \ldots \ldots \ldots \ldots$

Culture ....................... 27

III $\quad$ STAGE ONE $\ldots \ldots \ldots \ldots \ldots \ldots \ldots \ldots \ldots \ldots \ldots \ldots \ldots$

Purpose $\ldots \ldots \ldots \ldots \ldots \ldots \ldots \ldots \ldots \ldots \ldots, 41$

Method $\ldots \ldots \ldots \ldots \ldots \ldots \ldots \ldots \ldots \ldots, 43$

Results....................... 53

Discussion ...................... 56

Conclusion ...................... 64

IV STAGE TWO ........................ 67

Purpose........................ 67

Method $\ldots \ldots \ldots \ldots \ldots \ldots \ldots \ldots \ldots \ldots, 68$

Results........................... 73 
Discussion ..................... 81

Conclusion ................... 85

V STAGE THREE .................... 87

Purpose ...................... 87

Method ..................... 88

Results ..................... 92

Discussion ...................... 93

Conclusion .................... 97

VI STAGE FOUR .................... 99

Purpose $\ldots \ldots \ldots \ldots \ldots \ldots \ldots \ldots \ldots \ldots \ldots 9$

Method ......................... 100

Results ........................ 103

Discussion ...................... 105

Conclusion ...................... 110

VII STAGE FIVE $\ldots \ldots \ldots \ldots \ldots \ldots \ldots \ldots \ldots \ldots \ldots \ldots$

Purpose ...................... 113

Method ...................... 114

Results........................ 119

Discussion .................... 125

Conclusion ..................... 132

VIII CONCLUSION ........................ 136

Summary of the findings $\ldots \ldots \ldots \ldots \ldots \ldots$

Limitations and Strengths of the Study . . . . . . . 139

Future Directions .................. 141

REFERENCES ............................. 144 


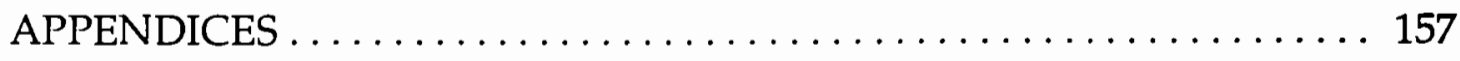

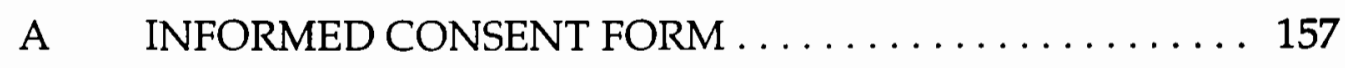

B ENGLISH TRANSLATION OF

THE QUESTIONNAIRES ................. 159

C INSTRUCTION FOR STAGE ONE $\ldots \ldots \ldots \ldots \ldots \ldots \ldots$

D PERSONAL INFORMATION SURVEY ......... 176

E COLE AND BRADAC'S (1994) PERCEIVED BELIEFS ABOUT RELATIONAL SATISFACTION WITH BEST FRIENDS . . 178

F QUESTIONNAIRES AND PERSONAL INFORMATION

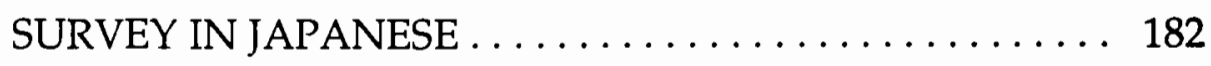




\section{LIST OF TABLES}

TABLE

PAGE

3-1 Japanese Perceived Beliefs of Relational Satisfaction with Best Friends . . . . . . . . . . . . . . . . . 54

3-2 Similar Categories of Relational Satisfaction with Best Friends: Japan and the United States . . . . . . . 57

3-3 Different Categories of Relational Satisfaction with Best Friends: Japan and the United States . . . . . . . . 59

4-1 Descriptions of the Seven Clusters . . . . . . . . . 77

5-1 Regression Results for Likert-Scale Ratings over Proposed Dimensional Interpretations . . . . . . . . . 93

6-1 Similarity between the Japanese and U.S. Beliefs about Relational Satisfaction with Best Friends . . . . . . . . 102

6-2 Regression Results for U.S. Dimensions over Japanese Dimensions . . . . . . . . . . . . . . . . . . 104

6-3 Regression Results for Japanese Dimensions over U.S. Dimensions ........................... 105

6-4 Comparison of Interpretations between Japanese and U.S. Underlying Dimensions . ................. 106

7-1 Descriptions of the Seven Clusters . . . . . . . . . 117

7-2 Means and Standard Deviations for Number of Words Chosen from Each Cluster . . . . . . . . . . . . . . 120 


\section{LIST OF FIGURES}

\section{FIGURE}

PAGE

4-1 Stress values along one to six dimensions of Japanese people's organization of beliefs about satisfaction with best friends. . . . . . . . . . . . . . . . . 74

4-2 Dendrogram of the clusters of Japanese people's beliefs about relational satisfaction with best friends. . . . . . 75

4-3 Coefficient of agglomeration schedule in order to uncover subgroups of Japanese beliefs about satisfaction with best friends. . . . . . . . . . 76

4-4 Dimension 1 and 2 in the three-dimensional solution of Japanese people's beliefs about relational satisfaction with best friends.

4-5 Dimension 1 and 3 in the three-dimensional solution of Japanese people's beliefs about relational satisfaction with best friends. . . . . . . . . . . . . . . 80 


\section{Chapter I}

\section{Introduction}

This chapter describes the overview of the present study. The chapter consists of three sections: the purpose of this study, justification, and research questions.

\section{Purpose of the Study}

There are two main purposes in this study. One, to explore Japanese people's beliefs about relational satisfaction with best friends. Two, to examine similarities and differences concerning people's perceived beliefs about relational satisfaction with best friends between Japan and the United States.

In order to accomplish the purposes above, the study includes five sequential stages. Each stage serves a different aspect of the purposes. Specifically, the purpose of stage one is to explore the Japanese people's beliefs about relational satisfaction with best friends. Stage two investigates the organization of Japanese people's beliefs about relational satisfaction with best friends. Stage three assesses interpretations of underlying dimensions or organization of beliefs about relational satisfaction with best friends. The design of these three stages is a replication of Cole and Bradac's (1994) study on a lay theory of relational satisfaction with best friends, which focused on people from the United States. 
Stage four attempts to compare underlying dimensions of beliefs of Japanese about relational satisfaction with best friends to those of Cole and Bradac (1994). Stage five explores gender influence on Japanese people's beliefs about relational satisfaction with best friends. While Cole and Bradac examined gender influence on best friendship, this study does not use the same method as they did. Therefore, this stage is not comparable to Cole and Bradac's study.

Justification of the Study

There are growing numbers of Japanese living in the United States; 258,300 Japanese were reported to be living in North America in 1990 (Keizai Koho Center, 1992). According to the U.S. Immigration and Naturalization Service $(1985,1994)$, the number of Japanese students studying in the U.S. increased from 14,542 in 1984 to 40,492 in 1993, which is a more than $175 \%$ increase in less than a decade.

Living in a foreign culture tends to cause stress due to various factors such as language problems, separation reactions, misunderstandings and loneliness (Oberg, 1960). For example, Furnham and Trezise (1983) reported that international students from Africa, Middle East, Europe and Malaysia showed significantly more psychological disturbance than either British control group or British first-year students regardless their different cultural backgrounds.

While it is a stressful situation, social interaction with the host culture is important in order to adjust to a new environment (Brein \& 
David, 1971). There are many ways to interact with the host culture socially such as listening to the radio, studying with host members or going to a host member's party. Sewell and Davidsen (1960) reported that Scandinavian students who had more social interaction with Americans felt more satisfaction. In the study of intercultural competency, Taylor (1994) identified three categories as behavioral learning strategies for sojourners; observer, participant, and friend. Of these three categories, he suggested that development of close friendships with members of a host culture is the most significant factor for sojourners and their development of intercultural competency. Therefore, it may be crucial for Japanese who are living in the U.S. to develop friendships with people from the U.S. in order to have positive intercultural experiences.

However, many Japanese living in the United States have reported difficulties in establishing friendships with U.S. people. For instance, Trice and Elliott (1993) reported that Japanese students in the U.S. preferred spending time with their home nationals in various activities such as studying and attending social activities: they spent at least $88 \%$ of their study time and $82 \%$ of their social time with other Japanese students.

While many factors may account for why Japanese people have difficulties establishing intercultural friendships (e.g., their language ability, expectations, and personality), the present study attempts to examine whether people from Japan and the U.S. have the same ideas about friendship. If people from the two cultures have different ideas about friendship, those differences may hinder the development of intercultural friendships. As a result, in addition to losing opportunities to 
enrich their intercultural experiences, Japanese people in the U.S. may construct biases about the U.S. culture and its people.

While many studies have examined friendships, they have mainly focused on middle-class Caucasian people living in the United States (Adams \& Blieszner, 1994) by U.S. researchers. Review of Japanese friendship literature revealed that some have examined friendships in children or adolescents (e.g., Nakayama, 1992; Shima, 1994) and others have focused on a specific aspect of friendship such as perception of equity on friendship (Matsuura, 1991) and self-disclosure patterns in friendships (Enomoto, 1987). I have not found studies focused directly on core friendship concepts of Japanese adults. There are some cross-cultural studies related to friendships. However, they also examined a specific aspect of friendship such as testing uncertainty reduction theory in the three cultures of Japan, Korea, and the U.S. (Gudykunst, Yang, and Nishida, 1985) and the rules of relationships, including friendships, crossculturally (Argyle \& Henderson, 1985). In short, the review of literature indicates a need for examining the concept of Japanese adult friendships.

This study may have a significant meaning not only for Japanese but also for people from the U.S living in Japan. In 1993, 507,391 people from the U.S. entered Japan (Ryoji Ijubu, 1994). According to the International Herald Tribune, "More than one-third of all Americans who take up residence in foreign countries returns prematurely because they are unable to adapt to day-to-day life" (cited in Storti, 1990, p. xiii-xiv). It is suspected therefore that many U.S. people living in Japan also have difficulty 
adjusting to the Japanese culture. For those people, development of friendship may also be the key to successful intercultural experiences.

The present study focused on the concept of "best friend" instead of that of "friend" for two reasons. First, since a part of this study is a replication of Cole and Bradac's (1994) study which examines U.S. people's relational satisfaction with best friends, it is important to be consistent with their study. Second, "friend" is an ambiguous term and its ambiguity often creates research problems (Adelman, Parks \& Albrecht, 1990; Caroline, 1993). For instance, Caroline (1993) states that "it is unknown what kinds of friendships the respondents are reporting and whether the types of friendships ... have an impact on the results of the research" (p. 236). It seems that more precise description of the concept such as "casual," "good," or "true" friendships helps to reduce ambiguity of the term. Rawlins, Leibowitz, and Bochner (1986) state that "a person's 'best-friend' serves as a baseline by which he or she compares or differentiates other friendships." Therefore, this study examined a concept of relational satisfaction with "best friends."

This study is grounded in a lay theory approach. This approach suggests that people develop theories or have explanations for their daily phenomena in order to make sense of the way the physical and social world works (Furnham, 1988). Exploring Japanese lay theory of satisfaction with best friends may help us understand the way Japanese people behave as well as predict their behaviors with their friends. In addition, by replicating Cole and Bradac's (1994) study, this study may reveal cultural 
influences on lay people's beliefs about satisfaction with their best friends, specifically differences between Japanese and U.S. cultures.

\section{Research Questions}

The present study first examines Japanese people's beliefs about relational satisfaction with best friends. Since the first three stages of this study are a replication of Cole and Bradac's (1994) study, the study then explores similarities and differences concerning relational satisfaction with best friends between Japan and the United States. The following research questions are addressed:

RQ1: What do Japanese people report as beliefs about satisfaction with best friends?

RQ2: How similar are beliefs about satisfaction with best friends between Japan and the United States?

RQ3: How do Japanese people organize these beliefs about satisfaction in their minds?

RQ4: How similar is organization of beliefs about satisfaction with best friends between Japan and the United States?

This study also examins gender influence on Japanese people's beliefs about relational satisfaction with best friends. The following research questions are addressed for the gender influence on this study: RQ5: How does people's own gender influence their beliefs about satisfaction with best friends in Japan? 
RQ6: How does people's best friend's gender influence their beliefs about satisfaction with these friends in Japan? 
Chapter II

\section{Literature Review}

In this chapter, I review literature relevant to the present study. There are three sections in this chapter: first, I describe the lay theories approach which was used as theoretical framework in this study. Second, I present a review of literature on friendship. Lastly, I introduce cultural variables which seem relevant to the present topic "friendship" and which contrast Japan with the United States.

\section{Lay Theories}

The present study is grounded in the lay theories approach. According to Furnham (1988), this approach suggests that:

in an attempt to make sense of the social and physical world to see it as stable, orderly, predictable and understandable, people develop theories or arrive at explanations for phenomena salient to their lives. Through observation, exposure to others, the media and personal experience people become familiar with "how things work." (p.19)

While people have theories or explanations for social and physical phenomena, these theories may differ from scientific theories. Furnham (1988) introduced the following eight criteria in order to clarify lay theories from scientific theories; (1) explicitness and formality, (2) coherent and 
consistent, and (3) falsification vs verification, (4) cause and consequence, (5) content vs process, (6) internal vs external, (7)general vs specific, and (8) strong vs weak. It is important to note that these criteria are not true to all scientific theories or lay theories. In addition, although these criteria seem to present good scientific theories vs poor lay theories, neither is good or poor, or right or wrong. Instead, Furnham intended to point out that these two different kind of theories merely differ in focus.

\section{Explicitness and Formality}

While scientific theories are usually explained in a formal and explicit manner, lay theories are often implicit. Although lay people hold their beliefs about the way the social and physical world works, they can rarely explain their beliefs in an explicit formal way. In addition, they often do not know what their explanations are derived from.

\section{Coherent and Consistent}

Scientific theories are usually explained in both coherent and consistent ways because they "usually apply to a specific domain of phenomena and make propositions that 'fit together'" (Furnham, 1988, p. 3). By contrast lay theories often seem ambiguous, incoherent, and inconsistent. Lay people may hold two mutually incompatible contradictory beliefs. While some of these inconsistencies may be resolved at a specific level, others remain inconsistent without lay people being aware of its inconsistency.

\section{Falsification vs Verification}

While the initial stage of scientific research is induction, scientific theories usually rely on principles of deductivism once theories have 
developed. Since the goal of deductivism is to disprove theories, falsification is the criterion of science. Lay theories, on the other hand, usually rely on inductivism. Lay people search for verification; they accumulate evidence and make inferences.

\section{Cause and Consequence}

Lay theories are frequently correlational, hence, cannot infer cause. However, lay people often perceive a relationship between two variables and infer unidimensional cause. This is often the case of scientific theories as well.

\section{$\underline{5 . \text { Content vs Process }}$}

While scientific theories are often explanatory and process oriented, lay theories tend to be descriptive and content oriented. This is because "description often precedes explanation and lay theory frequently precedes scientific theory" (Furnham, 1988, p. 5).

\section{Internal (individualistic) vs External (situational)}

Ross (1977) explained the fundamental attribution error as follows; when lay people explain other people's behaviors, they often infer those people's personal dispositions instead of situations. In the case of scientific theories, inference differs depending on focus of the field. For instance, psychologists tend to infer individual behavior in terms of personality and other internal motives while sociologists attempt to explain in terms of sociological forces.

\section{General vs Specific}

While some scientific theories explain various human behaviors based on abstract concepts, lay theories explain specific phenomena based 
on lay people's experiences, expectations, and motivations. Lay people do not generalize their theories to abstract principles but have "smaller 'minitheories' for very specific events" (Furnham, 1988, p. 6).

\section{Strong vs Weak}

Eysenck (1960) distinguished between strong and weak theories in science. Strong theories are based on numerous accurate observations made by different people, have relatively clear cut phenomena, and have straightforward precise predictions. Weak theories do not have the above characteristics. However, because they often do not have precise, accurate data, weak theories may direct research to new problematic areas. While there are some strong theories in the field of social science, most lay theories are considered to be weak theories.

Although lay theories and scientific theories have quite different natures, both kinds of theories are systematic explanations for "the observed facts and laws that relate to a particular aspect of life" (Babbie, 1990, p. 55). In this sense, lay theories and scientific theories have the same aim with different approaches. After all, as Furnham (1988) explains, "lay theories overlap with scientific theories; they function in similar ways . . . [and] the one may be seen as an outgrowth of the other" (p.7).

While lay theories have rarely been explored in the fields of communication or human relationships (Cole and Bradac, 1994), they have been examined in a variety of other disciplines such as psychology, medicine, economics, statistics and law (Furnham, 1988). Previous research on lay theories have demonstrated that people have specific and integrated ideas about the social and psychological phenomena such as 
loneliness (Lunt, 1991), personal debt (Lunt, \& Livingstone, 1991), and unemployment (Furnham, \& Hesketh, 1987).

Because lay theories have a different focus from scientific theories, the study of lay theories can contribute to scientific research and vice versa. In fact, previous research on lay theories has identified important factors which were uncovered by the scientific approach. For instance, in the study of intelligence, lay people valued the importance of social competence more than experts, while experts valued the importance of motivation more than lay people (Sternburg, Conway, Ketron, \& Bernstein, 1981). In the study of crime, Furnham (1988) summarized that lay theories are crime-specific, therefore, "general theories or explanations do not apply equally to all crimes and delinquent acts" (p. 188).

Previous research has reported cultural differences on lay theories in different areas. It is widely known that culture has a strong impact on the belief about health and illness (Helman, 1984; Herzlich, 1973). Cultural differences in lay people's understanding and definition of the nature of intelligence were also reported (e.g., Gill \& Keats, 1982; Klein, Freeman, \& Millett, 1983). Keller, Miranda, and Gauda (1984) found many differences between German and Costa Rican mothers' beliefs about child development.

Using the framework of lay theories, the present study examines Japanese perceived beliefs about relational satisfaction with best friends. Exploring description of beliefs about satisfaction with best friends and organization of these beliefs may reveal criteria for Japanese lay people in order to have a satisfying friendship. In addition, these descriptions and 
the way of organization may help to explain the meaning of people's behaviors toward their friends and their non-friends and to predict people's perceived relationships through their behaviors. Finally, since this study is a replication of Cole and Bradac's (1996) study, comparison of the two studies highlights cultural factors on a lay theory of relational satisfaction concerning best friends between Japan and the U.S.

\section{Friendship}

In this section, I introduce the review of literature on friendship relevant to the present study. There are three parts: the first part discusses about previous research on friendship in general. The second part specifically discusses gender influence on friendship. The last part introduces the summary of Cole and Bradac's (1994) findings relevant to the present study.

\section{Literature on friendship}

Researchers, over the years, have explored people's beliefs related to friendships. Some researchers studied friendship as a subset of personal relationships; others examined its concept. Although friendships are a core aspect of our lives, it seems that no one can define what it is (Fehr, 1996). Some researchers have reported difficulty in defining friendship (e.g., Adelman, Park, \& Albecht, 1990; Caroline, 1993). Adelman et al. (1990) described friendship as "a slippery concept" because it is "both a type of relationship and a quality that people attribute to other types of 
relationships" (p. 284). For instance, when someone says "My mom is my best friend," this statement describes a high quality of mother-child relationship. At the same time, when someone says "He is such a good friend. He is like a part of my family," this describes a high-quality of friendship that can be interpreted in a familial sense. In this sense, friendship is not a mutually exclusive relationship. Moreover, the review of friendship literature offered insight into beliefs people posses concerning friendships.

Some researchers have compared friendship with other personal friendships. Wish, Deutsch, \& Kaplan (1976) examined people's underlying perceptions of interpersonal relations and found four dimensions: 1) cooperative and friendly us competitive and hostile, 2) equal vs unequal,3) intense vs superficial, and 4) socioemotional and informal vs task-oriented and formal. Along these dimensions, close friendships appeared cooperative and friendly, equal, intense, socioemotional, and informal when compared to most other kinds of relationships such as those between teacher and young pupil or those between casual acquaintances.

Rands and Levinger (1979) explored people's expected behaviors in four relationships (casual acquaintances, good friends, close relationship, and married), and found two dimensions: high-low behavioral interdependence and high-low affective interdependence. The first dimension includes items such as "plan a joint project" or "ask for advises." The second dimension includes items such as hugging and standing close to each other. As a relationship moves from casual 
acquaintances to married, people rated both behavioral and affective interdependence higher. However, since the affective interdependence dimension represents physical contact, the ratings varied depending on the gender of the dyad. For instance, cross-sex pairs tended to have higher affective independence compared to same-sex pairs. In short, when compared to most other personal relationships, friendship is perceived as more imitate, equal and informal.

While comparative studies of personal relationships have highlighted some qualities of friendship relative to other relationships, they do not reveal qualities which are specific to friendship. Some researchers have identified some of the qualities which seem unique to friendship. They are: (1) voluntariness (e.g., Bell, 1981; Rubin, 1986), (2) reciprocity (e.g., Bell, 1981; Devito, 1995), and (3) width of its possible formation (e.g., Caroline, 1993; Pogrebin, 1987). As every person is unique, every friendship is varied (Reohr, 1991). Therefore, some of the following qualities may not apply in describing a particular friendship. However, they do seem to describe qualities of friendship in general.

\section{Voluntariness}

Voluntariness is considered the most distinctive and significant feature of friendship by many U.S. researchers (e.g., Adams \& Bliezner, 1994; Bell, 1981; Devito, 1995; Duck, 1983). Rubin (1986) stated that "friends choose to do what kin are obliged to do. With friends, we must earn the rights and privileges that with family usually come just being part of the collectivity" (p. 22). Because friendship is a choice, whether or not it occurs depends upon the person's ability to commit. Some researchers reported 
that adults in mid-life felt unable to initiate and sustain another relationship because many of them already have engaged in several relationships. (Hays, 1988; Palisi \& Ransford, 1987).

As there is a choice whether to develop friendship, there is also a choice in whether to terminate it. Friendship is more easily terminated than family and kin relationships (Adelman, et al., 1990). In other words, friendship has a limit while kin or family are seen as those you can depend on without limit (Rubin, 1986). Devito (1995) expresses this limitation of friendship as follows:

Friendships must be mutually productive; this qualifier emphasizes that, by definition, they cannot be destructive either to oneself or to the other person .... Lover relationships, marriage relationships, parent-child relationships, and just about any other possible relationship can be either destructive or productive. But friendship must enhance the potential of each person and can only be productive. (pp. 422-423)

Although there is a voluntary quality in choosing to develop or terminate friendship, friendship choices are not wholly fortuitous (Allan, 1989). For example, freedom of choice may be bounded by physical constraint. When you live in a small village, your choice of friends is restricted within the members of the village because these members are the only ones you can contact on a daily basis (Moghaddam, Taylor, \& Wright, 1993).

\section{Reciprocity}

Bell (1981) stated the idea of reciprocity as follows: 
There must always be a quality of exchange between friends. While who gives what and how much relative to the other may often vary in different friendships or even in a given friendship over time, it can not be too one way (p. 403).

The reciprocity between friends is often an abstract sharing such as "helping each other in time of need, listening to each others' problems, and understanding and protecting each other" (Synder \& Smith, 1986, p. 68), although material objects can be exchanged as well. For close friendship, reciprocal exchange includes love and intimacy (Devito, 1995). According to Timmerman (1991), intimacy is " a quality of a relationship in which the individuals must have reciprocal feelings of trust and emotional closeness toward each other and are able to openly communicate thoughts and feelings with each other" (p. 19).

\section{Width of its Possible Formation}

Friendship can be differentiated from other relationships by "the width of its possible formation across the life span" (Caroline, 1993, p. 239). While some relationships such as those between workers and parents are constrained by age (Caroline, 1993), there is the opportunity of forming a new friendship throughout one's life (Pogrebin, 1987). Pogrebin (1987) stated that "in the trinity of human priorities, friendship usually stands behind love and family. Yet, in old age, when new lovers are rare, new children rarer, and the family dwindling, there is, still and always, the possibility of a new friend" ( p. 368).

Some researchers have explored beliefs people possess regarding friendships. Argyle and Henderson (1985) defined friends as "people who 
are liked, whose company is enjoyed, who share interests and activities, who are helpful and understanding, who can be trusted, with whom one feels comfortable, and who will be emotionally supportive" (p. 64). In their study of prototype of friendship, Davis and Todd (1985) identified important factors for friendship: equal eligibilities, respect, trust, acceptance, mutual assistance, enjoyment, spontaneity, understanding, and intimacy.

La Gaipa (1977) identifies six major factors related to friendship. They include: self-disclosure, authenticity, helping behavior, positive regard, strength of character, and similarity. His results suggested that all factors other than strength of character become more important when the level of friendship increases. Especially, self-disclosure and helping behavior seemed important when the relationship is defined as best friends.

Rawlins, Leibowitz and Bochner (1986) examined different kinds of friendships (best, equal, one-up, and one-down friendships) and found that friendships were differentiated along two dimensions: affect and instrumentality. They also found that best friends were rated significantly higher on social intimacy, and suggested that a best friendship might be used as reference to judge intimacy in other friendships.

In their study on the rules of friendship, Argyle and Henderson (1985) reported behaviors which are considered the most detrimental to friendship. They are jealousy or criticism, lack of tolerance for third parties, disclosing confidences, not volunteering help when needed, nagging or public criticism, failing to show trust or share confidences, failing to show positive regard, and failing to provide emotional support. 
They found that high-quality friendships applied more intimacy-reward type rules such as discussing intimate topics and emotional support than do low-quality friendships.

In short, people's beliefs related to friendship include qualities such as enjoyment, sharing things, ,self-disclosure, trust, understanding, and help. While most of these beliefs about friendship become more important as friendship develops, there are two qualities which are particularly important in close friendships; they are (1) equality in social status and (2) duration of relationship.

Equality in social status is a major factor in close friendship (Verbrugge, 1977). Although friendship is viewed as an equal to other relationships, some researchers have stated that less developed friendships were not always equal (e.g., Adeleman, et al., 1990; Rawlins, et al., 1986). Also, close friends need to share the perception that they are socially equal (Reisman, 1979, 1981).

Duration of relationship is also important for close friendship because it is viewed as the history of friendship (Young, 1986). Synder and Smith (1986) stated that friendships were seen as deep if they are "longterm enduring friendships that had weathered hardships and change" (p. 68). This idea of duration implies that spending some period of time including difficult time together helps friendships develop into a higher quality one.

While many studies have examined friendships, they have focused mainly on middle-class Caucasian people living in the United States (Adams \& Blieszner, 1994) by U.S. researchers. Review of Japanese 
friendship literature revealed that very few empirical studies have been done regarding Japanese friendships. Some studies have examined friendships in children or adolescents (e.g., Nakayama, 1992; Shima, 1994) and others have focused on a specific aspect of friendship such as selfdisclosure (e.g., Enomoto, 1987; Endoh, 1995).

Matsuura (1991), using equity theory to interpersonal relations (Homans, 1961), examined the perception of equity on friendship. In his study, dyads in which both parties perceived their relationships as equal were found to be most stable compared to other unequally perceived dyads. Moroi (1989) also studied equity theory and emotional states in same-sex friendships. He found that subjects who perceived themselves as equally treated in the relationship felt more content than those who did not. In short, it seems that perceived equality is an important quality for Japanese friendships. However, I have not found studies focused directly on core concept of Japanese adult friendships.

There have been some cross-cultural studies related to friendships. Some examined various personal relationships including friendships (e.g., Argyle \& Henderson, 1985), while others explored a specific aspect of friendship such as taboo topics (Goodwin \& Lee, 1994) and self-disclosure (Won-Doornink, 1985) rather than people's general beliefs related to friendships. Gudykunst, Yang, and Nishida (1985) tested a model of uncertainty reduction theory across three relationships (acquaintances, friends, and dates) in the three cultures of Japan, Korea, and the U.S. The results suggested that the model was reasonably fit to the data for all 
relationships in the all cultures. However, there were no findings specifically accounting cross-cultural friendships in their study.

British researchers, Argyle and Henderson (1985) extended their study on the rules of relationships to Japan, Hong Kong and Italy. They found that most universal rules for British subjects were not consistently endorsed across all cultures. For instance, they found that Japanese subjects had lower scores on intimacy related rules (e.g., acknowledging birthdays, inviting to family celebrations). However, since the focus of this study was to examine generalizability of rules across relationships in the four cultures, there is not much specific information related to crosscultural friendships.

In short, the review of literature indicates lack of examination of beliefs concerning Japanese friendships as well as cross-cultural comparison related to friendships. In addition, previous cross-cultural research on friendship tended to use questionnaires or items in the questionnaire constructed in a single culture (e.g., Argyle and Henderson, 1985; ). This approach may overlook some qualities which are culturally specific. Therefore, the present study, asks Japanese lay people about their beliefs regarding friendships in order to identify Japanese beliefs about satisfaction with best friends.

\section{Gender Influence on Friendship}

For over twenty years, researchers have studied gender influence on friendships in the United States. In the study of same-sex friendship, some similarities between women's and men's friendships were reported. For 
instance, both women and men value intimate friendships (Caldwell \& Peplau, 1982), are similar in terms of values and expectations (Sherrod, 1989), and consider talking as a primary purpose for getting together with friends (Duck \& Wright, 1993).

At the same time, differences between women's and men's friendships were reported. Compared to men's friendships, women's friendships reflect greater emotional involvement (Bell, 1981; Caldwell \& Peplau, 1982; Black \& Angelis, 1975; Williams, 1985; Weiss \& Lowenthal, 1975; Rubin, 1973), involve more intimate self-disclosure (Johnson \& Aries, 1983; Aries \& Johnson, 1983; Walker \& Wright, 1976; Hacker, 1981), stress reciprocity more (Reisman, 1981; Weiss \& Lowenthal, 1975) and demonstrate significantly more affectionate behaviors (Hays, 1989; Rands \& Levinger, 1979). Compared to women's friendships, men's friendships emphasize sharing activities (Caldwell \& Peplau, 1982; Aukett, Ritchie, \& Mill, 1988, Barth \& Kinder, 1988) and stress commonality (Weiss \& Lowenthal, 1975).

Comparative studies on same- and opposite-sex friendships have yielded mixed results. While Larwood and Wood (1977) found that both females and males prefer same-sex close friendships over opposite-sex friendships, others (Rose, 1985; Rubin, 1985) found that men tend to have more emotional sharing, disclose themselves, and be more intimate with female friends than male friends. Some research indicated that women tend to regard their same-sex friendships as closer and more satisfying than their opposite-sex friendships (Rose, 1985; Rubin, 1985), other reported that 
women's friendships with other women are equally satisfying as those with men (Elkins \& Peterson, 1993; Pleck, 1975).

While more research is needed, especially examining opposite-sex friendships, most of these previous studies of gender influence on friendships were done in the U.S. mainly drawing samples from the dominant white population (e.g., Elkins \& Peterson, 1993; Davidson \& Duberman, 1982; Rose, 1985). Only a few studies investigating gender influence on friendships or related issues have compared different cultures. Lin and Rusbult (1995) examined the commitment process in opposite-sex friendships in the U.S. and Taiwan. They found little evidence of cultural differences in the commitment process. Dion \& Dion (1993) examined influence of gender and ethnocultural background in styles of love. They found consistent gender differences across cultures. Women viewed love as more friendship-oriented, more pragmatic, less permissive than did men. At the same time, they found some cultural differences in gender-role differentiation. Women from Asian ethnocultural backgrounds other than Chinese expressed a more altruistic view of love than did Anglo-Celtic women, and were less likely to view love as a game than either Asian men or their female counterparts in other cultures. Because of very limited number of the cultural comparative studies, cultural differences in gender influence on friendships are unknown.

There have been a few studies related to Japanese adult friendships. Enomoto (1987) studied Japanese self-disclosure patterns. According to him, both male and female subjects showed significantly more self- 
disclosure to their same-sex best friends than opposite-sex best friends. In a study employing equity theory to analyze same-sex friendships, Moroi (1989) found that the feeling of equity is more important for male subjects when friendship develops, whereas the feeling of equity is more important for females in the early stages of friendship. Since the number of studies is very limited and most studies focused on a specific aspect of friendship, no generalization can be made concerning gender influence on Japanese friendships.

In order to gain a better understanding of gender influence in general and cultures in particular, stage five of the present study examines gender influence on best friendships in Japan. Specifically, stage five examined whether Japanese men and women have different emphases in their beliefs about satisfaction with best friends, depending on their own gender and/or their best friend's gender.

\section{Summary of Cole and Bradac's (1994) Study}

The first three stages of this study replicated a part of Cole and Bradac's (1994) study on people's lay beliefs about relational satisfaction with best friends. By using U.S. college students as a sample, Cole and Bradac investigated (1) people's perceived beliefs about satisfaction with best friends, (2) underlying dimensions of those beliefs, and (3) causal structure of the beliefs. The present study addressed the first two questions by using Japanese college students as a sample. Therefore, the findings and discussions of the first two questions in Cole and Bradac's study are introduced here. 
Forty-three perceived beliefs about satisfaction with best friends were identified (see Appendix E). According to Cole and Bradac (1994), these 43 beliefs constitute a diverse set including qualities of communication (e.g., approachable, honest), specific communicative behaviors (e.g., admits mistakes, not a whiner), physical features (e.g., healthy, physically attractive), and personal traits (e.g., ambitious, outgoing). In short, Cole and Bradac claim that communication plays a central role in people's beliefs about satisfaction with best friends and those beliefs are related to positive outcomes.

In their findings concerning 43 beliefs about satisfaction, Cole and Bradac (1994) found that their subjects express concern regarding abuse and violence. Since this concern has not been reported by any previous research on friendships, Cole and Bradac proposed the following possible interpretations. First, the results may be due to the way Cole and Bradac posed the questions; they asked subjects for both positive and negative beliefs about friendship. Asking the negative beliefs might make possible to talk about concerns related to abuse and violence.

Second, the results may be due to the fact that Cole and Bradac's (1994) subjects are different from subjects in previous research. They explained that the results perhaps differed as most of the research on beliefs about friendship was outdated, conducted over a decade ago and people's beliefs may have changed since then. This view is somewhat supported by recent research on deception in close relationships (Metts cited in Cole \& Bradac, 1994). Cole and Bradac suggested further 
investigation into this issue since previous research on beliefs about friendship did not indicate concern with abuse and violence.

Using the multidimensional scaling analysis, Cole and Bradac reported that the 43 beliefs about satisfaction with best friends are organized along three dimensions. The first dimension was interpreted as spontaneous-active vs stable-passive. The second dimension was interpreted as rational-reserved vs emotional-intimate. The last dimension was interpreted as ambitious-assertive vs easy goingunassuming. These dimensions suggest that people's beliefs about satisfaction with best friends are "primarily associated with a friend's level of activity, their emotional closeness, and their demeanor" (p. 10).

The dimensions Cole and Bradac (1994) proposed somewhat differ from the dimensions reported by previous researchers. Most previous studies have focused on the general dimensions of people's beliefs about friendships in general and have suggested dimensions such as trust, support and intimacy (e.g., Davis \& Todd, 1985; La Gaipa, 1977). Cole and Bradac specifically focused on people's beliefs about satisfaction with best friends and suggested the dimensions of a friend's level of activity, their emotional closeness, and their demeanor. Cole and Bradac suggested that people organized their beliefs about satisfaction with best friends differently from their general beliefs about friendships. In short, the study of Cole and Bradac questioned the applicability of the use of general dimensions of relationships in order to understand specific features of any relationships. 
To conclude, Cole and Bradac's (1994) study revealed that people have complex and multifaceted beliefs about satisfaction with best friends and these beliefs are organized specifically and intricately. Since their study used a sample from the U.S., the replication of the study with a Japanese sample compliments their study as well as previous friendship research and cultural research in terms of generalizability and cultural specificity of beliefs about satisfaction with best friends.

\section{Culture}

In this section, I introduce Triandis (1986) and others' (e.g., Hofstede, 1980; Triandis, Brislin \& Hui, 1991) concept of individualism-collectivism, Hall's (1976) concept of high- and low-context, and Hofstede's $(1984,1991)$ concept of uncertainty avoidance. I chose these cultural variabilities for the following reasons. One, they have often been used to explain differences between Japanese and U.S. cultures. Two, they seem most relevant to the present study of beliefs about satisfaction with best friends.

I also introduce communication behaviors that are relevant to the concepts of individualism-collectivism, high- and low-context, and uncertainty avoidance. While presenting communication behaviors, it is important to note that dimensions of cultural variability are produced as theoretical frames in order to make sense of various cultures. They do not exist separately in real life (Hecht, Andersen, \& Ribeau, 1989), but are interrelated (Hall, 1976). Therefore, it happens that some behaviors are better accounted for by more than a single dimension. It is also possible 
that different dimensions explain the same behavior. Therefore, though I discuss each dimension separately, some overlaps are found among the dimensions. In addition, these dimensions do not describe all people from a certain culture but merely explain cultural tendencies.

Hecht, Andersen, Ribeau (1989) defined culture as follows:

Culture is the manifold ways of perceiving and organizing the world that are held in common by a group of people and passed on interpersonally and intergenerationally. . . Culture has both material and symbolic manifestations, including a common code or language, heritage, history, social organization, norms, knowledge, attitudes, values, beliefs, objects, and patterns of perceptions that are accepted and expected by an identity group. (p. 163)

Hall (1992) emphasized the importance of culture on communication by stating that "culture is communication and no communication by humans can be divorced from culture" (p. 212). While previous researchers identified cultural variability dimensions (e.g., Hall, 1976; Hofstede, 1980), Ting-Toomey (1989) observed that "dimensions of cultural variability influence the underlying social structures and norms of a situation, and the social norms, in return, influence how one should or should not behave in a certain manner" (p. 352). Because culture is very important and influential to human communication, I examine the cultural variability dimensions or concepts of individualism-collectivism, high- and low-context, and uncertainty avoidance, and discuss their influences on communication both in the U.S. and Japan. 
Individualism-Collectivism

Several researchers have identified the continuum of individualism-collectivism as one of the major dimensions of cultural variation (e.g., Andersen, 1991; Hofstede, 1980; Triandis, et al., 1991). Triandis, Brislin, and Hui (1991) describe collectivism as "individuals subordinating their personal goals to the goals of some collectives." In contrast, they define individualism as "individuals subordinating the goals of collectives to their personal goals" (p. 371). In other words, individualistic cultures emphasize the importance of the self, while collectivistic cultures emphasize the importance of the group (Hofstede, 1980). Because each orientation offers a different view of the self, social group membership, and how one understands one's social world, the dimension of individualism-collectivism determines "how people live together, . . their values, and how they communicate" (Andersen, 1991, p. 289). On this continuum, the United States is considered an individualistic culture, while Japan is viewed as a collectivistic culture (e.g., Hofstede, 1980; Yum, 1991).

The concept of self or identity consists of social and personal identity (Trenholm \& Jensen, 1992). One way to understand the difference between individualistic and collectivistic cultures is to see them as differing in the balance of personal vs social self which they promote (Triandis, et al., 1991). In individualistic cultures, people value the personal self more than people in collectivistic cultures. In such cultures, the self is assumed to be "autonomous and separate from [any] groups" (Triandis, et al., 1991, p. 372). On the other hand, people in collectivistic cultures value social identity 
more than people in individualistic cultures. In such cultures, people are not just individuals; they also represent the groups to which they belong. Triandis, et al. (1991) explained the self in collectivistic cultures as follows: The self is thus defined as part of a group (e.g., family, tribe, nation) in much the same way as the body parts are part of a body. Just as it is difficult to discuss 'Jim's hand' independently of Jim, so it is unwise to discuss 'Yasumasa' independently of 'Yasumasa's ingroups.' (p. 372)

As individualistic and collectivistic cultures have different emphasis on the concept of self, they have different ideas about a group and its membership. In an individualistic culture such as the U.S., a group is viewed as merely a "collection of individuals" (Cathcart \& Cathcart, 1988, p. 186) Membership in a group is seen as a free choice which can, and should, be broken when it is no longer individually productive. Therefore, people find the group that fits their personal needs and when their needs change, they will often leave the group and join a new one. Contrarily, in a collectivistic culture such as Japan, groups are the natural "milieu in which human interaction takes place" (Cathcart \& Cathcart, 1988, p.186), and individual identity is submerged within the group. In other words, to leave a group means losing one's identity in collectivistic cultures. As a result, collectivistic cultures promote fewer groups and more intense attachments with the group.

Individualistic and collectivistic cultures also differ in the relationship of the self to a group. Because individualistic cultures emphasize the personal self, they put a high premium on independence; 
too much dependence on group affiliation is viewed as a potential weakness (succumbing to group pressure). Because individualistic cultures emphasize the autonomy of the self, they tend to promote the ideal of freedom. Collectivistic cultures, on the other hand, stress the importance of the social or group self; hence, they value interdependence more. Because collectivistic cultures promote group identity, they give great importance to the ideal of social harmony. For instance, the importance of harmony in Japan is often expressed by a well-known saying, Deru kui wa utareru (The post that sticks up is hammered).

Individualistic and collectivistic cultures differ in the way that they distribute rewards (Triandis, et al., 1991). Because each individual is conceptualized as free and autonomous in individualistic cultures, an equity norm becomes the guiding principle of social interaction. An equity norm is the idea that each member of a group is rewarded or punished according to his or her own contribution. In contrast, collectivistic cultures' emphasis on interdependence promotes the principle of equality. An equality norm is the idea that all members of a group share the same amount of rewards or punishments without much consideration of individual contribution. The principle of equality is expressed in the principle that the success of the group is the success of all individuals in the group (Cathcart \& Cathcart, 1988).

Ting-Toomey (1989) discussed individualism-collectivism by introducing the "I" identity vs the "we" identity. People in individualistic cultures tend to emphasize the "I" identity over the "we," and tend to maintain "a considerable social distance between the ' $\mathrm{I}$ ' identity and 
ingroup social influences" (p. 352). In contrast, people in collectivist cultures tend to stress the "we" identity over the "I," and tend to be "more susceptible to ingroup influences than members in the individualistic cultures" (p. 353).

Because individual attachment toward the ingroup is so strong in collectivistic cultures, individual perceptions of ingroup vs outgroup become highlighted (Triandis, et al., 1991). Since collectivists see themselves primarily as members of a group, they are "more associative within their ingroups, and more dissociative towards their out groups than are individualists" (Triandis, et al., 1991, p. 375). In other words, collectivists are more intimate with ingroup members and more formal with outgroup members than are people in individualistic cultures.

According to Nakane (1974), Japanese differentiate people into three categories within the social world based on the distinction of ingroup vs outgroup: 1) people within one's own group; 2) people whose own background is fairly well-known; and 3) people who are strangers. For example within a work setting, the first category includes co-workers in the same section or the same team. The second category may be found among all employees of the same company, or possibly those who work in the same kind of fields. The third category includes the people who are neither in the first or the second categories. Nakane (1972) posits Japanese interpersonal communication differs according to the categories people are in:

Although Japanese are quite sensitive to personal interaction, particularly with those of the first category, such a sensitivity seems 
to cease to function when facing men of the third category -- even to the extent that they may be very rude or "aggressively kind" to them. (p. 129)

Individualism and collectivism present a different view of the ideal person. While it is a common personal goal and mark of success to be independent in Western individualistic cultures, understanding the behavioral norm of the group one is in and acting accordingly is considered an attribute of the ideal person in collectivistic cultures such as Japan (Araki, 1973). Therefore, it is highly valued in Japan when one achieves his societal role by ignoring his own needs. It is also acceptable to neglect or violate the norms of an outgroup in order to achieve one's own role that is provided by the group.

\section{High-Context and Low-Context}

According to Hall and Hall (1990), a meaning is produced through the combination of an event and its context. Context is "the information that surrounds an event" (p. 6). Hall(1976) explains the difference in the way that information is transmitted in high- and low-context cultures:

A high context (HC) communication or message is one in which most of the information is already in the person, while very little is in the coded, explicit, transmitted part of the message. A low context (LC) communication is just the opposite; i.e., the mass of the information is vested in the explicit code. (p. 91)

Because low-context people "compartmentalize their personal relationship, their work, and many aspects of day-to-day life" (Hall \& Hall, 
1990, p. 7), they cannot rely on any commonly shared framework of understanding. As a result, they need detailed background information every time they interact with others. Therefore, people from low-context cultures explicitly encode more information in their communicative behaviors. In contrast, for most transactions in daily life, high-context people do not require nor expect background information to be encoded in the actual event because they assume that they already have much of the important information beforehand.

Differences in orientation can occur on an individual or a group level. However, culture is most probably the major determinant of whether someone is high- or low-context in their communication. Generally, the U.S. culture is considered to be low-context, while Japanese culture is viewed as high-context. Given that low-context and high-context cultures construct meaning differently, it is probable that even in the same situation, people from the U.S. and Japanese may appraise the same situation differently.

Low-context people tend to treat each situation separately and each person as a unique individual. In contrast, high-context people view situations and people as related one another within a given social framework. Eto (cited in Barnlund, 1989) explains this idea by contrasting Japanese with that of Westerners:

Whereas Westerners base their lives on the premise that others naturally feel differently about things and view things according to different principles, the Japanese take it for granted in their daily 
lives that other people feel and think the way as they do themselves. (p. 116)

These contrasting ideas produce very different roles for speaker and hearer within the two systems. In low-context cultures, the emphasis is on the speaker. Because people in a low-context culture do not expect that the hearer shares the same background information, the speaker's role is to make as much information as possible explicit. On the other hand, in high-context cultures, people obtain information more from the contexts rather than the actual events. As a result, they tend to have higher expectations for the hearer in interpersonal communication. Hall (1976) explains this idea as follows:

People raised in high-context systems expect more of others than do the participants in low-context systems. When talking about something that they have on their minds, a high-context individual will expect his interlocutor to know what's bothering him, so that he doesn't have to be specific. The result is that he will talk around and around the point, in effect putting all the pieces in place except the crucial one. Placing it properly - this keystone - is the role of his interlocutor. To do this for him is an insult and a violation of his individuality." (p. 113)

Because people from high-context cultures expect the hearer to understand more than people from low-context cultures do, they use a more indirect communication style. According to Searle (cited by Yum, 1991), an indirect speech act occurs, "when the speaker communicates to the hearer more than he or she actually says by referring to some mutually 
shared background information and by relying on the hearer's powers of rationality and inference" (p. 74). While most cultures have both direct and indirect communication, there is a significant difference in the level of directness which is common between low- and high-context cultures (Yum, 1991). Okabe (1987) introduces the level of Japanese indirectness when asking the hearer to shut the door as follows: instead of saying, "Please shut the door," the Japanese often say, "'It is somewhat cold today,' which is much more indirect, because no words refer to the door" (p. 134).

As a result, when people from opposite orientations interact, each may misunderstand what the other is trying to communicate. For example, people from the U.S. may complain that Japanese are long winded and "never get to the point" (Andersen, 1991, p. 293) while Japanese may complain that people from the U.S. are too direct and do not understand subtleties.

Another difference between high and low-context cultures can be found in their emphasis on verbal versus nonverbal communication. Andersen (1991) explains the perception of nonverbal communication in high-context cultures that subtleties of nonverbal behavior are "likely to be perceived by and have more meaning for people from high-context cultures" (p. 294).

While people from the U.S. think nonverbal as well as verbal communication is important, they convey or rely more on verbal code rather than nonverbal, and mainly use nonverbal code as reinforcement for the verbal. Because of this, people from low-context cultures may fail to recognize the nonverbal message that a high-context person is sending 
or not understand its subtleties. On the other hand, people from highcontext cultures may perceive more meaning than is actually meant when interpreting the behavior of someone from a low-context culture.

While nonverbal communication is less important for low-context cultures, verbal messages are highly important. On the other hand, since much information is available in the environment, it is not necessary for people from high-context cultures to state verbally the things that are obvious. Ozaki (cited in Barnlund, 1987) explains Japanese perception toward verbalization by stating "What is the worth of love if it cannot be felt without verbalizing it? The need to use words implies a lack of understanding" (p. 116). Japanese assume that the affection has been communicated nonverbally or is obvious in the circumstance, therefore do not need to or should not state verbally. For people from the U.S., on the other hand, expressing their feelings both verbally and nonverbally is important. As a result, they often make oral statements of feelings including affection such as "I love you" or "I miss you" even though the affection has been communicated in other ways.

In short, the person who is communicatively competent in the U.S. is the one who perceives every situation separately and who expresses oneself explicitly and persuasively by mainly using verbal communication. The person who communicates competently in Japan is the one who has the ability to discern the situational context and behave appropriately to the situational demands, and the ability to understand and interpret the implicit meaning in indirect or ambiguous expression (Okabe, 1983). 
High and Low in Uncertainty Avoidance

Hofstede (1984) identified four value dimensions which " affect human thinking, organizations, and institutions in predictable ways" (p. 11). They were "power distance," "uncertainty avoidance," "individualism," and "masculinity." In this section, I introduce one of these dimensions, uncertainty avoidance. Uncertainty avoidance is defined as "the extent to which the members of a culture feel threatened by uncertain or unknown situations (Hofstede, 1984, p. 113)." Japan is considered high in uncertainty avoidance whereas the United States is low in uncertainty avoidance.

According to Hofstede (1984) cultures which are high in uncertainty avoidance tend to have low tolerance in ambiguous situations, and therefore, try to avoid those situations more. Consequently, they tend to have both more rules and structures in their lives as well as listening to older people or experts more. In contrast, cultures which are low in uncertainty avoidance tend to have a high tolerance for ambiguous situations. Therefore, they have less formal rules and structures and are willing to take risks. They also possess more tolerance of people with different ideas or different backgrounds, and are generally more positive toward younger people. As a result, they tend to go abroad more and have a smaller generation gap.

Difference in the level of uncertainty avoidance relate to the difference of attitude toward the future. People in low uncertainty cultures prepare to live day by day and are more relaxed. People in high uncertainty avoidance cultures worry more about the future, have higher stress, and 
are more pessimistic in general. However, they also have higher satisfaction scores. Hofstede (1984) explains in this finding that, "if people [in high uncertainty cultures] do not consider 'leaving the organization' as a feasible alternative they will have a tendency to convince themselves that they like being in it" (p. 124).

Achievement in life differs in high and low uncertainty avoidance cultures. In high uncertainty avoidance cultures, achievement in life is "more sharply defined in terms of acquired security" (Hofstede, 1984, p. 139). In low uncertainty avoidance cultures, on the other hand, achievement in life is more related to social recognition.

The cultural dimensions of individualism-collectivism, high- and low-context, uncertainty avoidance have been introduced in this section. The dimension of individualism-collectivism differs the emphasis on the self vs the group. The dimension of high- and low-context differs in the stress of events vs contexts. The dimension of uncertainty avoidance differs in the tolerance of ambiguous situations. These dimensions are important because they may help in understanding the reasons why people's perceived beliefs about satisfaction with best friends are similar in some aspects and different in others between people from Japan and the United States. Since Japan emphasizes collectivism, high-context, and high in uncertainty avoidance, while the U.S. stresses individualism, lowcontext, and low in uncertainty avoidance, it is suspected that these cultural orientations may have influence upon people's beliefs concerning friendships, particularly best friends and those features that do/do not 
contribute a sense of satisfaction with such relationships differently between the two cultures.

In the following chapters, Japanese beliefs about satisfaction with best friends are explored. The next three chapters, chapter three through five, present an examination of Japanese beliefs about satisfaction with best friends by replicating Cole and Bradac's (1994) study. Chapter six investigates similarities and differences of underlying beliefs about satisfaction with friends between Japan and the U.S. by comparing the results of Cole and Bradac's (1994) and this studies. Chapter seven searches gender influence on beliefs concerning friendships in Japan. Finally, chapter eight concludes this study by presenting a summary of the findings, the limitations and strengths of the study and future directions. 
Chapter III

Stage One

The present study consists of five stages. This chapter describes stage one of this study. It includes purpose, methods, results, and discussion sections. In the purpose section, the purpose of this stage is described. In the method section, the sampling frame, instruments, pilot study, data collection procedure, sample characteristics, and methods of analyses are introduced. The results and discussion follow afterwards. This stage replicates Cole and Bradac's (1994) study on a lay theory of relational satisfaction with best friends by using Japanese subjects. Therefore, the discussion proceeds by comparing my results with their results.

\section{Purpose}

Purposes of this stage are to identify Japanese people's beliefs about relational satisfaction with best friends and to compare the Japanese beliefs to U.S. beliefs which Cole and Bradac (1994) reported. Thus, the following research questions are advanced:

RQ1: What do Japanese people report as beliefs about relational satisfaction with best friends?

RQ2: How similar are beliefs about satisfaction with best friends between Japan and the United States? 
It is important to identify Japanese beliefs concerning friendships because of the following three reasons. First, although previous studies identified the concept of friendships, most of these studies were conducted in the U.S. (Adams \& Bliezner, 1994). In addition, although some of the studies constructed questionnaires to examine the concept of friendship (e.g., Wright, 1985; Burleson \& Samter, 1990), most of these questionnaires were developed from a single cultural perspective, especially a cultural perspective from the U.S. Since there may be some cultural influences on people's idea about friendship, referring to the concept or questionnaire previously developed may overlook some concepts which are crucial to Japanese people's beliefs about satisfaction with friends. Therefore, this stage identifies Japanese people's beliefs about satisfaction with best friends by asking subjects to articulate their own beliefs concerning friendships.

Second, this study examines lay theories of relational satisfaction with best friends. Therefore, it is important to collect lay people's beliefs about satisfaction they have with their friends as a first step.

Third, this is a replication of Cole and Bradac's study (1994). Therefore, it is important to follow their procedure for the data collection in order to make the findings comparable to those of Cole and Bradac's. 
Methods

\section{Sampling Frame}

The sampling frame was drawn from Japanese college students in Japan. The students who fell into one of the categories below were excluded from the sample.

1. Students who previously have stayed overseas a total of more than six months.

2. Students who answered that they have best friends who are not Japanese residents.

3. Students whose age was over 25 years old. It is suspected that students who fell into categories 1 or 2 might have engaged in interpersonal intercultural relationships and that these experiences might have influenced their ideas about human relationships in a way that differs from those who have not had many intercultural experiences. The students who fell into \#3 category might be returning students and they might have different ideas about friendship because of their Social experiences as non-students.

My sample of Japanese college students does not represent all the Japanese population. However, as Nakanishi (1986) posited:

the young generation is an important segment of any society and may be more sensitive to the contemporary values of the culture than the older generation. In addition, the use of the college-age population increases the comparability of the results to studies 
conducted in the United States that are largely based on a similar type of sample. (p. 173)

Cole and Bradac (1994) drew their sample from college students in the United States. In order to make findings in this stage comparable to those of Cole and Bradac's study (1994), it seems most appropriate to draw a comparative sample from Japanese college students in Japan.

\section{Instruments}

In order to identify Japanese people's beliefs about satisfaction with best friends, a questionnaire was constructed. As this study was replication of Cole and Bradac (1994), I referred to their questionnaire. Cole and Bradac's (1994) questionnaire consists of instructions and two open-ended questions. In order to make the Japanese questionnaire, I did not use the direct translation of their questionnaire because direct translation did not sound natural and for replication it seemed more important to deliver the same content rather than being overconcerned with a translation of each word. Two Japanese graduate students in the U.S. who are fluent in both Japanese and English in the U.S. checked my questionnaire in order to examine whether the content was the same as a questionnaire of Cole and Bradac (1994), and some changes were made.

Four fill-in-blank type of questions were added to the Japanese questionnaire. These questions were taken from Kono, Maeda, Nishishiba, and Peterson's (1995) study on the American and Japanese concepts of friendship. I added these questions because I thought there might be some students who would not be able to answer open-ended questions, and fill- 
in-the-blank questions might help them come up with ideas about their best friendship.

The questionnaire consisted of four pages. On the first page, subjects were asked to indicate all the characteristics and qualities they thought a best friend should possess in order to create a satisfying relationship (Question \#1). Subjects were provided with fifteen blank spaces in which to respond.

On the second page, the subjects were asked to indicate all the characteristics and qualities they would think lead to dissatisfaction in this type of relationship (Question \#2). Again, fifteen blank spaces were provided.

On the third page, the subjects were asked to fill in the blanks to complete the sentences (Question \#3). There were four sentences:

1. Best friends should be... (character, personality, quality)

2. Best friends should not be... (character, personality, quality)

3. Best friends should ... (behavior, verb, action)

4. Best friends should not ... (behavior, verb, action) The subjects were provided five blank spaces for each sentence.

On the forth page, subjects were asked to provide their personal information (see Appendix D). The information includes subject's age, sex, the length and places of living overseas, and whether she/he has best friends who are not Japanese residents. Some of these questions were used to exclude subjects who do not meet the sample framework of this study. In addition, subjects were asked for information about the people they considered as their best friends. This included the number of their best 
friends, and age and sex of each best friend, as well as the way the subjects and their best friends became acquainted. This information is important because it provides insight into who Japanese people consider to be their best friends.

\section{Pilot Study}

A pilot study was conducted to examine whether the questionnaire was easy to answer for the target subjects, whether it would include fill-in the blank questions, and whether subjects could complete the questionnaire within thirty minutes.

Five Japanese college exchange students who had been in the United States less than six months participated in the pilot study. They did not have any difficulty answering the open-ended questions as well as fill-inthe-blank questions. although some of their answers for fill-in-the-blank questions were the same as their answers for open-ended questions, some answers appeared only in the fill-in-the-blank questions. Therefore, fill-inthe-blank questions were kept in a questionnaire in order to gather as many ideas as possible about relational satisfaction with best friends. all students completed a questionnaire within the expected time frame.

The students who participated in the pilot study gave their thoughts about the questionnaire after they completed it. Although some students expressed some difficulties producing responses, the numbers of their responses (average of 30 per person) showed their capability of doing the task. Therefore, the question format stayed the same. Some students commented that when they filled in the blank in Question \#3, the word 
did not fit in the sentence. In order to avoid hesitation toward making an unnatural sentence, an example was added. The example explained that

"A best friend should do " can be used "A best friend should do write " even though usual sentence should be "A best friend should write." Through the comments of the students who participated in the pilot study, some other minor changes were made in order to make it easier for the target subjects to fill in the questionnaire.

\section{Data Collection Procedure}

In May 1995, I contacted four college instructors, explaining the purpose of this research, and asked for cooperation for data collection from their students. After receiving their permission, I mailed them informed consent forms, questionnaires, and instructions about how to conduct this questionnaire (see Appendixes A, B, \& C).

The same procedure was followed by each instructor. The questionnaires were distributed to the students by their instructor during a class period. Students were informed of the purpose of the study and asked to sign two informed consent forms if they agreed to participate in the study. One consent form was submitted to the instructor and the other form was kept by each student. Then, the instructor gave the instructions for the 4 page questionnaire (see Appendix $C$ for the detail) and distributed it to each student. Students were given thirty minutes to complete it. After completing the questionnaire, they returned it to the instructor. I received all questionnaires and informed consent forms from the four instructors by mail in the United States in July 1995. 
Sample characteristics

A total of 167 Japanese students from four colleges filled in a questionnaire (74 females, 93 males). Of these students, 19 did not match the sampling frame of the study. Three students did not answer any of the questions. Therefore, the sample of stage one consisted of 145 Japanese graduate and undergraduate students (69 females, 76 males).

\section{Data analysis}

Data included descriptions concerning relational satisfaction with best friends. In order to identify Japanese people's beliefs about relational satisfaction with best friends, data were analyzed qualitatively. While concepts should emerge from the data and all concepts should be identified as a typology of satisfaction with best friends, I targeted the number of concepts between 30 and 45 for two practical reasons:

1) comparability to Cole and Bradac's (1994) 43 concepts (see Appendix E for their concepts).

2) manageable size of concepts in order to conduct the card-sorting task used in stage two.

From the 145 questionnaires, two thirds of them (97 questionnaires; 46 females, 51 males) were randomly selected in order to create an initial list of beliefs about satisfaction with best friends. The remaining one third of questionnaires was kept to check the categories which emerged from the initial list.

The responses of the 97 questionnaires were combined and a list of over 1,350 descriptions was constructed. This list includes both negative 
and positive responses. According to Cole and Bradac (1994), an initial list should include both the positive and negative responses in order to "obtain a wide range of beliefs" (p. 6) about a best friend. I agreed with their decision because in many cases, the descriptions used to describe their dissatisfaction with best friends explain people's boundary or bottom line of satisfaction with best friends. For instance, many subjects answered that a best friend "being selfish" prompted their dissatisfaction in Question \#2 but not many subjects answered that a best friend should possess the quality of "not being selfish" in order to create a satisfying relationship in Question \#1. In this case, a best friend "not being selfish" is an important element in a satisfying relationship with a best friend. However, this answer is hard to elicit when asked in a positive manner because a best friend not being selfish is a basic assumption for many people.

In order to treat all responses in a same manner, "not" was added to all negative responses that emerged from question \#2, \#3-2 and \#3-4 (see Appendix A) and those were treated as positive responses. For, instance, a description of "being selfish" as an answer of \#2 was changed to "not being selfish." This task made all the responses as descriptions of relational satisfaction with best friends.

From the list, identical descriptions and obvious redundancies of the descriptions were discarded. For instance, the descriptions of "keep his/her word" and "does not break his/her promise" were considered as obviously redundant. Therefore, one of them were discarded. This task reduced the data and created the initial list of 211 descriptions. 
Cole and Bradac (1994) removed idiosyncratic items because they assumed that extreme idiosyncratic items such as "likes soap operas" and "doesn't listen to cheesy dance music all the time" are "very specific representations of the frequently mentioned belief that a best friend should share similarities " (p. 6). There were some idiosyncratic descriptions such as "likes sports," "drinks sake together," and "a company who worked hard together aiming the same goal in a club or something" in the initial list. However, I was not sure what concept these specific descriptions represent. Therefore, these descriptions remained in the initial list.

Each of the 211 descriptions related to Japanese beliefs about satisfaction with best friends was written on a card. Three Japanese people who were familiar with this study first examined all 211 descriptions, then sorted these cards into logical groupings. As criteria for identifying groups, Cole and Bradac (1994) grouped the items when "there was an agreement that the items shared a common meaning" and when "each grouping represented more than a single student's response" (p. 6). This study followed their criteria.

When grouping the cards, one person chose one card to group with other card or cards. The grouping was accepted when the other two members agreed with the decision. This process was done by the three people taking a turn in a round robin style in order to give each person an equal chance to group the items. After grouping all the items, the three people named the groupings together. For example, descriptions such as "reliable," "does not let me down," and "a person who I can rely on" were 
grouped together and named as "trustworthy." This task sorted 211 descriptions into 43 categories.

In order to define the typology of best friendship carefully, five steps were followed. First, within one third of the questionnaires which were not used, a half of them ( 24 questionnaire, 11 females, 13 males) were randomly selected. Two Japanese people examined the descriptions in these 24 questionnaires, for whether these descriptions would fit into the 43 original categories. While most descriptions fit into the original categories, some descriptions did not fit adequately. Therefore, one new category called "Encourages me" ("Hagemashite kureru") was added and 44 categories were identified.

Second, two groups of Japanese people sorted the initial list of 211 descriptions into groups without using the previously identified categories and named the groupings. When both of the two groups identified the same category and this category was the same as one of the 44 categories previously identified, this category remained in the final list of categorization.

Third, one sixth of the questionnaires (24 questionnaires, 12 females, 12 males), not previously used, provided 155 descriptions after discarding identical descriptions and obvious redundancies. Three Japanese people who were familiar with this study received a set of 155 cards on which the descriptions were written. They individually grouped these cards without using already identified categories and named the groupings. If two of the three people identified the same category as one of the 44 categories, this 
category remained in the final list of categorization. Through out of second and third steps, 31 categories remained in the final list.

In the fourth step, when the categories were identified by one of the groups in the second step and one of the persons in the third step, these categories remained in the final list. Five categories remained in the final list through this step. They were "positive" ("Maemuki de aru"), "can confide in" ("Kuchi ga katai"), "does not look down on me" ("Watashi wo mikudasanai"), "a person I spend long time together" ("Tomoni sugoshita jikan ga nagai"), and "does not brag about him/herself" ("Jiman wo shinai").

The fifth step examined each of the eight categories which were identified in the first step but did not match the criteria of the second, third and fourth steps. If the theme of the eight categories was represented by the other 36 categories already on the final list, they were discarded from the final list. If they were unique themes not represented by any of the 36 identified categories, they remained on the final list. This examination retained three categories and discarded five categories from the final list. The categories which remained after this process were "sensible" ("shiryo bukai"), "listens to a partner's opinion" ("aite no iken wo kiku"), and "give and take relationship" ("mochitsu motaretsu no kankei de aru"). The categories which did not remain were "not mean" ("ijiwaru de nai"), "a special existence" ("tokubetsu na sonzai de aru"), "does not impose his/her opinion" ("jibun no iken wo oshitsuke nai"), "not shy of me" ("enryo shinai"), "a relationship that keeps a certain distance" ("ippo hikaete sessuru"). Consequently, the final list consisted of 39 categories. 
After 39 final categories were determined, each category name was re-examined by three Japanese people to see whether it best described all the items of the category. They examined all the descriptions in each category to see whether one of these descriptions clearly represented its category. If it did, this description was chosen as a category name. If not, a category name which seems to represent the grouping best was created. Category names which emerged from the subject's description were chosen if possible, because these category names would be used in stage two for subjects in the same sample framework as stage one. I assumed that it would help the subjects in stage two to capture the meaning of the category clearly if the category names were familiar to those subjects. Fifteen category names were either changed or shortened through this examination. The 39 categories were identified as a typology of perceived beliefs about relational satisfaction with best friends (see Table 3-1). This number satisfied a target number of between 30 and 45 which is discussed in page seven.

Results

The 39 categories were identified as a typology of Japanese people's perceived beliefs about relational satisfaction with best friends. They include both positive and negative responses. They include best friend's characteristics (e.g., calm, big-hearted), attitudes (e.g., listens to a partner's opinion, advises me), and characteristics of the relationship (e.g., a relationship that feels at ease, a give-and-take relationship). 
Table 3-1.

\section{Lapanese Perceived Beliefs of Relational Satisfaction with Best Friends}

1. Does not depend on a friend too much (Aite ni izon shisuginai ): can take care of him/herself, does not depend on others too much

2. Listens to a partner's opinion (Aite no iken wo kiku): listens to my story, does not force his/her opinion

3. A rival in a good sense (Ii imi deno raibaru dearu): a good rival, we stimulate each other, stimulative

4. Joyful to be with (Issyoni ite tanoshii): we enjoy together, a happy person, a cheerful person, fun

5. Does not lie (Uso wo tsukanai): does not keep things back, honest, faithful

6. Considerate (Omoiyari ga aru): can understand how other people feel, kind, has consideration, does not do spiteful things to others

7. Says what he/she thinks clearly (Omotteiru koto wo hakkiri iu): tells what he/she thinks, says things clearly

8. Calm (Onkou de aru): not short-tempered, gentle

9. Has similar values (Kachikan ga niteiru): has similar ideas, has the same value

10. A person I get along well with (Ki ga au): we are on the same wave-length, we have similar sensitivity, we have the same sense of humor

11. Relationship that feels at ease (Ki wo tsukawanai kankei de aru): frank, I can relax when being with him/her, we can spend time without conversation

12. Can confide in (Kuchi ga katai): holds a secret, does not talk to other people what I consulted to him/her

13. Big-hearted (Kokoro ga hiroi): tolerant, does not worry about details

14. Not selfish (Jibun katte de nai): cooperative, not self-centered, not egoistic

15. Has a sense of self (Jibun to iumono wo motteiru): has his/her own idea, has his/her own belief, not a follower, not indecisive

16. Does not brag about him/herself (Jiman wo shinai): does not always brag about him/herself, does not behave self-important

17. Has common sense (Joushiki ga aru): behaves according to circumstances, has good social manners, does not do things against morals

18. Sensible (Shiryo bukai): cool, not too excitable, does not behave hastily

19. Caring (Shinmi ni natte kureru): concernd for me, thinks about me seriously

20. Trustworthy (Shinrai dekiru): reliable, a person who I can rely on, does not let me down

21. Straightforward/Not hypocritical(Sunao de aru): does not have a split/double face. apologizes when he/she is wrong 
22. Has a sense of responsibility (Sekininkan ga aru): has a strong sense of responsibility, not irresponsible, keeps his/her promise

23. Treats everyone equal(Dareni demo byoudou de aru): does not change his/her attitude depending on people, does not judge people according to their past

24. Advises me (Cyuukoku shite kureru): tells me when I am wrong, sometimes stern, points out my mistakes

25. Sociable (Tsukiai ga ii): can play with me when I want

26. A person I spend a long time with (Tomoni sugoshita jikan ga nagai): spends long time with me, a companion who worked hard together with me aiming towards the same goal in a club or something

27. A person I can have a long-term relationship with (Nagai tsukiai ga dekiru): we can keep a relationship even though we live apart, we get along for a life time

28. A person I can consult about anything (Nandemo soudan dekiru): I can talk about anything, a relationship that can have consultation

29. Encourages me (Hagemashite kureru): encourages me when I am down, cheers me up

30. Deals with a problem seriously when needed (Hitsuyounatoki ni majime ni taiou shite kureru): can be serious when having a serious conversation, does not make fun of me when I have a serious talk, can think about things seriously

31. Does not speak ill of others (Hito no waruguchi wo iwanai): does not talk behind people's back

32. Positive (Maemuki de aru): active, a person who perseveres, a person who desires to improve $\mathrm{him} /$ herself, a person who has a positive attitude

33. A give-and-take relationship (Mochitsu motaretsu no kankei de aru): we meet each other halfway, we help each other, we give and take

34. Does not pry too much (Yokei na koto wo sensaku sinai): does not interfere in others' affairs too much, does not ask too much of a private life, we keep a certain distance each other

35. A relationship in which we keep in contact with each other (Renraku shiau): we contact periodically, we write or call

36. Has something I can learn (Watashi ga manaberu nanika wo motteiru): has something I do not have, a person I can respect, has a lot of knowledge

37. Has similarities with me (Watashi to kyoutsuushita ten ga aru): has the same interests or hobbies, has the same ability and economic strength as me

38. Understands me (Watashi no koto wo rikai shiteiru): knows my personality, accepts who I am

39. Does not look down on me (Watashi wo mikudasanai): does not make fun of me, does not say terrible things about me 
Discussion

Thirty-nine categories of relational satisfaction with best friends were identified from the data collected from Japanese college students. These categories were compared with Cole and Bradac's (1994) 43 categories of relational satisfaction with best friends which emerged from the U.S. subjects (see Appendix E for Cole and Bradac's categories). Although the categories for Japanese subjects were identified without using Cole and Bradac's (1994) categories, there are similar categories between the two groups (see Table 3-2). Many of these categories are supported by previous friendship researchers. For instance, Davis and Todd (1985) mentioned the importance of enjoyment, understanding for friendship and trust (see page 18 for more detail). The quality of enjoyment is expressed as "joyful to be with" (Japan) and "fun to be around" (the U.S.). The importance of understanding for friendship is demonstrated as "understands me" (Japan) and "intimate" (the U.S.). The idea of trusting is presented as "trustworthy" (Japan) and "dependable" (the U.S.).

La Gaipa (1977) reported the concepts of helping behavior and similarity as important in friendship. Helping behavior for Japanese subjects was expressed as "advises me" while this behavior for U.S. subjects was presented as "inspiring." although these two concepts do not share the same behaviors, each of these behaviors seems to illustrate a quality of help in each culture. The concept of similarity is demonstrated as "has similarities with me," "has similar values," and "a person I get along well with" (Japan) and "share similarities" (the U.S.). 
Table 3-2

Similar categories of relational satisfaction with best friends:

\section{Lapan and the United States}

\section{Japanese Categories}

\section{U.S. Categories}

Concept of enjoyment suggested by Davis and Todd (1985)

4. Joyful to be with (Issyoni ite tanoshii): we enjoy together, a happy person, a cheerful person, fun
22. Fun to be Around: Has a great sense of humor and is always fun to be with. Very entertaining and likes to joke around.

concept of understanding suggested by Davis and Todd (1985)

38. Understands me (Watashi no koto wo rikai 30 . Intimate: Shares their secrets with me. Knows shiteiru): knows my personality, accepts who I am me well and I also know their innermost ideas.

concept of trust suggested by Davis and Todd (1985)

20. Trustworthy (Shinrai dekiru): reliable, a person who I can rely on, does not let me down

15. Dependable: Keeps his word. Is trustworthy, punctual, and doesn't break promises.

concept of helping behavior suggested by La Gaipa (1977)

24. Advises me (Cyuukoku shite kureru): tells me when I am wrong, sometimes stern, points out my mistakes
28. Inspiring: Is my role model. Inspires me to be a better person, gives me advice, and makes me think about my ideas.

concept of similarity suggested by La Gaipa (1977)

9. Has similar values (Kachikan ga niteiru): has similar ideas, has the same value

10. A person I get along well with (Ki ga au): we are on the same wave-length, we have similar sensitivity, we have the same sense of humor 37. Has similarities with me (Watashi to kyoutsuushita ten ga aru): has the same interests or hobbies, has the same ability and economic strength as me

43. Share Similarities: Has many things in common with me. Has similar values, morals, interests, career goals, and political views. Shares my sense of humor and likes to do the same things that I do.

concept of duration suggested by Young (1986) and Synder and Smith (1986)

26. A person I spend a long time with (Tomoni sugoshita jikan ga nagai): spends long time with me, a companion who worked hard together with me aiming towards the same goal in a club or something

27. A person I can have a long-term relationship with (Nagai tsukiai ga dekiru): we can keep a relationship even though we live apart, we get along for a life time
9. Available: Has plenty of time to spend with me and is always willing to make time for me. 11. Committed: Acknowledges our friendship; is willing to make me a part of their life. Interested in a long term involvement with me. 
A concept of duration was reported as an important component of close friendship by Young (1986) and Synder and Smith (1986). This component is found as "a person I spend a long time with" and " a person I can have a long-term relationship with" from Japanese categories and "available" and " committed" from the U.S. categories.

In short, the similar categories found in Japanese and U.S. data indicated that there are shared concepts about relational satisfaction with best friends between the two cultures. They are the concepts of enjoyment, understanding, trust, helping behavior, similarity, and duration.

There are differences in categories of satisfaction between Japanese and U.S. data (see Table 3-3). In the U.S. categories, there are three concepts which seem to relate to stimulus: "active/energetic," "activities director," and "creative." These categories suggest that U.S. subjects want best friends to be adventurous, creative, unique, and risk takers.

However, there seems no Japanese categories related to stimulus. Instead of looking for a stimulating quality from best friends, Japanese subjects seek a comfortable relationship for their best friends: a relationship in which they can relax or one where they spend time together without talking ("relationship that feels at ease"). The equivalent idea is not found from the U.S. categories. 
Table 3-3

\section{Different categories of relational satisfaction with best friends}

Only in the Japanese Categories

High in Uncertainty Avoidance 11. Relationship that feels at ease (Ki wo tsukawanai kankei de aru) : frank, I can relax when being with him/her, we can spend time without conversation
Only in the U.S. Categories

Low in Uncertainty Avoidance

2. Active/Energetic: The active adventurous type. Is bold, daring, and risk taker.

3. Activities Director: Comes up with creative activities, introduces me to others, and always invites me to do things.

14. Creative: Is a little off beat, unique, and holds interesting ideas.

\section{Collectivism}

12. Can confide in (Kuchi ga katai): holds a secret, does not talk to other people what I consulted to him/her

20. Trustworthy (Shinrai dekiru): reliable, a person who I can rely on, does not let me down 22. Has a sense of responsibility (Sekininkan ga aru): has a strong sense of responsibility, not irresponsible, keeps his/her promise

9. Has similar values (Kachikan ga niteiru): has similar ideas, has the same value 10. A person I get along well with (Ki ga au): we are on the same wave-length, we have similar sensitivity, we have the same sense of humor 37. Has similarities with me (Watashi to kyoutsuushita ten ga aru): has the same interests or hobbies, has the same ability and economic strength as me

29. Encourages me (Hagemashite kureru): encourages me when I feel down, cheers me up

\section{Individualism}

15. Dependable: Keeps their word. Is trustworthy, punctual, and doesn't break promises.
43. Share Similarities: Has many things in common with me. Has similar values, morals, interests, career goals, and political views. Shares my sense of humor and likes to do the same things that I do.
32. Not a Whiner: Doesn't dump their problems on me. Able to handle their own problems and stress and doesn't complain to me.

20. Family Oriented: Has traditional family values, a good family background, and is family oriented.

25. Healthy: Is athletic. Takes care of their body and doesn't abuse alcohol and drugs.

34. Not Physically Violent: Is not a violent person.

Those differences can be explained by Hofstede's $(1984,1991)$ cultural dimension of uncertainty avoidance. According to Hofstede (1991), uncertainty avoidance can be defined as "the extent to which the members of a culture feel threatened by uncertain or unknown situations (p. 113)." As a result, people from a culture which is high in uncertainty avoidance 
tend to prefer structure or pattern and avoid unexpectedness in a daily life including relationships (Hofstede, 1991). Japan is considered high in uncertainty avoidance whereas the United States is low in uncertainty avoidance. The U. S. subjects looking for adventurous and creative best friends indicate that they not only accept but also enjoy unpredictability in their best friends. In contrast, Japanese subjects may have lower tolerance about unpredictable behavior of best friends because they feel threatened rather than excited, and therefore do not necessarily look for adventurous or creative best friends. Instead, they seek predictable and comfortable relationships with their best friends. These findings are compatible with Peterson et al. 's (1994) study on the American and Japanese concepts of friendship. In their study, the tendency for "newness and changes" was found only in the U. S. subjects.

In the Japanese data, there is a category called "a rival in a good sense," which describes best friends as stimulative. However, this category seems to indicate stimulative by competing with each other. People usually compete with those who are not too different. In this sense, this category may hold a basic assumption that best friends are similar. In addition, this category does not necessarily require best friends to be adventurous or creative. Therefore, the categories of "active/energetic," "activities director," and "creative" seem unique to the U.S. culture when compared to the Japanese culture.

The category "encourage me" from Japan and "not a whiner" from the U.S. is an interesting contrast between the two cultures. This difference can be explained by the cultural dimension of collectivism-individualism. 
Japan is considered a collectivistic culture whereas the U.S. is considered an individualistic culture (e.g., Hofstede, 1980; Triandis et al , 1991).

Collectivistic cultures such as Japan stress the importance of the social or group self; they value interdependence more than individualistic cultures (Hofstede, 1980). Therefore, it is understandable that Japanese people not only expect to share their problems with their friends; they also expect those friends to encourage them to overcome the problems. The relationship that provides space for these behaviors can be seen as a representation of a highly developed friendship. If their best friends do not cheer them up, these friends may be considered irresponsible in Japan. As a result, people may perceive that their relationship is strong enough to take care of one another in Japan.

Individualistic cultures such as the U.S., on the other hand, emphasize the personal self; they highly value independence. In the U.S., although friends help each other, too much dependence on others can be viewed as personal weakness rather than the strength of a relationship. Therefore, the U.S, subjects may want their best friends not to be a whiner but to be independent enough to take care of themselves.

Although similar concepts were found in the two cultures, there are two concepts in which that the number of categories related to these concepts differs between the Japanese and the U.S. data. Whereas in the U.S. there is a category "dependable," there are three categories which seem to relate to dependability in the Japanese data: "can confide in," "trustworthy," and "has a sense of responsibility." In addition, in the U.S. category "share similarities," there are three Japanese categories which 
seem to illustrate similarity: "has similar values," "a person I get along well with," and "has similarities with me." There are two possible explanations. One is that these differences were merely due to the differences of the way coders in Cole and Bradac's (1994) study and this study grouped the words rather than differences of subjects' descriptions about relational satisfaction with best friends.

Two is that these differences were due to the differences in detail of subjects' description about these two concepts. In other words, three categories concerning dependability as well as similarity emerged from the Japanese data because Japanese data contained many detailed descriptions related to these concepts and this variety of descriptions produced three categories concerning dependability and three categories concerning similarity. For Japanese subjects, these concepts may have greater weight within beliefs about relational satisfaction with best friends. In turn, they may have come up with more elaborate concepts concerning dependability and similarity than other concepts. If so, the idea of Japanese valuing dependability and similarity of their best friends is consistent with the Japanese cultural tendency of collectivism.

People in collectivistic cultures such as Japan are more associative within their groups, and more dissociative towards their outgroups than people in individualistic cultures (Triandis, et al., 1991). Within their groups such as a family or a circle of friends, members depend on each other. Furthermore, because best friends are the core members of a circle of friends, people may expect their best friends to be dependable in every way. Their expectations about best friends may be as high as their expectations 
about themselves. Therefore, the reason that there were many categories related to dependability in Japanese data may be the result of the reflection of this higher expectation toward best friends.

The reason that there are three categories of related similarities may also be explained by Japanese collectivistic cultural tendency. People in collectivistic cultures value harmony (Triandis, et al., 1991). One way to maintain harmony or avoid conflicts within the in-group is to increase similarities among members. If members in the in-group have the same values or same interests, there is less conflict, and in turns, harmony can be easily maintained. In order to maintain their circle of friends, Japanese subjects may have valued similarities with their best friends strongly.

The categories of "family oriented," "healthy," and "not physically violent" appeared only in the U.S. data. They may reflect societal problems such as divorce, drug usage, and physical abuse in the United States. The subjects in Cole and Bradac's (1994) study might be more sensitive about these issues and also aware of how crucial these issues are to human relationship. Therefore, they might desire that their best friends possess strong family values, practice only moderate use of alcohol or drugs and that they are non-violent in conflicts with others. Although all of these issues are in people's interest in Japan as well, my subjects, Japanese college students in Japan, may not associate these issues as important when being asked about relational satisfaction with best friends.

Another interpretation is that these three categories may indicate that people are looking for friends from a similar social level or orientation For instance, people who have good family values may desire 
their best friends to have the same values. Since the U.S. is a country in which diverse cultures exist, people may feel a need to define values so as to attract similar friends. Japan, on the other hand, is a rather homogeneous culture. In such a culture, people may assume that others have similar family values. Therefore, they may not need to state what values they want their friend's to possess.

However, as Cole and Bradac (1994) mentioned, since none of the previous researchers found concepts of abuse and violence concerning friendship, these results need to be treated cautiously. Their subjects are college students who are considered to be highly educated middle class younger people. These specific conditions may have some influence on the results. Therefore, it is unknown whether these concepts are issues for other U.S. people when they think of relational satisfaction with best friends.

\section{Conclusion}

In this stage, Japanese beliefs of relational satisfaction with best friends were identified. They include many variety of descriptions such as best friend's characteristics and attitudes, and the characteristics of relationship. Japanese people's beliefs were compared with Cole and Bradac's (1994) U.S. people's beliefs. As a result, similarities as well as differences between the two cultures related to people's beliefs about relational satisfaction with best friends were revealed. 
Similar categories found between the two cultural groups are concepts of enjoyment, understanding, trust, helping behavior, similarity, and duration. These concepts are supported by previous friendship researchers. Differences which emerged from Japanese and the U.S. data were as follows:

1) Japanese look for a comfortable, predictable relationship rather than a stimulating relationship, while U.S. subjects look for a stimulating friendship rather than a stable relationship.

2) Japanese tend to value interdependent characteristics, specifically in-group responsibility over independent characteristic, while U.S. subjects value independent characteristic over interdependent characteristic.

These differences were explained by cultural tendencies of (a) uncertaintyavoidance and (b) individualism-collectivism. Within above cultural tendencies, Japanese and the U.S. subjects tend to emphasize opposite dimensions. Japan is a collectivistic culture and high in uncertaintyavoidance, whereas the U.S. is an individualistic culture and low in uncertainty-avoidance.

This stage revealed that people from Japan and the U.S. hold some similar beliefs concerning relational satisfaction with best friends. This finding suggests that people from different cultural backgrounds can become good friends because they share similar concepts related relational satisfaction with best friends. However, at the same time, some differences in beliefs about satisfaction with best friends were found. This finding suggests that people from Japan and the U.S. may have different behaviors 
as well as expectations about relationship with best friends. In turn, these differences may become obstacles between people from Japan and the U.S. trying to develop a friendship. 
Chapter IV

Stage Two

This chapter describes stage two of the present research. The chapter consists of four sections: purpose, methods, results, and discussion sections. In the purpose section, the purpose of stage two is presented. In the method section, the sampling frame, instruments, data collection procedure, sample characteristics, and method of analyses are described. In the results section, determination and interpretation of dimensional solution are introduced. The discussion section follows afterwards.

\section{Purpose}

This stage explores how Japanese people cognitively organize their beliefs about relational satisfaction with best friends and whether these beliefs are organized in particular, meaningful ways. In order to answer these questions, I examines the way subjects perceived the relationships among words which were identified as beliefs about satisfaction with best friends in stage one.

These perceived relationships may reveal people's underlying structure of beliefs about satisfaction among best friends, if any. It is important to examine the underlying structure of these beliefs, because it is assumed that people use this structure in order to understand the 
behaviors and traits of their best friends. Therefore, the following research question is advanced:

RQ3: How do Japanese people organize the beliefs about relational satisfaction with best friends in their mind?

\section{Method}

\section{Sampling Frame}

The sampling frame was drawn from Japanese college students in Japan. The students who fell into one of the categories below were excluded from the sample.

1. Students who previously have stayed overseas a total of more than six months.

2. Students who answered that they have best friends who are not Japanese residents.

3. Students whose age was over 25 years old.

The reasons for above exclusion are the same as stage one which are explained in page 43 .

\section{Instruments}

In order to examine how Japanese people cognitively organize their beliefs about satisfaction with best friends, a card sorting technique was administered. The procedure of this technique was as follows:

Subjects received a set of 39 cards and a questionnaire (see Appendix B \& F). On the cards, 39 beliefs of Japanese people toward relational 
satisfaction with best friends (identified in stage one) were printed. Subjects were instructed to read all the words on the index cards and arrange the cards into different groups based on their perceived similarities. Subjects were told that they could make as many groups as they like, that they could form a group from one card if they want, and that they could rearrange their groupings. Each card had a unique number. When subjects finished the task, they recorded their results by listing the card numbers according to their groups on an answer sheet provided.

Subjects were then directed to the next question which is presented in chapter seven. Lastly, the subjects were asked personal information as well as the information about the people they considered to be their best friends (see Appendix D for more detail). This questionnaire format was exactly the same as the one which was used in stage one.

\section{Data Collection Procedure}

I contacted three college instructors in Japan, explaining the purpose of this research and asked for their cooperation for data collection. I received their permission to use 30 minutes of class time for the data collection, and all data were collected in October 1995.

The same procedure was followed in each class. An instructor introduced me to the students, and I informed them of the purpose of this study. Then, students were each provided a questionnaire with an informed consent form and a set of 39 index cards on which Japanese people's beliefs about satisfaction with best friends were printed (see Appendix B and F). 
The first page of the questionnaire was an informed consent form. If students agreed to participate in this study, they were instructed to go on to the following page. After completing the questionnaire, they returned it to me. I visited six classes at three colleges for this stage and collected 352 questionnaires.

\section{Sample characteristics}

A total of 352 Japanese undergraduate students from three colleges engaged in the card sorting task (193 females, 159 males). Of these students, 52 did not match the sampling frame of the study or had missing data. Thirty-five students either did not use all of the 39 words or used the same word more than once. Since the program I used to run statistical analyses required a subject to use all the words once, these data were discarded from the sample. Therefore, the sample of stage two consisted of 265 Japanese undergraduate students (143 females, 122 males).

\section{Method of analyses}

In order to reveal Japanese people's underlying structure of beliefs about relational satisfaction with best friends, multidimensional scaling and hierarchical cluster analysis were conducted using SPSS $® 6.1$ software. Kruskal and Wish (1978) explained multidimensional scaling as a class of techniques. These techniques:

use proximities among any kind of objects as input. A proximity is a number which indicates how similar or how different two objects are, or are perceived to be, or any measure of this kind. The chief 
output is a special representation, consisting of a geometric configuration of points, as on a map. Each point in the configuration corresponds to one of the objects. This configuration reflects the "hidden structure" in the data, and often makes the data much easier to comprehend. By reflecting the data structure we mean that the larger the dissimilarity (or the smaller the similar) between the two objects, as shown by their proximity value, the further apart they should be in the spatial map. (p. 7)

In order to conduct multidimensional scaling (MDS) for this stage, the results of card-sorting were converted into a co-occurrence matrix. Each subject sorted the 39 words into groups. Each grouping consisted of words which a subject thought were similar. Since the words in the same group were perceived as similar, the proximity of any combination of pairs in the same group was coded as zero, while the proximity of any other combination of pairs was coded as one.

All pairs of the 39 words were compared by above criteria, and a dissimilarity matrix of $39 \times 39$ with zero and one was constructed for each subject. A dissimilarity matrix of $39 \times 39$ for all the subjects was constructed by aggregating all the individual matrixes. The smaller the number is in the matrix, the more the subjects perceived the pair of words as similar. This matrix presented subjects' perceived similarities/dissimilarities of 39 beliefs about relational satisfaction with best friends.

By using the aggregated dissimilarity matrix, MDS performs a spatial map by plotting the words closer to each other when the number in the matrix is smaller. In this sense, a number in the matrix is perceived as a 
distance. Solutions were calculated using one to six dimensions. The dimensional solution for Japanese beliefs about relational satisfaction with best friends was determined by examining both the stress and interpretability of a map.

In MDS, stress is a measure of how well a map represents all the distances in the matrix. While stress usually decreases when dimensions increase, substantial decrease of stress by adding an extra dimension is one of the keys to determine dimensionality. When plotting the stress along the number of dimensions, the line creates an elbow-like curve if there is a dramatic drop of stress. The dimensionality in which this elbow appears may be the appropriate dimensionality. However, a dimensional solution that provides stress much above 0.10 is not recommended (Kruskal \& Wish, 1978) because, if stress is high, a map does not accurately reflect the matrix and, in turn, does not properly reflect card sorting results.

Interpretability is determined by visual inspection of the position of all the words along each dimension of a map. According to Kruskal and Wishes (1978), "interpretability often plays a central role in choosing the particular dimensionality within the range of reasonable dimensionalities" (p. 57) which are suggested by stress. When conducting a visual inspection, the hierarchical cluster analysis assists the interpretation of a dimension or dimensions.

According to Hair, Anderson, Tatham, and Grablowsky (1979), hierarchical cluster analysis is described as "an analytical technique which can be used to develop meaningful subgroups of individuals or objects" (p. 8). The groups are not pre-determined, but "the technique is used to 
identify the groups" (p. 8). Hierarchical cluster analysis was conducted by using the same dissimilarity matrix used for MDS analysis.

Since "clusters are formed by grouping cases into bigger and bigger clusters until all cases are members of a single cluster" (Norusis, 1994, p. 85) in hierarchical clustering, a reasonable number of cluster solutions must be determined. In order to find the cut-off point for cluster solution, major jumps in the coefficient of "Agglomeration Schedule using Single Linkage" were investigated. A stage before "the (coefficient) increase between two adjacent steps becomes large" (Norusis, 1994, p. 91) is considered the best solution. The subgroups, which were defined by hierarchical cluster analysis, were superimposed onto the MDS map to help interpret the dimensional solutions by visualizing the relationships of words on a map.

In short, both MDS and hierarchical cluster analysis investigated the underlying structure of Japanese beliefs about relational satisfaction with best friends. Specifically, MDS revealed dimensional solutions of Japanese beliefs of satisfaction with best friends. Hierarchical cluster analysis uncovered subgroups of the beliefs about satisfaction with best friends.

\section{Results}

\section{Determining the Dimensional Solution}

In order to determine the number of dimensional solution for Japanese people's beliefs about relational satisfaction with best friends, 
Figure 4-1. Stress values along one to six dimensions of Japanese people's organization of beliefs about satisfaction with best friends.

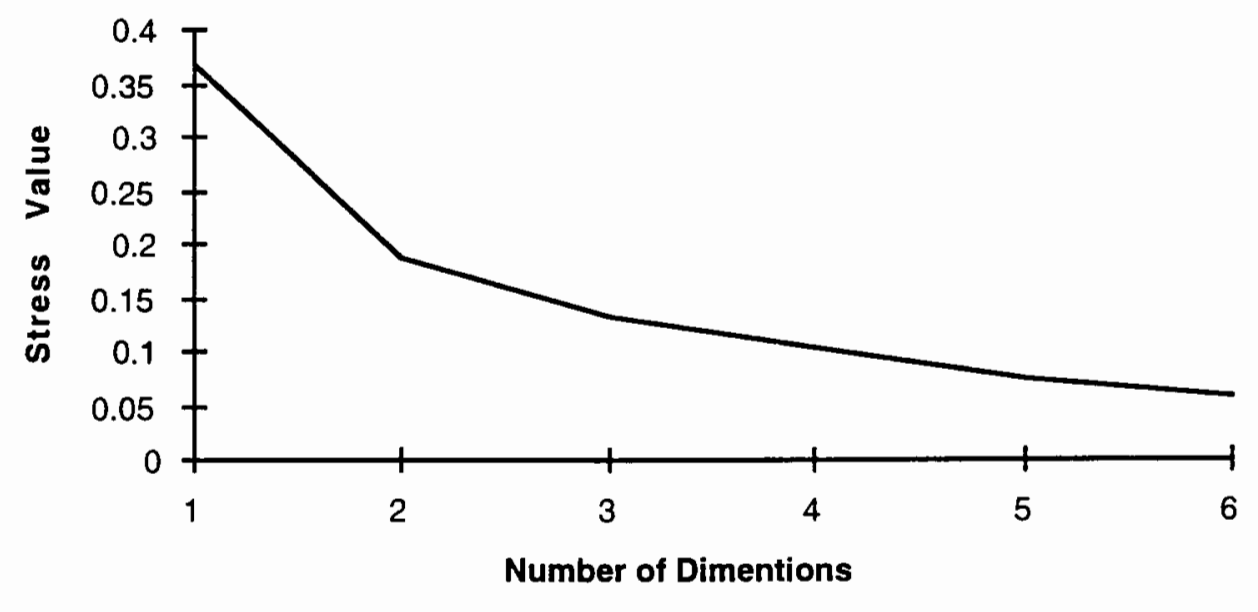

multidimensional solutions for a one to six dimensional solution were examined. While an elbow of the line in the stress plot is one of the keys to determine dimensional solution, the stress plot of the present study did not have a clear curve with an elbow. However, there is not much improvement in stress after four dimensional solution (see Figure 4-1). The stress in a one-dimensional solution was high (.37). Therefore, two through four dimensional maps were produced and dimensional interpretability of each map was explored.

In order to help interpreting two through four dimensional maps, hierarchical cluster analysis was performed (see Figure 4-2). The result of the hierarchical cluster analysis was investigated to determine the best cluster solution for the 39 words. In order to find the best fitting cluster analysis solution, major jumps in the coefficient were investigated. There were four stages which were stages before major jumps: stages $8,20,22$, and 
Figure 4-2. Dendrogram of the clusters of Japanese people's beliefs about relational satisfaction with best friends.

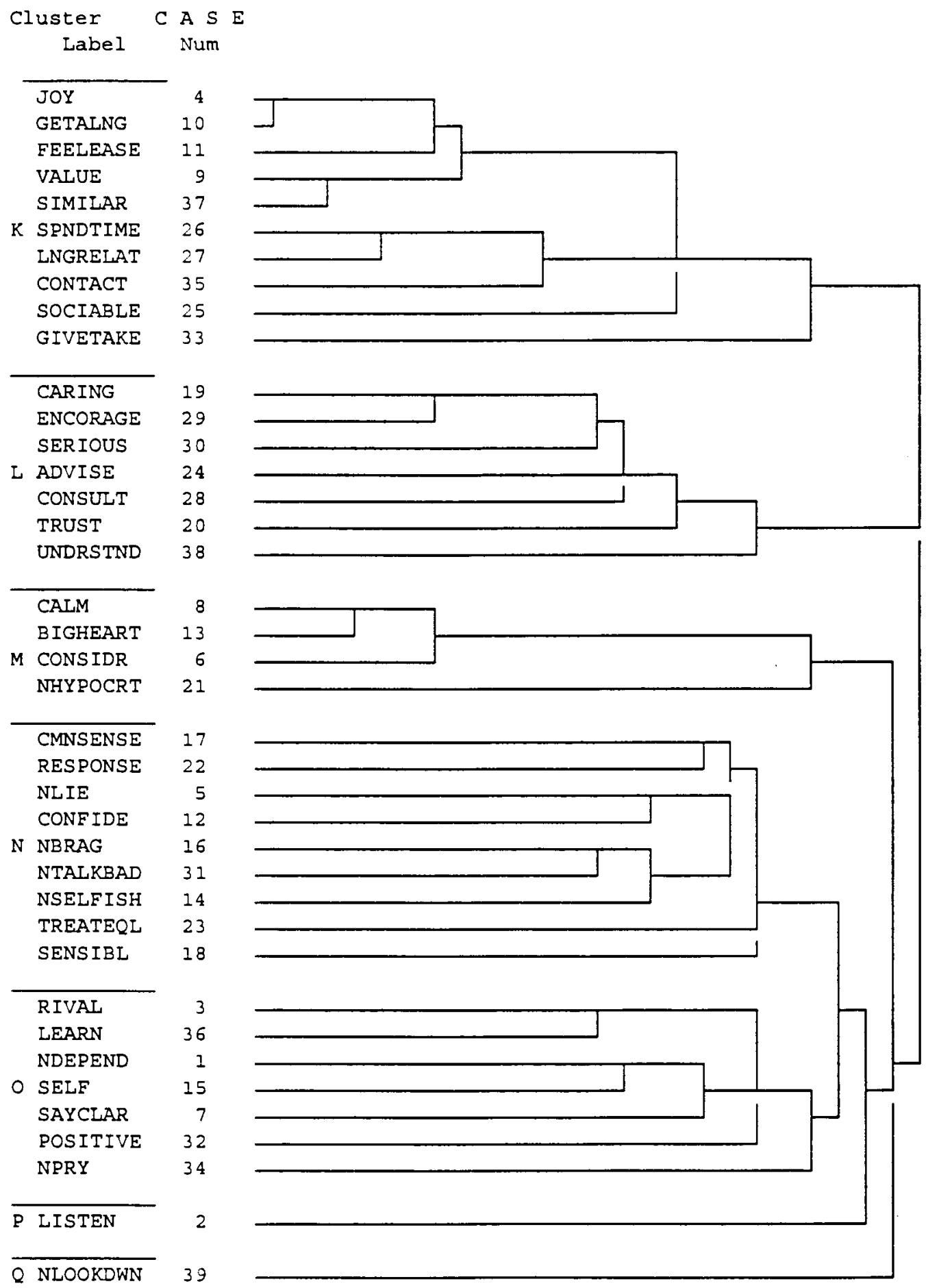


Figure 4-3. Coefficient of agglomeration schedule in order to uncover subgroups of Japanese beliefs about satisfaction with best friends.

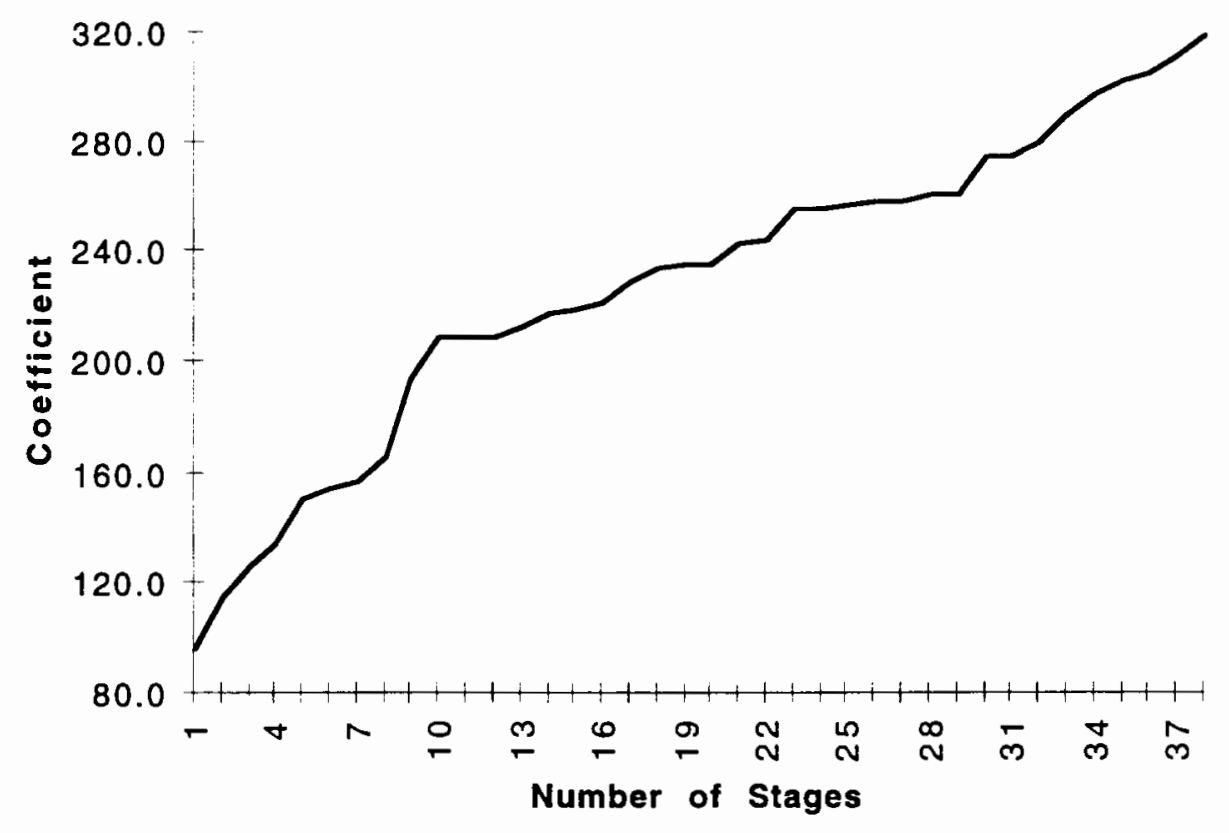

29. The cluster solutions for these stages were the 31-, 19-, 17-, and 10cluster solutions (see Figure 4-3). Since a smaller cluster solution assists dimensional interpretations, a 10-cluster solution seemed appropriate.

Further inspection of the dendrogram suggested that three oneword-clusters could be combined with other clusters in a few steps forward from stage 29. These words were: \#21. Straightforward/Not hypocritical(Sunao de aru), \#33. A give-and-take relationship (Mochitsu motaretsu no kankei de aru), \#34. Does not pry too much (Yokei na koto wo sensaku sinai). Therefore, a 7-cluster solution was chosen for this study. By examining the words in each cluster, Cluster $\mathrm{K}$ was named as companionship, Cluster L as supportiveness, Cluster $\mathrm{M}$ as consideration, 
Cluster $\mathrm{N}$ as integrity, Cluster $\mathrm{O}$ as independence, Cluster $\mathrm{P}$ as openmindedness, and Cluster $Q$ as respect (see Table 4-1). These seven subgroups were superimposed onto two to four dimensional maps in order to help interpretation of the maps.

Table 4-1

Descriptions of the Seven Clusters

\begin{tabular}{|c|c|c|}
\hline Cluster & Name of the cluster & Words \\
\hline Cluster K & companionship & $\begin{array}{l}\text { 4. Joyful to be with, } \\
\text { 9. Has similar values, } \\
\text { 10. A person I get along well with, } \\
\text { 11. Relationship that feels at ease, } \\
\text { 25. Sociable, } \\
\text { 26. A person I spend a long time with, } \\
\text { 27. A person I can have a long-term relationship } \\
\text { with, } \\
\text { 33. A give-and-take relationship, } \\
\text { 35. A relationship in which we keep in contact with } \\
\text { each other, } \\
\text { 37. Has similarities with me }\end{array}$ \\
\hline Cluster L & supportiveness & $\begin{array}{l}\text { 19. Caring, 20. Trustworthy, } \\
\text { 24. Advises me, } \\
\text { 28. A person I can consult about anything, } \\
\text { 29. Encourages me, } \\
\text { 30. Deals with a problem seriously when needed, } \\
\text { 38. Understands me }\end{array}$ \\
\hline Cluster M & consideration & $\begin{array}{l}\text { 6. Considerate, } 8 \text {. Calm, } \\
\text { 13. Big-hearted, } \\
\text { 21. Straightforward/Not hypocritical }\end{array}$ \\
\hline Cluster $\mathrm{N}$ & integrity & $\begin{array}{l}\text { 5. Does not lie, 12. Can confide in, } \\
\text { 14. Not selfish, } \\
\text { 16. Does not brag about him/herself, } \\
\text { 17. Has common sense, } 18 \text {. Sensible, } \\
\text { 22. Has a sense of responsibility, } \\
\text { 23. Treats everyone equal, } \\
\text { 31. Does not speak ill of others }\end{array}$ \\
\hline Cluster $\mathrm{O}$ & independence & $\begin{array}{l}\text { 1. Does not depend on a friend too much, } \\
\text { 3. A rival in a good sense, } \\
\text { 7. Says what he/she thinks clearly, } \\
\text { 15. Has a sense of self , } 32 \text {. Positive, } \\
\text { 34. Does not pry too much, } \\
\text { 36. Has something I can learn }\end{array}$ \\
\hline Cluster P & open-mindedness & 2. Listens to a partner's opinion \\
\hline Cluster Q & respect & 39. Does not look down on me \\
\hline
\end{tabular}


A visual examination revealed that the four dimensional solution was not clearly interpretable while both the two and three dimensional maps were interpretable. Since the three underlying dimensions were meaningful and the stress value of the three dimensional solution was lower (.131) than that of the two dimensional solution (.187), the three dimensional solution was chosen as the solution for Japanese beliefs about relational satisfaction with best friends (see Figure 4-4 and 4-5).

\section{Interpreting the Dimensions}

In order to determine an interpretation of each dimension, three graduate students who were familiar with this study inspected the three dimensional map. Their insights were important in obtaining an accurate interpretation. At the same time, the interpretation needs to be an interpretation lay people agree with. Therefore, eight Japanese undergraduate students who matched the sample population of this study examined the three dimensional map as well. Considering the results of both groups' interpretation, I interpreted the dimensions as follows:

Dimension 1 as Light Hearted ${ }^{1}$-Solidarity vs Serious-Rational (Kigaru, Rentaiteki Keikou vs Majime, Riseiteki Keikou), Dimension 2 as Acceptant-Reliable vs Independent-Positive (Juyou, Kakujitsuteki Keikou vs Jiritsu, Sekkyokuteki Keikou), Dimension 3 as Frank-Supportive vs Modest-Self Controlled (Soccyoku, Shienteki Keikou vs Kenkyo, Jiseiteki Keikou).

1 The word "light hearted" was chosen as a translation of "kigaru," which actually has a meaning of easy to get along with, carefree, and not too serious. 
Figure 4-4. Dimension 1 and 2 in the three-dimensional solution of Japanese people's beliefs about relational satisfaction with best friends.

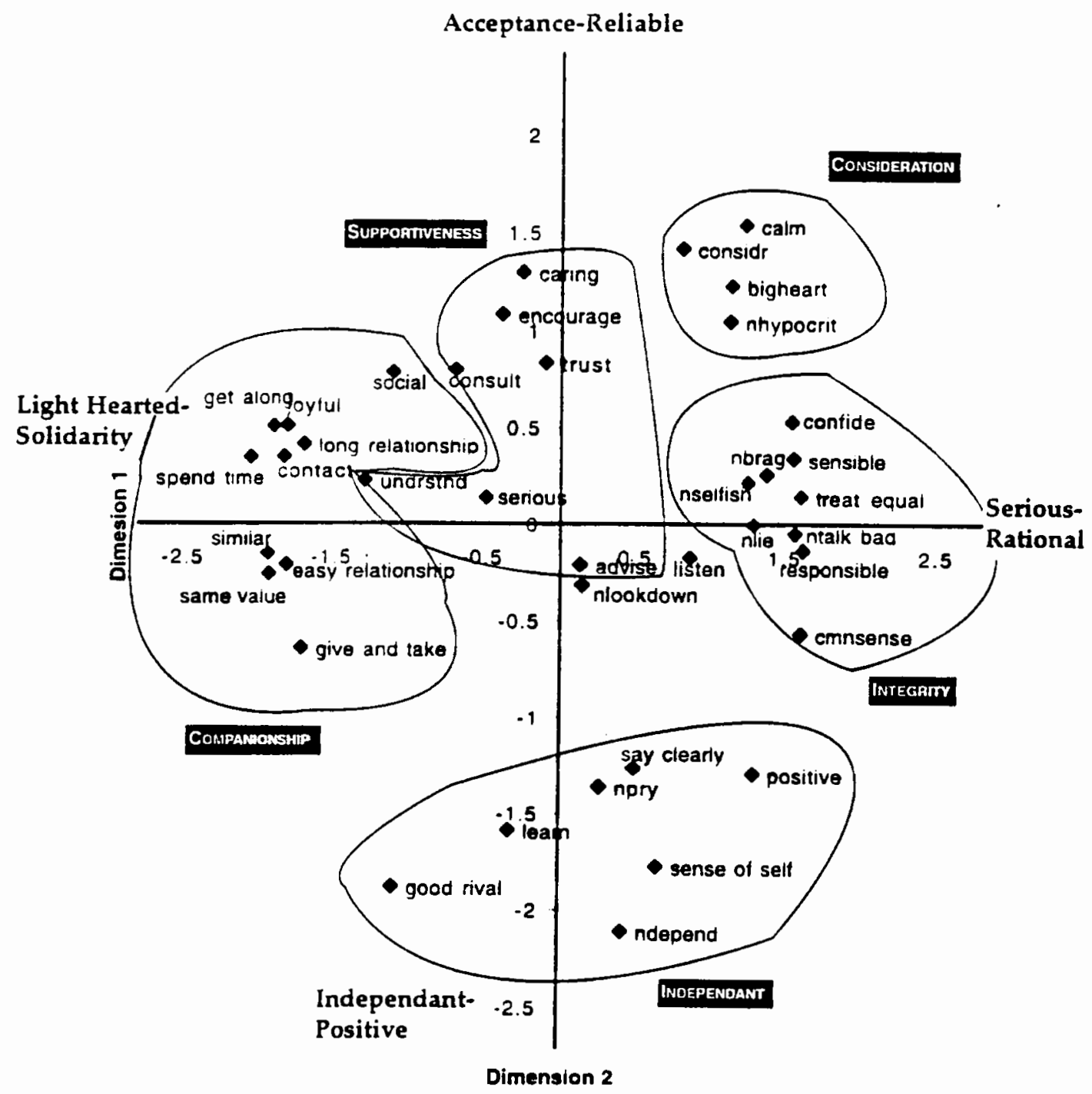


Figure 4-5. Dimension 1 and 3 in the three-dimensional solution of Japanese people's beliefs about relational satisfaction with best friends.

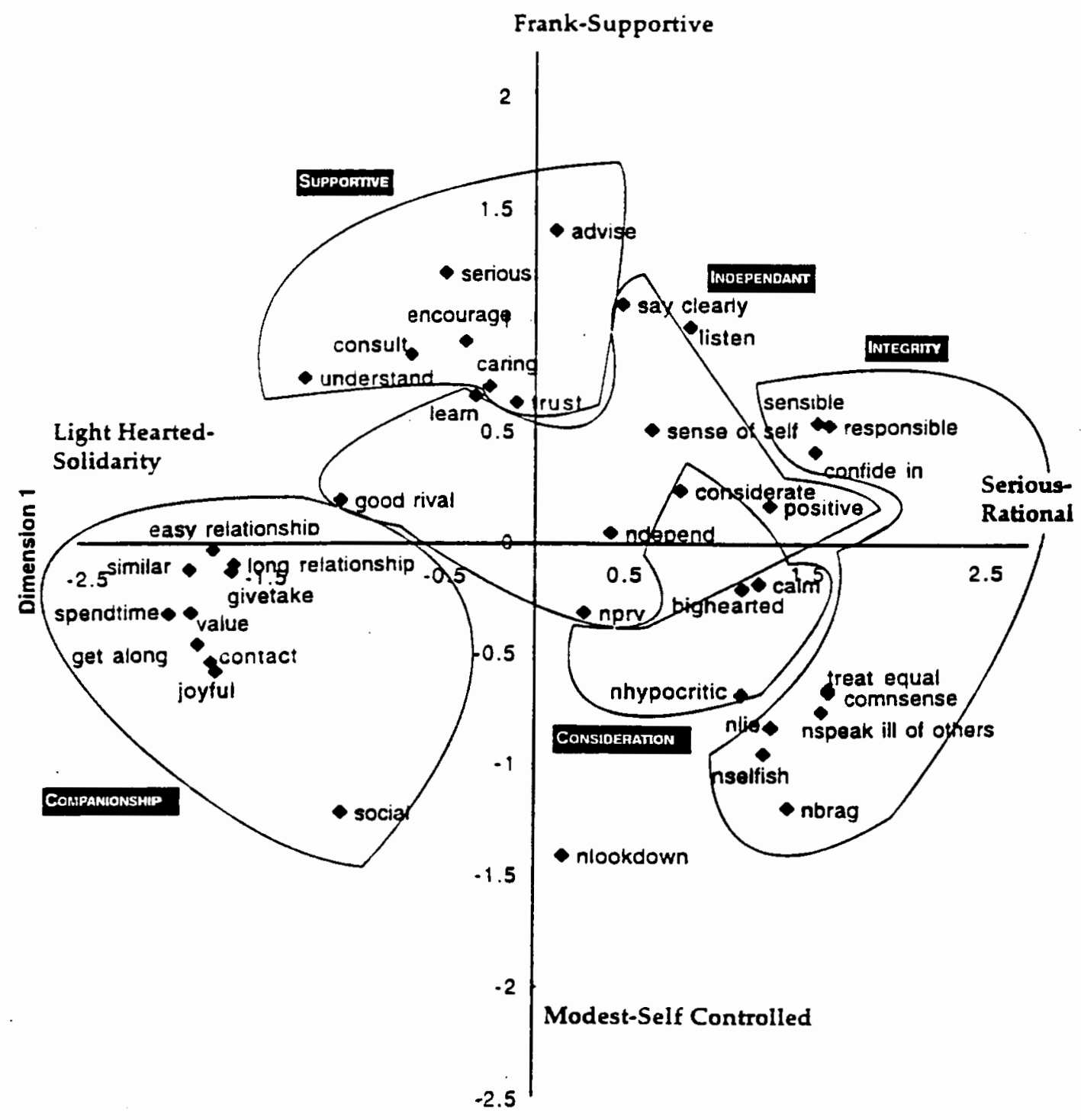

Dimension 3 
Discussion

The three underlying dimensions for Japanese people's beliefs about relational satisfaction with best friends were revealed in this stage. In order to highlight the meaning behind each dimension, and for the purpose of linear presentation, each dimension was discussed separately. However, it is important to note that the three dimensions discussed here are not separate concepts but interrelated as Japanese people's beliefs concerning best friends.

\section{Dimension One}

The first dimension was interpreted as light hearted-solidarity vs serious-rational. This dimension suggests that people's beliefs are related to best friend's desirable characteristics. One end of this dimension includes concepts such as "a person I spend a long time with," "has similarities with me," and "a person I get along well with," and these words represent a light hearted-solidarity evaluation. Cluster $\mathrm{K}$ (Companionship), which emerged from hierarchical cluster analysis, is on this end. It seems that this end of the dimension relates to a best friend's desirable characteristics in an interpersonal relationship, specifically in a best friendship.

On the other hand, the other end of this dimension includes concepts such as "has a sense of responsibility," "has common sense," and "treats everyone equal," and these words represent a serious-rational evaluation. Cluster $\mathrm{N}$ (Integrity) is on this end. It seems that beliefs in this 
end relate to a best friend's desirable characteristics in a social relationship or in general.

This dimension suggests that Japanese people's beliefs about relational satisfaction with best friends are organized by a best friend's desirable characteristics within the interpersonal-social continuum. In other words, people differentiate a best friend's interpersonally desirable qualities such as similarity and closeness from socially desirable qualities such as being responsible and having common sense.

While qualities on the end of a best friend's interpersonal desirability such as "joyful to be with" and "has similarities with me" are supported by previous friendship researchers (e.g., Davis \& Todd, 1985; La Gaipa, 1977), qualities on the other end, friend's social desirability are not. There are two possible explanations. One is that the qualities of social desirability emerged in this particular friendship research because of the focus of this study. Cole and Bradac (1994) proposed different dimensions from previous researchers, and they suggested that " beliefs about satisfaction are organized differently than are people's general beliefs about friendships" (p. 20). Since this study is a replication of Cole and Bradac, it may be possible that people considered the qualities of social desirability as important when being asked beliefs about satisfaction with best friends.

Another explanation is that these qualities of social desirability emerged because of the Japanese collectivistic cultural tendency. Since people in collectivistic cultures tend to see themselves as a member of a group, it may be important for them to hold qualities of a desirable social 
self or group self. In that light those people might also want their best friends to possess a socially desirable qualities.

\section{Dimension Two}

The second dimension was interpreted as acceptance-reliable vs independent-positive. This dimension suggests that Japanese people's beliefs are related to a best friend's dependability. The beliefs of satisfaction such as "calm," "considerate," and "caring" represent an acceptance-reliable evaluation. Cluster M (Consideration) is on this end. It seems that this end represents interdependent qualities people look for from their best friends.

The other end of the second dimension includes beliefs about satisfaction such as "does not depend on a friend too much," "a rival in a good sense," and "has a sense of self," and they represent an independentpositive evaluation. Cluster $O$ (Independence) is on this end. It seems that this end represents independent qualities people look for from their best friends.

This dimension suggests that Japanese people's beliefs about relational satisfaction with best friends are organized by a best friend's dependability within the interdependent and independent continuum. In other words, people differentiate interdependent qualities such as considerate and caring from independent qualities such as a person who has a sense of self and who does not depend on others. 


\section{Dimension Three}

The third dimension was interpreted as frank-supportive vs modest-self controlled. This dimension suggests that Japanese people's beliefs are relate to a best friend's demeanor. The beliefs of satisfaction such as "advises me," "deals with a problem seriously when needed," and "says what s/he thinks clearly" represent a frank-supportive evaluation. Cluster L (supportiveness) is on this end. This end seems to represent active support or an interpersonal demeanor that people look for from their best friends: people want their best friends to provide a strong emotional support.

On the other hand, the beliefs such as "does not looking down on me," "sociable," and "does not brag about him/herself" illustrate a modestself controlled evaluation. There is no particular cluster on this end. However, this end seems to emphasize support by harmonizing with others, or sacrificing his/her ego. In this sense, this end represents passive support or a social demeanor people that look for from their best friends.

This dimension suggests that Japanese people's beliefs are organized by a best friend's demeanor within the interpersonal-social continuum or an active-passive support. In other words, people differentiate qualities which relate to a best friend's interpersonal demeanor or active support (e.g., "advises me," "deals with a problem seriously when needed") from qualities which relate to a best friend's social demeanor or passive support (e.g., "does not looking down on me," "sociable").

While qualities of active support such as helping behavior and understanding were reported as concepts of friendship (e.g., Davis \& Todd, 
1985; La Gaipa, 1977), those of passive support were not reported by previous friendship researchers. There are two possible explanations why Japanese people's beliefs concerning best friends relate to passive support. These explanations are similar to the explanations suggested for Dimension One. One possibility is that the way this study proposed the question (asking beliefs about satisfaction with best friends instead of beliefs about friendships) prompted the responses related to best friends' social demeanor or passive support.

Another possibility is the influence of Japanese collectivistic cultural tendencies. Because the social self is important in a collectivistic cultures such as Japan (Triandis, et al. , 1991), Japanese people may find value in their friends maintenance of social harmony. In order to maintain social harmony, it is crucial that the friends know social norms and present themselves accordingly. This aspect of a best friends' social demeanor or passive support may therefore be highlighted because of this collectivistic cultural tendency of Japan.

\section{Conclusion}

In this stage, Japanese people's underlying dimensions of relational satisfaction with best friends were explored and identified. These dimensions are related to best friend's desirable characteristics, dependability, and demeanor. More specifically, people's beliefs are organized along the dimensions of a best friend's (1) interpersonal-social 
desirable characteristics, (2) interdependent-independent dependability, and (3) interpersonal-social demeanor or active-passive support.

While each dimension has its own unique meaning, it appears that underlying dimensions of Japanese people's beliefs hold the interpersonalsocial aspect. As mentioned in the discussion section, it is unknown whether this interpersonal-social aspect emerged due to the way the question was proposed in this study or as an expression of Japanese collectivistic cultural tendencies or some combination of the two.

However, regarding Japanese subjects in this study, it seems that both social and private aspects concerning satisfaction of friendships are inseparable. As a member of this social structure, Japanese people may not be able to see themselves and their friends as separate from the structure. While previous friendship research has mainly focused on the interpersonal quality of friendship, the findings of this study suggest that people's satisfaction with best friends may not be fulfilled by only interpersonal consideration: people construct their beliefs about relational satisfaction with best friends within the context of both interpersonal and social perspectives. 


\section{Chapter V}

\section{Stage Three}

This chapter describes stage three of the study. The chapter consists of four sections: purpose, methods, results, and discussion sections. In the purpose section, the purpose of stage three is described. In the method section, the sampling frame, instruments, data collection procedure, sample characteristics, and method of analyses are illustrated. In the result section, the results of assessment for proposed dimensional interpretations are presented. The discussion section follows afterwards.

\section{Purpose}

The purpose of this stage is to assess the validity of dimensional interpretations proposed in stage two. While dimensional interpretations were made cautiously by examining the stress and interpretability of the map that emerged through multidimensional scaling analysis (MDS), its validity is reinforced if these interpretations are confirmed by the sample population of this study. Therefore, dimensional interpretations proposed in stage two were examined by Japanese college students. This process is a replication of Cole and Bradac's (1994) study. 
Methods

\section{Sampling Frame}

The sampling frame was drawn from Japanese college students in Japan. The students who fell into one of the categories below were excluded from the sample.

1. Students who previously have stayed overseas a total of more than six months.

2. Students who answered that they have best friends who are not Japanese residents.

3. Students whose age was over 25 years old.

The reasons for above exclusion are the same as stage one which are explained in page 43 .

\section{Instruments}

In order to assess the validity of the dimensional interpretations, Davison (1983) states that one must have measures of the stimuli on each hypothesized attribute. These measures must be obtained by procedures separate from those used to obtain the proximity data on which MDS is based (pp. 189-190). A Likert-format questionnaire was constructed by adapting a Cole and Bradac's (1994) questionnaire in order to obtain measures of the stimuli on each attribute. The 39 beliefs about relational satisfaction with best friends, which are identified in stage one, are the stimuli and the three dimensional interpretations proposed in stage two, 
are the attributes (see Table 3-1 for the 39 beliefs). The interpretations proposed are:

Dimension 1 - Light Hearted-Solidarity vs Serious-Rational (Kigaru, Rentaiteki Keikou vs Majime, Riseiteki Keikou), Dimension 2 - Acceptant-Reliable vs Independent-Positive (Juyou, Kakujitsuteki Keikou vs Jiritsu, Sekkyokuteki Keikou), Dimension 3 - Frank-Supportive vs Modest-Self Controlled (Soccyoku, Shienteki Keikou vs Kenkyo, Jiseiteki Keikou).

For instance, subjects were asked to rate whether the word "does not depend on a friend too much" has light hearted-solidarity or seriousrational meaning in terms of scale one as "clearly illustrates the idea of being light hearted-solidarity" to five as "clearly illustrates the idea of being serious-rational."

If subjects were asked to rate each of the 39 words on a 5-point scale in terms of the 3-dimensional interpretations proposed in stage two, this task would require each subject to answer 117 questions ( 39 words $\times 3$ dimensions) in the same manner. This is a heavy task to ask of a subject. Moreover, simple repetition of a task may distort the accuracy of the data. Therefore, this task was split into two questionnaires; one questionnaire evaluates 20 words (Questionnaire 3A), while other rates the remaining 19 words (Questionnaire 3B) in terms of the 3-dimensional interpretations. Questionnaire 3A and 3B had exactly the same format except the words to be evaluated were different (see Appendix B). 


\section{Data Collection Procedure}

I contacted two college instructors in Japan, describing this research, and asked for their cooperation for data collection. I received their permission to use 30 minutes of class time for the data collection and all data were collected between November and December, 1995.

The same procedure was followed in each class. An instructor introduced me to the students, and I informed them of the purpose of this study in the same manner as stages one and two. Then, students were provided a questionnaire with an informed consent form. Two different questionnaires (Questionnaire $3 \mathrm{~A}$ and $3 \mathrm{~B}$ ) were assigned randomly to the students.

The first page of the questionnaire was an informed consent form and if the students agreed to participate in this study, they were instructed to go on to the next page. After rating all the words in the scale of one to five, the students were asked personal information as well as information about people they considered their best friends. This questionnaire format was the same as the one which was used in stage one.

\section{Sample Characteristics}

Sixty students were asked to complete questionnaire $3 \mathrm{~A}$ ( 26 males, 34 females). Of those, eight students did not match the sampling frame for this study. Therefore, of the 60 questionnaire 3As completed, 52 were used (22 males, 30 females). Fifty four students completed the questionnaire 3B (27 males, 27 females). Three students did not match the sampling frame. 
Therefore, of the 54 questionnaire 3Bs, 51 were used for the analyses (26 males, 25 females).

\section{Methods of Analysis}

Multiple linear regression analyses were performed to assess the dimensional interpretations. Linear regression is most commonly used method to assess the dimensional solutions (Kruskal \& Wish, 1978). Kruskal and Wish explained linear regression as the use of assessment of dimensional solution as follow:

Suppose we have some variables associated with the items which we suspect may have a systematic relationship to position in the configuration. One way to see if it does is to perform a linear multiple regression using this variable as the dependent variable and the coordinates of the configuration as the independent variables. In the compact jargon of statistics, we can refer to this as regressing the variable over the coordinates of the configuration .... What this means is that we seek some weighted combination of the coordinates of the configuration which agrees with or 'explains' the variables as well as possible. (p. 36)

For this study, if the dimensional interpretation proposed in stage two makes sense to the sample population, subjects' ratings of words according to those proposed interpretation should relate to configuration of the 3-dimensional map. Therefore, whether the data, collected by using 5 -point Likert-type scale, has systematic relationship to the coordinates of 
words in the 3-dimensional map or not was examined by using regression analyses.

In order to conduct multiple linear regression analyses, all subjects' ratings of every word on each of the scales were first averaged. These averaged ratings were used as variables. Then, these averaged ratings of the words on each of the three scales were regressed over the coordinates of the words on the 3-dimensional solution that emerged from the MDS analysis.

Kruskal and Wish (1978) proposed two criteria to accept dimensional interpretation;

1) the multiple correlations for the scale must be high, and

2) the scale must have a high regression weight on the dimension of the associated scale but not the others.

As a minimal requirement, the multiple correlation for the scale is statistically significant at the .01 level or better (Kruskal \& Wish, 1978). This study followed their criteria.

\section{Results}

As shown in Table 5-1. the results of multiple linear regression analyses revealed that the Light Hearted-Solidarity vs Serious-Rational scale has a high and statistically significant multiple correlation, $R=.85$, $\mathrm{p}<.001$, strongly correlated to Dimension 1 (the cosine direction for Dimension 1=.867). The Acceptant-Reliable vs Independent-Positive scale has a high and statistically significant multiple correlation, $\mathrm{R}=.89, \mathrm{p}<.001$, 
Table 5-1

Regression Results for Likert-Scale Ratings over Proposed Dimensional $\underline{\text { Interpretations }}$

\begin{tabular}{l|r|r|r|r|r|c}
\hline & Dim 1 & Dim 2 & Dim 3 & R & F & p \\
\hline $\begin{array}{l}\text { Light Hearted- } \\
\text { Solidarity vs } \\
\text { Serious-Rational }\end{array}$ & $\underline{0.867}$ & -0.307 & 0.392 & 0.852 & 30.90 & $<.001^{* *}$ \\
\hline $\begin{array}{l}\text { Acceptant-Reliable } \\
\text { vs Independent- } \\
\text { Positive }\end{array}$ & 0.440 & $\underline{-0.892}$ & 0.101 & 0.887 & 43.25 & $<.001^{* *}$ \\
$\begin{array}{l}\text { Frank-Supportive vs } \\
\text { Modest- } \\
\text { Self Controlled }\end{array}$ & 0.610 & 0.120 & $\underline{-0.783}$ & 0.669 & 9.45 & $<.001^{* *}$ \\
\hline
\end{tabular}

Note. The direction cosine weights were calculated by using standardized regression weights for each dimension.

strongly correlated to Dimension 2 (the cosine direction for Dimension 2=.892). These results met the criteria of Kruskal and Wish (1978).

The Frank-Supportive vs Modest-Self Controlled scale has a moderately high and statistically significant multiple correlation, $R=.67$, $\mathrm{p}<.001$, strongly correlated to Dimension 3 (the cosine direction for Dimension $3=-.783$ ). Since it is desirable to have multiple correlation above .75 (Kruskal \& Wish, 1978), the interpretation of this dimension must be treated with caution.

Discussion

\section{Assessment of Dimensional Interpretations}

From the results of multiple linear regression analyses using Likerttype scale data and the coordinates of the Japanese map, dimensional 
interpretations for Dimension 1, Light Hearted-Solidarity vs SeriousRational, and Dimension 2, Acceptant-Reliable vs Independent-Positive, were strongly supported. Precisely, the findings revealed that subjects differentiated beliefs of relational satisfaction with best friends in terms of Light Hearted-Solidarity vs Serious-Rational(or best friend's interpersonalsocial desirable characteristics) and Acceptant-Reliable vs IndependentPositive (or a best friend's interdependent-independent dependability) dimensions. Furthermore, subjects' way of differentiation was consistent with the dimensional solution proposed in stage two. From the findings above, it seems reasonable to state that Japanese people possess underlying dimensions of Light Hearted-Solidarity vs Serious-Rational(or a best friend's interpersonal-social desirable characteristics) and AcceptantReliable vs Independent-Positive (or a best friend's interdependentindependent dependability) in their beliefs of relational satisfaction with best friends.

The dimensional interpretation for Dimension 3, Frank-Supportive vs Modest-Self Controlled (or a best friend's interpersonal-social demeanor), was moderately supported. One possible reason that Dimension 3 did not receive strong support may be because the range of words along this dimension is rather small compared to other two Japanese dimensions. Coordinates along Dimension 1 vary between -2.04 and 1.63, and coordinates along Dimension 2 vary between -2.11 and 1.54 , while coordinates of Dimension 3 vary between -1.41 and 1.40. In other words, less extreme differences were found along Dimension 3 compared to Dimension 1 or Dimension 2 from the card sorting result. Therefore, it 
might be a more difficult task for the subjects in this stage to rate the words along the Frank-Supportive vs Modest-Self Controlled scale than to rate along the Light Hearted-Solidarity vs Serious-Rational or AcceptantReliable vs Independent-Positive scale.

Another possible reason is that Dimension 1 may be the dominant dimension for Japanese beliefs about relational satisfaction with best friends, making Dimension 3 inadequate as a sole strong predictor of the Frank-Supportive vs Modest-Self Controlled scale. As shown Table 5-1, although each dimension is the highest predictor of each proposed interpretation, Dimension 1 is a predictor of the Acceptant-Reliable vs Independent-Positive scale with a cosine direction $=.440$, and a predictor of the Frank-Supportive vs Modest-Self Controlled scale with a cosine direction $=.610$. These results suggest that Dimension 1 or the Light Hearted-Solidarity vs Serious-Rational dimension is an important factor in Japanese people's beliefs concerning best friends.

In chapter four, I described Dimension 1 as being related to the desirable interpersonal-social characteristics of a best friend and Dimension 3 as being related to the desirable characteristics of a best friend's interpersonal-social demeanor. Both dimensions have an interpersonalsocial continuum. It appears that the differences of these two dimensions lie in the fact that Dimension 1 focuses on the characteristics of best friends in general, whereas Dimension 3 focuses on the characteristics of best friends in relationship to the others. In this sense, Dimension 1 holds a more abstract and Dimension 3 a more specific idea of friendship or Dimension 1 is a dominant dimension and Dimension 3 is a subordinate 
dimension. As a result, the ratings of the Frank-Supportive vs Modest-Self Controlled scale did not correlate solely to Dimension 3 but correlated to Dimension 1 as well.

From the fact that the interpretation of Japanese Dimension 3 does not receive strong support from the sample population, two questions were reconsidered: (1) Is it more appropriate to choose the 2-dimensional solution than the 3-dimension for Japanese beliefs of relational satisfaction with best friends? (2) Is there a more appropriate interpretation for Dimension 3?

Since Dimension 1 and 2 were strongly supported, at least two underlying dimensions for people's beliefs concerning friendship were confirmed. However, this does not prove the 2-dimensional solution is a better solution than the 3-dimensional solution. Additionally, choosing a 2-dimensional solution has its own weakness about representativeness of the data because of the higher stress value (.187). A dimensional solution with lower stress value represents subjects' data better, and in turn, better represents the subject's organization of beliefs of relational satisfaction with best friends. In this sense, the 3-dimensional solution was better than the 2-dimensional solution.

The existence of a better interpretation for Dimension 3 is indeterminable. However, since interpretations of the three dimensions were decided after total of eleven people's inspection of the map, and the interpretations for Dimension 1 and 2 were supported, I suspect the existence of better interpretation for Dimension 3 in this study. 
Finally, I would conclude that a Japanese third dimension seems to exist in the present study. However, it is not as strong a dimension as the first and second dimensions. I believe that replications of this study or conducting further studies on friendship from a relational satisfaction perspective will provide better insight about the presence and an interpretation of a Japanese third dimension as well as, perhaps, other dimensions.

\section{Conclusion}

In this stage, the three dimensional interpretations proposed in stage two were examined by sampling from the same population as stage two. The results revealed that dimensional interpretations for Dimension 1, Light Hearted-Solidarity vs Serious-Rational, and Dimension 2, AcceptantReliable vs Independent-Positive, were strongly supported by the Likerttype scale data. These findings imply that Japanese people possess an underlying structure of Light Hearted-Solidarity vs Serious-Rational and Acceptant-Reliable vs Independent-Positive in their beliefs of relational satisfaction with best friends.

The interpretation for Dimension 3, Frank-Supportive vs ModestSelf Controlled, was moderately accepted. Two reasons that the interpretation of this dimension was not supported strongly were introduced. First, because the range of words along Dimension 3 is rather small in the MDS map, the subjects might have more difficulty rating the words along the Frank-Supportive vs Modest-Self Controlled scale. 
Second, it seems that Dimension 1 is a dominant dimension while Dimension 3 is a subordinate dimension. Therefore, Dimension 3 did not appear to be a solo predictor of the Frank-Supportive vs Modest-Self Controlled scale. Since the interpretation of Dimension 3 FrankSupportive vs Modest-Self Controlled was supported moderately, more research on beliefs about relational satisfaction in friendships is invited. 
Chapter VI

Stage Four

This chapter describes stage four of this study. Stage four examines similarities and differences of underlying dimensions of people's beliefs about relational satisfaction with best friends between Japan and the U.S. The chapter consists of five sections: purpose, methods, results, discussion, and conclusion sections.

\section{Purpose}

Cole and Bradac (1994) explored beliefs about relational satisfaction with best friends using US college students. The present study replicates their study in stages one through three by using Japanese college students as a sample. While similarities and differences of beliefs between Japan and the U.S. were discussed in stage one, this stage attempts to examine similarities and differences of the underlying structure of beliefs about satisfaction with best friends between Japan and the U.S.

In order to explore similarities and differences of the underlying structure between Japan and the U.S, three underlying dimensions of beliefs identified in stage two of this study are compared with the three underlying dimensions identified in Cole and Bradac's (1994) study. If similarities are revealed, these may be viewed as shared dimensions between Japanese and U.S. cultures. If differences are observed, these 
underlying dimensions may be viewed as culturally specific. Since it is assumed that people use the organization of these beliefs to understand and predict the behaviors and traits of their best friends, differences of the underlying structure, if any, may be an obstacle in developing intercultural friendships between people from Japan and the U.S. Therefore, it is important to compare underlying dimensions of beliefs about relational satisfaction with best friends between Japan and the U.S.

In this stage, the following research question is posed:

RQ4: How similar is the organization of beliefs about satisfaction with best friends between Japan and the United States?

Method

Cole and Bradac (1994) proposed a 3-dimensional solution for U.S. people's beliefs about relational satisfaction with best friends, and those dimensions are interpreted as follows:

Dimension 1 as spontaneous-active vs stable-passive,

Dimension 2 as rational-reserved vs emotional-intimate,

Dimension 3 as ambitious-active vs easy-going-unassuming.

I proposed a 3-dimensional solution for Japanese people's beliefs about relational satisfaction with best friends in stage two of this study, and those dimensions are interpreted as follows:

Dimension 1 as Light Hearted-Solidarity vs Serious-Rational (Kigaru, Rentaiteki Keikou vs Majime, Riseiteki Keikou), 
Dimension 2 as Acceptant-Reliable vs Independent-Positive (Juyou, Kakujitsuteki Keikou vs Jiritsu, Sekkyokuteki Keikou),

Dimension 3 as Frank-Supportive vs Modest-Self Controlled (Soccyoku, Shienteki Keikou vs Kenkyo, Jiseiteki Keikou).

In order to explore similarities and differences of the above underlying dimensions between Japanese and U.S. subjects, similarity between Japanese and U.S. beliefs was first investigated. If similar beliefs were found, then similarities and differences of underlying dimensions were investigated by using the coordinates ${ }^{1}$ of these similar beliefs on the maps that emerged from multidimensional scaling (MDS) analyses.

\section{Determining Pairs between Japanese and U.S. Beliefs}

In order to investigate similarity between Japanese and U.S. beliefs, four Japanese people, who were fluent in both Japanese and English and who were familiar with this study, individually tried to match 39 Japanese beliefs identified in this study with 43 beliefs identified in Cole and Bradac's (1994) study. Pairs of beliefs were chosen under the following criteria:

1) more than three people had to report the same pair as similar, and

2) one belief from one culture has to match with only one belief from another culture.

\footnotetext{
${ }^{1}$ The coordinates used here were coordinates of the original dimensional solution. The coordinates along cosine directions and the coordinates of rotated dimensional solution were also examined and similar results were found.
} 
From the criteria above, fourteen pairs of beliefs were determined as similar to each other and chosen to use for the analyses. Table 6-1 shows these 14 pairs side by side. These 14 beliefs were moderately scattered in each of three dimensions in both Japanese and U.S. maps.

Table 6-1

Similarity between the Japanese and U.S. Beliefs about Relational Satisfaction with Best Friends

\begin{tabular}{l|l}
\hline \multicolumn{1}{c|}{ Japanese Beliefs } & \multicolumn{1}{c}{ U.S. Beliefs } \\
\hline $\begin{array}{l}\text { 1. Does not depend on a friend too much } \\
\text { (Aite ni izon shisuginai ) }\end{array}$ & 27. independent \\
\hline 4. Joyful to be with (Issyoni ite tanoshii) & 22. fun to be around \\
\hline 5. Does not lie (Uso wo tsukanai) & 26. honest \\
\hline 6. Considerate (Omoiyari ga aru) & $\begin{array}{l}\text { 10. caring, kind, and } \\
\text { compassionate }\end{array}$ \\
\hline $\begin{array}{l}\text { 15. Has a sense of self } \\
\text { (Jibun to iumono wo motteiru) }\end{array}$ & 13. confident \\
$\begin{array}{l}\text { 16. Does not brag about him/herself } \\
\text { (Jiman wo shinai) }\end{array}$ & 35. not self-absorbed \\
\hline $\begin{array}{l}\text { 17. Has common sense (Joushiki ga aru) } \\
\text { 21. Straightforward/Not hypocritical } \\
\text { (Sunao de aru) }\end{array}$ & 40. practical \\
\hline $\begin{array}{l}\text { 25. Sociable (Tsukiai ga ii) } \\
\text { 27. A person I can have a long-term relationship } \\
\text { with (Nagai tsukiai ga dekiru) }\end{array}$ & 4. admits mistakes \\
\hline $\begin{array}{l}\text { 32. Positive (Maemuki de aru) } \\
\text { 34. Does not pry too much } \\
\text { (Yokei na koto wo sensaku sinai) }\end{array}$ & 6. ambitious \\
\hline $\begin{array}{l}\text { 36. Has something I can learn } \\
\text { (Watashi ga manaberu nanika wo motteiru) }\end{array}$ & 11. complects me \\
\hline $\begin{array}{l}\text { 38. Understands me } \\
\text { (Watashi no koto wo rikai shiteiru) }\end{array}$ & 30. intimate \\
\hline
\end{tabular}

Note. See Table 3-1 for detailed descriptions of Japanese beliefs and Appendix E for those of the U.S. 


\section{Conducting Regression Analyses}

Fourteen pairs of beliefs were reported as similar. Using these beliefs, multiple linear regression analyses were conducted in order to investigate relationships between Japanese and U.S. underlying dimensions of beliefs about relational satisfaction with best friends. Regression analysis examines whether a certain variable predicts other variables (Shinmura, 1995). In this stage, the question of whether any of the Japanese dimensions can predict any of the U.S. dimensions and vice versa was examined.

The coordinates of 14 Japanese beliefs emerged from the MDS analysis and they were used as Japanese data. There were three coordinates for each belief since a three dimensional structure was chosen as a dimensional solution in stage two. For U.S. data, the coordinates of the 14 U.S. beliefs were estimated from the maps presented in Cole and Bradac's (1994) study. There were three coordinates for each U.S. belief since Cole and Bradac (1994) chose three dimensionality as a solution. By using these coordinates, each U.S. dimension was regressed over the three Japanese dimensions. In the same manner, each of the Japanese dimensions was regressed over the three U.S dimensions. As a result, a total of six regression analyses were performed.

Results

As shown in Table 6-2, the results of multiple linear regression analyses revealed that the Japanese Dimension 1 has a high and statistically 
significant multiple correlation, $\mathrm{R}=.80, \mathrm{p}=.013$, with a strong correlation to the U.S. Dimension 2 (the cosine direction for the U.S. Dimension 2=-.995). The Japanese Dimension 2 has a high and statistically moderately significant multiple correlation, $\mathrm{R}=.75, \mathrm{p}=.035$, with a strong correlation to the U.S. Dimension 3, (the cosine direction for the U.S. Dimension 3=.888). The Japanese Dimension 3 does not have a significant multiple correlation.

As shown in Table 6-3, the results of multiple linear regression analyses revealed that the U.S. Dimension 1 does not have a significant multiple correlation. The U.S. Dimension 2 has a high and statistically significant multiple correlation, $\mathrm{R}=.80, \mathrm{p}=.013$, with a strong correlation to the Japanese Dimension 1, (the cosine direction for the Japanese Dimension 1=-.989). The U.S. Dimension 3 has a high and statistically moderately significant multiple correlation, $\mathrm{R}=.74, \mathrm{p}=.040$, with a strong correlation to the Japanese Dimension 2, (the cosine direction for the Japanese Dimension 2=-.859).

Table 6-2

Regression Results for U.S. Dimensions over Japanese Dimensions

\begin{tabular}{|c|c|c|c|c|c|c|}
\hline & $\begin{array}{l}\text { US Dim } \\
1\end{array}$ & $\begin{array}{l}\text { US Dim } \\
2 \\
\end{array}$ & US Dim 3 & $\overline{\mathrm{R}}$ & $\bar{F}$ & $p$ \\
\hline Japanese Dim 1 & -0.083 & -0.995 & -0.061 & 0.80 & 6.00 & $0.013^{*}$ \\
\hline Japanese Dim 2 & 0.450 & 0.097 & -0.888 & 0.75 & 4.26 & $0.035^{*}$ \\
\hline Japanese Dim 3 & 0.035 & 0.377 & $\overline{0.927}$ & 0.61 & 1.96 & 0.185 \\
\hline
\end{tabular}

Note. The direction cosine weights were calculated by using standardized regression weights for each dimension. 
Table 6-3

Regression Results for Japanese Dimensions over U.S. Dimensions

\begin{tabular}{l|r|r|r|r|r|l}
\hline & $\begin{array}{c}\text { Japanese } \\
\text { Dim 1 }\end{array}$ & $\begin{array}{c}\text { Japanese } \\
\text { Dim 2 }\end{array}$ & $\begin{array}{c}\text { Japanese } \\
\text { Dim 3 }\end{array}$ & R & F & $p$ \\
\hline US Dim 1 & -0.312 & -0.924 & -0.221 & 0.53 & 1.29 & 0.329 \\
\hline US Dim 2 & -0.989 & 0.111 & 0.096 & 0.80 & 6.04 & $0.013^{*}$ \\
\hline US Dim 3 & 0.000 & -0.859 & $\underline{0.5128}$ & 0.74 & 4.05 & $0.040^{*}$ \\
\hline
\end{tabular}

Note. The direction cosine weights were calculated by using standardized regression weights for each dimension.

From the above results, a strong relationship between the Japanese Dimension 1 and the U.S. Dimension 2 was revealed. A moderately strong relationship between the Japanese Dimension 2 and the U.S. Dimension 3 was also observed. Japanese Dimension 3 did not reveal a strong relationship with any U.S. dimensions. U.S. Dimension 1 did not reveal a strong relationship with any Japanese dimensions.

\section{Discussion}

The results of multiple linear regression analyses, using coordinates of 14 pairs from Japanese and U.S. beliefs about satisfaction with best friends, revealed that Japanese Dimension 1 strongly related to U.S. Dimension 2, and Japanese Dimension 2 related to U.S. Dimension 3. However, there is no Japanese equivalent dimension to U.S. Dimension 1. Also, there is no equivalent U.S. dimension to Japanese Dimension 3. Table 6-4 shows the comparison of these dimensions. 
The results suggests that Japanese Dimension 1, light heartedsolidarity vs serious-rational, related to U.S. Dimension 2, rationalreserved vs emotional-intimate. In stage two, I interpreted Japanese Dimension 1 as a best friend's desirable characteristics, with one end (named as light hearted-solidarity) related to interpersonally desirable characteristics, while another end (named as serious-rational) related to socially desirable characteristics. It seems that U.S. Dimension 2 presents a similar idea, with one end (named as emotional-intimate) related to interpersonally desirable characteristics, while the other end (named as rational-reserved) relates to socially desirable characteristics.

It seems that Japanese and U.S. people have a similar underlying idea of a best friend's desirable characteristics. In other words, people from both cultures differentiate interpersonal aspects of a friend's desirable characteristics from social aspects.

Table 6-4

Comparison of Interpretations between Japanese and U.S. Underlying Dimensions

\begin{tabular}{l|l}
\hline Japanese Dimensions & U.S. Dimension \\
\hline \hline No equivalent dimensions found & $\begin{array}{l}\text { 1. spontaneous-active vs stable- } \\
\text { passive }\end{array}$ \\
\hline $\begin{array}{l}\text { 1. light hearted-solidarity vs } \\
\text { serious-rational }\end{array}$ & $\begin{array}{l}\text { 2. rational-reserved vs emotional- } \\
\text { intimate }\end{array}$ \\
\hline $\begin{array}{l}\text { 2. acceptant-reliable vs } \\
\text { independent-positive }\end{array}$ & $\begin{array}{l}\text { 3. ambitious-assertive vs easy- } \\
\text { going-unassuming }\end{array}$ \\
$\begin{array}{l}\text { 3. frank-supportive vs modest-self } \\
\text { controlled }\end{array}$ & No equivalent dimensions found \\
\hline
\end{tabular}


It is important to note that previous researchers (e.g., Davis \& Todd, 1985; La Gaipa, 1977) reported interpersonal aspects of a best friend's desirable characteristics such as sharing similarities and being intimate as important qualities of friendships. However, social aspects of a friend's characteristics, such as having common sense or being serious, were not reported as key factors for friendship. One possible reason that both Japanese and U.S. beliefs include social aspects is that both Cole and Bradac's (1994) and this study explored beliefs about satisfaction of friendships rather than beliefs about friendships. Subjects' beliefs about satisfaction of friendships may be different from beliefs about friendships, including both social and interpersonal aspects of best friend's desirable characteristics.

The results also reported that Japanese Dimension 2, acceptantreliable vs independent-positive, related to U.S. Dimension 3, ambitiousassertive vs easy-going-unassuming. In stage two, I interpreted Japanese Dimension 2 as a best friend's dependability, with one end (named as acceptant-reliable) related to interdependent while the other end (named as independent-positive) related to an independent quality of dependability. It seems that U.S. Dimension 3 presents a similar idea, with one end (named as easy-going-unassuming) relates to interdependence, while another end (named as ambitious-assertive) relates to independence.

It seems that Japanese and U.S. people have a similar underlying idea of a best friend's dependability. In other words, people from both cultures differentiate a friend's interdependent qualities from independent qualities. 
Japanese Dimension 3, frank-supportive vs modest-self controlled, was not matched by any U.S. dimensions. I interpreted this dimension as a best friend's interpersonal-social demeanor or active-passive support. The finding that there is no equivalent underlying dimension of best friend's demeanor or support in the U.S. dimension is somewhat consistent with a cultural tendency of Japan as collectivistic and the U.S. as an individualistic culture.

In collectivistic cultures, people tend to emphasize the importance of the group more than in individualistic cultures (Hofstede, 1984). In such cultures, people value social identity or group self. From this collectivistic tendency, it makes sense that Japanese beliefs about satisfaction with best friends can be distinguished between a best friend's demeanor or support. Moreover, one end of this continuum represents social demeanor or passive support (named as modest-self controlled). This end seems to reflect the idea that Japanese people want their best friends to harmonize with society.

As an individualistic culture, U.S. culture values uniqueness and individuality more than collectivistic cultures. From this viewpoint, U.S. people may not have an organization of a best friend's level of demeanor or support in their beliefs about satisfaction with best friends. As a result, there is no similar dimension of Japanese frank-supportive vs modest-self controlled dimension in the U.S. dimensions. However, since this dimension was not strongly supported by the Likert-type scale data in stage three, further investigation is needed to make strong conclusions regarding this issue. 
The U.S. first dimension, spontaneous-active vs stable-passive, was not related to any Japanese dimensions. Cole and Bradac (1994) interpreted this dimension as a level of activity. One end of this U.S. first dimension, spontaneous-active, includes qualities such as "active/energetic,"

"activities director" which I interpreted as qualities related to stimulus in stage one. In stage one, I argued that a stimulating friendship seemed important for U.S. people but not necessarily for Japanese people. Then, it makes sense that a dimension that has one end called spontaneous-active does not exist as a Japanese people's underlying dimension of beliefs about satisfaction with best friends.

In addition, the U.S. dimension of a level of activity may also be consistent to the U.S. cultural tendency of a "doing" culture identified by Kluckhohn and Strodtbeck (1961). According to Kluckhohn and Strodtbeck (1961), cultures vary in whether they value being, doing, or becoming. Cultures which value "doing" emphasize "activities outside the individual" (Infante, Andrew, \& Womack, 1993, p. 433). U.S culture is considered as a "doing" culture and the idea of "doing" has impact on every aspect of U.S. lifestyle (Stewart, 1972). This cultural value may influence U.S. people's organization of beliefs concerning friendships. As a result, U.S. people may consider the level of activity as an underlying dimension in beliefs about satisfaction with friends.

Similarities of two of the underlying dimensions between Japan and the U.S. presented here suggest that both Japanese and U.S. people may use similar organization to understand and predict the behaviors and traits of their best friends. The differences between the two cultures suggest that 
Japanese and U.S. people may use different organization to understand and predict their friendships.

Although two of three underlying dimensions from the two cultures were similar, it does not prove that it is easy to develop friendship between Japanese and U.S. people. Since the underlying dimensions are interrelated and cannot exist separately, differences of interpretation may distress intercultural friendships. For instance, the Japanese belief "joyful to be with" belongs to a light hearted-solidarity, acceptance-reliable, and modest-self controlled quadrant, whereas its paired word from the U.S. "fun to be around" belongs to a spontaneous-active, emotional-intimate, and easy going-unassuming quadrant. Even though this pair of words seems similar, "joyful" to Japanese people has a quality of modest-self controlled over frank-supportive while "fun" to U.S. people has a quality of spontaneous-active over stable-passive. As a result, when Japanese and U.S. friends agree to do something fun together, the Japanese style of fun can be too boring for U.S. people because it is self-controlled while the U.S. style of fun can be too crazy for Japanese because of its spontaneity.

\section{Conclusion}

In this stage, underlying dimensions of beliefs about relational satisfaction with best friends between Japan and the U.S. were compared. The results revealed that there are similarities in underlying dimensions of beliefs about satisfaction with best friends between Japan and the U.S. The similarities related to the best friend's interpersonal-social desirable 
characteristics and interdependent-independent dependability. The results also showed that only U.S. people have an underlying dimension related to "a best friend's perceived level of activity." These findings were explained by cultural tendency of uncertainty-avoidance (Japan as high and the U.S. as low in uncertainty-avoidance) and the "doing" culture of the U.S. The results also showed that only Japanese have an underlying dimension of a best friend's interpersonal-social demeanor or activepassive supportiveness. The findings were explained by cultural tendencies of individualism-collectivism (Japan as collectivism and the U.S. as individualism).

There are several limitations in this stage. First, 14 pairs of beliefs were used for the analyses of this stage. The three underlying dimensions emerged from the relationships of all 39 Japanese beliefs for Japanese data. In the same manner, the U.S. three dimensions emerged from the relationships of 43 beliefs about satisfaction with friends. However, only 14 words were used for the analyses. Therefore, although the 14 selected pairs were scattered across the three dimensions in each data, using only 14 similar words may have some influence on comparing the dimensions. Second, each pairs hold similar beliefs about satisfaction with best friends. However, since they are not exactly the same beliefs, examining dimensions by comparing the 14 pairs of beliefs may have some impact on the results. In short, it is important to treat the results presented here with caution.

Third, while some researchers (Kruskal \& Wish, 1978; Davison, 1983) introduced the use of regression analyses in order to assess the 
validity of interpretation of underlying dimensions in the MDS map, I have not found a study that used this technique to compare dimensions of different maps. If valid, this method gives an opportunity to empirically compare dimensions of different maps. However, the validity of the use of regression analysis in order to compare underlying dimensions of different MDS maps is unknown at this point. Therefore, the results using this method weakened the validity of the findings and discussions in this stage.

Despite the limitations above, the present stage gives some insight on possible obstacles in intercultural friendships between Japan and the U.S. Knowing similarities and differences of organizations of beliefs about satisfaction with best friends between the two cultures may help understanding and predicting the behaviors and traits of Japanese friends for U.S. people and vice versa. 
Chapter VII

\section{Stage Five}

This chapter describes stage five of the present study. Stage five examines gender influence on Japanese beliefs about relational satisfaction with best friends. The chapter consists of five sections: purpose, methods, results, discussion, and conclusion sections.

Although Cole and Bradac (1994) examined gender differences on best friendship, this study did not replicate their method. Therefore, my results are not comparable with their results.

\section{Purpose}

Purpose of this stage is to examine gender influence on Japanese people's beliefs about relational satisfaction with best friends. While previous research has investigated gender differences associated with friendships (e.g., Albert \& Moss, 1990; Pleck, 1975), the results are not always consistent. Moreover, most of these studies have been conducted in the U.S., mainly drawing samples from the dominant white population (e.g., Elkins \& Peterson, 1993; Rose, 1985).

Only a few studies investigated gender issues related friendships in Japan (e.g., Enomoto, 1987; Moroi, 1989). In order to gain better understanding about gender influence on friendships, this stage explores whether Japanese men and women have different emphases in their 
concepts of relationships with best friends, depending on their own gender and/or their best friend's gender. Two research questions are posed:

RQ5: How does people's own gender influence their beliefs about satisfaction with best friends in Japan?

RQ6: How does people's best friend's gender influence their beliefs about satisfaction with these friends in Japan?

Method

\section{Sampling Frame}

The sampling frame was drawn from Japanese college students in Japan. The students who fell into one of the categories below were excluded from the sample.

1. Students who previously have stayed overseas a total of more than six months.

2. Students who answered that they have best friends who are not Japanese residents.

3. Students whose age was over 25 years old.

The reasons for above exclusion are the same as stage one which are explained in page 43 .

\section{Instruments}

The instruments for this stage were included in a questionnaire used in stage two (see Appendix B). After agreeing to participate in this study and engaging in card sorting task, students were instructed to review 
a list of 39 words. The words in the list are the 39 words selected as Japanese beliefs of relational satisfaction with best friends in stage one.

Students were, then, asked to select the five words that they think were most important to satisfy their relationships with a best friend of the same sex. Next, they chose the five words that they think were the most important words to describe satisfying relationships with a best friend of the opposite sex.

Students were instructed that they could choose the same words in both relational contexts if they thought this was appropriate. They were also told that they could choose the item "I cannot choose any of the words above when I think of relational satisfaction with the same (or opposite) sex best friends" and were asked to report the reason if possible.

\section{Data Collection Procedure}

The questions for this stage were included in a questionnaire used for stage two. Hence, the data collection procedure was exactly the same as stage two reported on page 69.

\section{Sample Characteristics}

A total of 352 Japanese students from three colleges participated in this stage (193 females, 159 males). Since this stage is a subset of stage two, the total number of participants for this stage is identical to stage two. With these students, 55 did not match the sampling frame of this study or had missing information needed. In addition, sixteen students (10 females, 6 males) who did not choose the words concerning opposite-sex best 
friends, were also excluded. Therefore, the sample of stage five consisted of 281 Japanese undergraduate students (151 females, 130 males).

\section{Clustering Words}

Subjects chose five words out of 39 words which they thought important when they think of relational satisfaction for each context (same sex, opposite sex). Since comparing 39 words as variables is not feasible and may lead to the semantic argument rather than their concepts, the 39 words were clustered into groups. In addition, since it is assumed that some of these words share similar concepts, it is important to group them instead of treat them as separate concepts.

In order to cluster the 39 words, the result of hierarchical cluster analysis was used. Hierarchical cluster analysis was performed based on card-sorting results, and 7-cluster solution was proposed in stage two (see page 74 and Table 7-1). For analysis of this stage, the same 7-cluster solution was adapted in order to investigate gender influence on Japanese people's beliefs of relational satisfaction with best friends.

While subjects reported five words for each context (same sex, opposite sex), the data was converted into the number of words chosen from each of the seven clusters. For instance, if a subject reported \#4 (joyful to be with), 9 (has similar values), 10 (a person I get along well with), 20 (trustworthy), and 14 (not selfish) as five important words for the same sex best friends, this data was converted as three words from Cluster $\mathrm{K}$ (companionship), one word from Cluster L (supportiveness), one word from Cluster $\mathrm{N}$ (integrity), and zero word from the other clusters. 
Table 7-1

Descriptions of the Seven Clusters

\begin{tabular}{|c|c|c|}
\hline Cluster & Name of the cluster & Words \\
\hline Cluster K & companionship & $\begin{array}{l}\text { 4. Joyful to be with, } \\
\text { 9. Has similar values, } \\
\text { 10. A person I get along well with, } \\
\text { 11. Relationship that feels at ease, } \\
\text { 25. Sociable, } \\
\text { 26. A person I spend a long time with, } \\
\text { 27. A person I can have a long-term relationship } \\
\text { with, } \\
\text { 33. A give-and-take relationship, } \\
\text { 35. A relationship in which we keep in contact with } \\
\text { each other, } \\
\text { 37. Has similarities with me }\end{array}$ \\
\hline Cluster L & supportiveness & $\begin{array}{l}\text { 19. Caring , 20. Trustworthy, } \\
\text { 24. Advises me, } \\
\text { 28. A person I can consult about anything, } \\
\text { 29. Encourages me, } \\
\text { 30. Deals with a problem seriously when needed, } \\
\text { 38. Understands me }\end{array}$ \\
\hline Cluster M & consideration & $\begin{array}{l}\text { 6. Considerate, } 8 \text {. Calm, } \\
\text { 13. Big-hearted, } \\
\text { 21. Straightforward/Not hypocritical }\end{array}$ \\
\hline Cluster $\mathrm{N}$ & integrity & $\begin{array}{l}\text { 5. Does not lie, 12. Can confide in, } \\
\text { 14. Not selfish, } \\
\text { 16. Does not brag about him/herself, } \\
\text { 17. Has common sense, } 18 \text {. Sensible, } \\
\text { 22. Has a sense of responsibility, } \\
\text { 23. Treats everyone equal, } \\
\text { 31. Does not speak ill of others }\end{array}$ \\
\hline Cluster $\mathrm{O}$ & independence & $\begin{array}{l}\text { 1. Does not depend on a friend too much, } \\
\text { 3. A rival in a good sense, } \\
\text { 7. Says what he/she thinks clearly, } \\
\text { 15. Has a sense of self, } 32 \text {. Positive, } \\
\text { 34. Does not pry too much, } \\
\text { 36. Has something I can learn }\end{array}$ \\
\hline Cluster $\mathrm{P}$ & open-mindedness & 2. Listens to a partner's opinion \\
\hline Cluster Q & respect & 39. Does not look down on me \\
\hline
\end{tabular}

\section{Methods of analysis for the gender influence}

In order to examine whether there is an overall differences in emphasis on beliefs depending on gender differences of subjects and/or 
their best friends (subject's gender, their friend's gender, and the interaction of their gender and their friend's gender), doubly repeated measures multivariate analysis of variance (MANOVAs) were performed. According to Hair, Anderson, Tatham and Grablowsky (1979), multivariate analysis of variance (MANOVA) examines "the relationship between a combination of two or more dependent response measures, coordinate presumed to be metrically-scaled, and a set of predictor variables which are nonmetric (categorical)" (p. 144). When "each subject has multiple variables measured at multiple times," this is called a doubly multivariate repeated measures design (Norusis, 1994, p. 132). Since my subjects reported five important words in two different conditions (when thinking of same sex best friends and opposite sex best friends), a doubly repeated measures multivariate design was chosen.

In short, gender influence was tested with Doubly Repeated Measures MANOVAs under the following conditions. Dependent measures were number of words chosen from each of the seven clusters resulted from the hierarchical cluster analysis. A between-subject factor was subject's gender (male or female), and a within-subject factor was subject's friend's gender in relation to the subject (same sex or opposite sex).

Since overall significant differences were observed by Doubly Repeated Measures MANOVAs, a repeated measures analysis of variance (ANOVA) was performed for each of the seven clusters. This procedure specified clusters which influenced overall gender differences. For each cluster, condition was the same: subject sex as a between factor and 
relational context as a within factor. For example, in order to test gender effect on Cluster K (companionship), whether the mean score of Cluster K was the same for both male and female subjects, for both same sex and opposite sex friends, and for male and female subjects with relationship to the sex of best friends was tested.

When a significant interaction effect was found for a subject's gender by his/her friend's gender in Repeated Measures ANOVA for a cluster, Paired-Samples T Test and Independent-Samples T Test was performed. These tests were conducted in order to specify whether male or female subjects showed a significant difference depending on their relational context (same or opposite sex best friends).

\section{Results}

\section{Overall Effect}

The Doubly Repeated Measures MANOVAs revealed a significant main effect for subject's gender; $F(7,273)=3.82, \mathrm{p}=.001$, Wilks's $\lambda=.91$, and a significant main effect for friend's gender; $F(7,273)=4.33, \mathrm{p}<.001$, Wilks's $\lambda=.90$. The Doubly Repeated Measures MANOVA also revealed a significant interaction effect on subject's gender by friend's gender; F (7, $273)=5.77, \mathrm{p}<.001$, Wilks's $\lambda=.87$. Since all of the overall effects were revealed to be significant, a Repeated Measures ANOVA was performed for each of the seven clusters. The means and standard deviations for female and male subjects with relation to friend's gender are presented in Table 7-2. 
Table 7-2

Means and Standard Deviations for Number of Words Chosen from Each Cluster

\begin{tabular}{l|ll|ll}
\hline \hline & Male Subjects & $(\mathrm{n}=130)$ & $\begin{array}{l}\text { Female } \\
\text { Subjects }\end{array}$ & (n=151) \\
\hline & $\begin{array}{l}\text { same-sex } \\
\text { friends }\end{array}$ & $\begin{array}{l}\text { opposite-sex } \\
\text { friends }\end{array}$ & $\begin{array}{l}\text { same-sex } \\
\text { friends }\end{array}$ & $\begin{array}{l}\text { opposite-sex } \\
\text { friends }\end{array}$ \\
\hline $\begin{array}{l}\text { Cluster K } \\
\text { (Companionship) }\end{array}$ & $1.89(1.20)$ & $2.05(1.03)$ & $1.88(1.14)$ & $1.60(.98)$ \\
\hline $\begin{array}{l}\text { Cluster L } \\
\text { (Supportiveness) }\end{array}$ & $1.25(1.01)$ & $1.15(.90)$ & $1.46(1.01)$ & $1.52(1.07)$ \\
\hline $\begin{array}{l}\text { Cluster M } \\
\text { (Consideration) }\end{array}$ & $0.19(.43)$ & $0.47(.64)$ & $0.18(.43)$ & $0.26(.54)$ \\
\hline $\begin{array}{l}\text { Cluster N } \\
\text { (Integrity) }\end{array}$ & $0.60(.81)$ & $0.61(.73)$ & $0.48(.70)$ & $0.50(.69)$ \\
\hline $\begin{array}{l}\text { Cluster O } \\
\text { (independence) }\end{array}$ & $0.85(.93)$ & $0.54(.70)$ & $0.88(.91)$ & $0.93(.92)$ \\
\hline $\begin{array}{l}\text { Cluster P } \\
\text { (Open- } \\
\text { mindedness) }\end{array}$ & $0.09(.29)$ & $0.06(.24)$ & $0.05(.23)$ & $0.07(.25)$ \\
\hline $\begin{array}{l}\text { Cluster Q } \\
\text { (Respect) }\end{array}$ & $0.09(.29)$ & $0.05(.21)$ & $0.06(.24)$ & $0.09(.29)$ \\
\hline
\end{tabular}

Note. Standard deviations are in parentheses.

\section{Cluster K (Companionship)}

The Repeated Measures ANOVA revealed a significant main effect for subject's gender, $F(1)=4.00, p=.047$, with the mean score of males (1.95) being greater than that of females (1.76). No significant main effect for friend's gender was found.

A significant interaction effect for subject's gender by friend's gender was found, $F(1)=11.89, p=.001$. Paired $T$ Tests were performed and a significant effect was found for females, $\mathrm{p}=.002$, with the mean score for same-sex best friends (1.88) being greater than that of opposite-sex best friends (1.61). No significant effect was found for male subjects. 
Independent $\mathrm{T}$ Tests were performed and a significant effect was found for opposite-sex friends, $\mathrm{p}<.001$, with the mean score for male subjects $(2.05)$ being greater than that of female subjects (1.60). No significant effect was found for same-sex best friends.

\section{Cluster L (Supportiveness)}

The Repeated Measures ANOVA revealed a significant main effect for subject's gender, $F(1)=8.45, \mathrm{p}=.004$, with the mean score for males (1.21) being smaller than that of females (1.49). No significant main effect for friend's gender was found. No significant interaction effect for subject's gender by friend's gender was found.

\section{Cluster M (Consideration)}

The Repeated Measures ANOVA revealed a significant main effect for subject's gender, $\mathrm{F}(1)=4.71, \mathrm{p}=.031$, with the mean score for males (.33) being greater than that of females (.22). A significant main effect for best friend's gender, $F(1)=25.71, p<.001$, was found with the mean score for same-sex best friend (.18) being smaller than that of opposite-sex best friend (.36).

A significant interaction effect for subject's gender by friend's gender was found, $\mathrm{F}(1)=7.10, \mathrm{p}=.008$. Paired $\mathrm{T}$ Tests revealed a significant effect for males, $\mathrm{p}<.001$, with the mean score for same-sex best friend (.19) being smaller than that of opposite-sex best friend (.47). No significant effect was found for female subjects. Independent $\mathrm{T}$ Tests revealed a significant effect for opposite-sex best friends, $\mathrm{p}=.004$, with the mean score for male subjects 
(.47) being greater than that of female subjects (.26). No significant effect was found for same-sex best friends.

\section{Cluster N (Integrity)}

No significant main effect for subject's gender or friend's gender was found. No interaction effect for subject's gender by friend's gender was found.

\section{Cluster O (Independence)}

The Repeated Measures ANOVA revealed a significant main effect for subject's gender, $F(1)=5.80, p=.017$, with the mean score for males (.71) being smaller than that of females (.90). A significant main effect for best friend's gender, $F(1)=5.45, p=.020$, with the mean score for same-sex best friend (.87) being greater than that of opposite-sex best friend (.75) was found.

A significant interaction effect for subject's gender by friend's gender was found, $F(1)=10.00, p=.002$. Paired $T$ Tests revealed a significant effect for male, $\mathrm{p}<.001$, with the mean score for same-sex best friend (.85) being greater than that of opposite-sex best friend (.54). No significant effect was found for female subjects. Independent $T$ Tests revealed a significant effect for opposite-sex friends, $\mathrm{p}<.001$, with the mean score for male subjects $(.54)$ being smaller than that of female subjects (.93). No significant effect was found for same-sex best friends. 


\section{Cluster P (Open-Mindedness)}

No significant main effect for subject's gender or friend's gender was found. No interaction effect for subject's gender by friend's gender was found.

\section{Cluster Q (Respect)}

No significant main effect for subject's gender or friend's gender was found. A significant interaction effect was found, $F(1)=5.96, p=.015$. Paired T Tests were performed and a significant effect was found for males, $\mathrm{p}=.014$, with the mean score for same-sex best friend (.09) being greater than that of opposite-sex best friend (.04). No significant effect was found for female subjects. Independent-T Tests did not reveal significant effect for either same or opposite-sex best friends.

\section{Summary of the Results}

Overall significant main effects for subject's gender and for friend's gender and an overall significant interaction effect on subject's gender by friend's gender were reported. Specifically, in Cluster K (Companionship), the Repeated Measures ANOVA revealed a significant interaction effect for subject's gender by friend's gender. Paired T Tests found a significant effect for females, with the mean score for same-sex best friends (1.88) being greater than that of opposite-sex best friends (1.61). Independent $T$ Tests found a significant effect for opposite-sex friends, with the mean score for male subjects (2.05) being greater than that of female subjects (1.60). 
In Cluster L (Supportiveness), the Repeated Measures ANOVA revealed a significant main effect for subject's gender, with the mean score for males (1.21) being smaller than that of females (1.49).

In Cluster M (Consideration), the Repeated Measures ANOVA revealed a significant interaction effect for subject's gender by friend's gender. Paired T Tests revealed a significant effect for males, with the mean score for same-sex best friend (.19) being smaller than that of opposite-sex best friend (.47). Independent $\mathrm{T}$ Tests revealed a significant effect for opposite-sex best friends, with the mean score for male subjects (.47) being greater than that of female subjects (.26).

In Cluster N (Integrity), the Repeated Measures ANOVA revealed no significant main effect for subject's gender or friend's gender. No interaction effect for subject's gender by friend's gender was found.

In Cluster O (Independence), the Repeated Measures ANOVA revealed a significant interaction effect for subject's gender by friend's gender. Paired T Tests revealed a significant effect for males, with the mean score for same-sex best friend (.85) being greater than that of opposite-sex best friend (.54). Independent $\mathrm{T}$ Tests revealed a significant effect for opposite-sex friends, with the mean score for male subjects (.54) being smaller than that of female subjects (.93).

In Cluster P (Open-Mindedness), the Repeated Measures ANOVA revealed no significant main effect for subject's gender or friend's gender. No interaction effect for subject's gender by friend's gender was found.

In Cluster Q (Respect), the Repeated Measures ANOVA revealed a significant interaction effect for subject's gender by friend's gender. Paired 
$\mathrm{T}$ Tests were performed and a significant effect was found for males, with the mean score for same-sex best friend (.09) being greater than that of opposite-sex best friend (.04).

Discussion

\section{Overall Differences}

Two research questions were proposed in this stage:

RQ5: How does people's own gender influence their beliefs about satisfaction with best friends in Japan?

RQ6: How does people's best friend's gender influence their beliefs about satisfaction with these friends in Japan?

Based on the results of the Doubly Repeated Measures MANOVAs, overall differences can be summarized in the following three statements: (1) Japanese male and female subjects place different emphasis on the beliefs about relational satisfaction with best friends, (2) Japanese subjects place different emphasis on the beliefs about relational satisfaction depending on whether friends are same or opposite-sex, and (3) Japanese male and female subjects place different emphasis on the beliefs about relational satisfaction between male and female subjects with regard to the sex of the best friends.

In short, the results showed that both subject's and friend's gender have impact on their beliefs about relational satisfaction with best friends. More specific differences were investigated and discussed according to each of seven clusters. 


\section{Cluster K (Companionship)}

This cluster includes beliefs such as a best friend who "has similar values," who are "joyful to be with," and whom "I get along well with." The female subjects emphasized the quality of companionship more with female best friends than with male best friends. These findings are consistent with Sapadin's (1988) finding that women rated their same-sex friendships higher in intimacy and enjoyment than their opposite-sex friendships.

However, it is interesting to note that the findings also showed that the female subjects valued the quality of companionship with male best friends less than the male subjects did with female best friends. One implication of these results may be that the concept of companionship in best friendships is more universal to males regardless of best friend's gender than it is to female.

\section{Cluster L (Supportiveness)}

This cluster includes beliefs such as a person who is "caring," who "advises me," and whom "I can consult about anything." The findings suggest that Japanese female subjects want their best friends to be supportive more than male subjects do regardless of their best friends' gender. The findings are partially supported by previous research. Weiss and Lowenthal(1975) found that female friendships emphasize the importance of support. Caldwell and Peplau (1982) and Jourard (1971) reported that female friendships involve self-disclosure more than male friendships do. However, the present findings do not specify this quality as 
important only in female-female friendships but also in female-male friendships. These findings may be explained by the Japanese history of gender role orientation.

According to Sugisaki (1986), the position of Japanese women has been very low since the late sixth century from the influence of Buddhism and Confucianism until recent history. There has been a distinct shift upward in this position due to societal changes such as women's opportunities to become teachers since 1875 and the influence of the women's movement since 1879. However, the Confucian philosophy of women's "virtues of obedience and docility" (Sugisaki, 1986, p. 114) remains important in Japanese society even today. Raicho Hiratsuka, who published the first Japanese magazine run only by women wrote in 1911:

In the beginning a woman was the sun. She was a true human being. But now she is only the moon. Her life depends on others. She can shine only when reflecting light from others. Hers is the face of the moon, pale and sick. (cited in Sugisaki, 1986, p. 114)

Although the legal equality of women and men was assured by the new Japanese constitution in 1947, traditional gender roles created over a thousand years are still embedded deeply in Japanese culture and its people (Sugisaki, 1986; Sukemune, Shiraishi, Shirakawa, \& Matsumi, 1993). IshiiKuntz (1993) studied the role of Japanese fathers and reported that Japanese families still preserve and reinforce the traditional gender roles of "man as breadwinner and a woman as homemaker" (p. 61). Growing up in such an environment, the Japanese female subjects might acquire dependency 
more than male subjects and therefore, may value quality of support in friendships more.

\section{Cluster M (Consideration)}

This cluster includes beliefs such as consideration, calmness, and not being hypocritical. The male subjects emphasized this cluster more with female best friends than male best friends. The characteristic of this cluster is similar to the expectations of traditional Japanese women. The Confucian philosophy which was brought to Japan taught women to be obedient and docile (Sugisaki, 1986). This traditional role of Japanese women seems to persist in Japan. In her study on the cognitive structure of Japanese gender roles, Kashiwagi (cited in Shirakawa, Shiraishi, \& Sukemune, 1992) found "intelligence" and "activeness" as male gender role factors and "submissiveness with elegance" as a female gender role factor. She also found that the male subjects stressed "submissiveness with elegance" as an important factor for the female role. Therefore, it is not surprising that the male subjects emphasized the quality of consideration more from their female best friends than from their male. It is consistent with the fact that Confucian philosophy continues in contemporary Japanese society.

Interestingly, among the female subjects, the best friend's gender did not influence the emphasis of consideration to a significant degree. Two explanations are possible. One, the female subjects did not inherit as rigid a set of gender roles as male subjects because of the recent changes of women's status and opportunities. As a result, they expected as much 
consideration from men as from women, especially in best friendships. Or two, Japanese women may assume that the quality of consideration is inherent in Japanese females. Therefore, with no sense of a lack of this quality in other women, female subjects would not need to wish that their female friends possess it.

\section{Cluster $\mathrm{N}$ (Integrity)}

This cluster includes beliefs such as a best friend who "has common sense," who "has a sense of responsibility," and who is "not selfish." No gender differences in this cluster were found. This suggests that neither people's gender or their friends' gender do not affect the importance of integrity in Japanese friendships.

\section{Cluster O (Independence)}

This cluster includes beliefs such as a best friend who "has a sense of self," who is "a rival in a good sense," and who "has something I can learn." The male subjects emphasized this quality more from their male best friends than female best friends. This finding is consistent with Tannen's (1990) argument that women tend to focus more on intimacy while men tend to focus more on independence. According to Tannen's (1990), women live in a world of connection and men live in a world of status. The male subjects, who live in a world of status and value being independent, looked for this quality from their male best friends who are in the same world. 
Another explanation is the influence of Japanese traditional gender roles discussed earlier in this section. Having a long history of male supremacy, women are not expected to be independent but, rather, dependent or submissive (Sugisaki, 1986; Kashiwagi cited in Shirakawa, et al. 1992). The finding that the male subjects looked for this quality less in their female best friends than male best friends seems to reflect this gender role orientation.

However, the female subjects did not differentiate this quality depending on their friend's gender. This may be explained by recent changes of women's status in Japan. For instance, the Equal Employment Opportunity Act was passed in 1985 which called for equal pay and other opportunities in hiring and working conditions for women (Beasley, 1990; The Ministry of Education cited in Hirota, n.d.). In 1987, 37\% of Japanese women entered junior colleges or universities (The Ministry of Education cited in Hirota, n.d.). These societal changes may have influenced Japanese women about how they view themselves as well as others. As a result, the female subjects may no longer view the quality of independence as a unique male gender role, especially in a relationships with their best friends.

The male subjects emphasized this quality less in their female best friends than female subjects emphasized it in their male best friends. As noted earlier, men seem to value the quality of independence for men, while women seem to value this quality regardless to their friend's gender. Then, it is understandable that the male subjects did not look for this 
quality from their female friends as much as the female subjects did from their male friends

\section{Cluster P (Open-Mindedness)}

This cluster consists of one belief, "listens to a partner's opinion." No gender effect on emphasizing this cluster was found. This suggest that neither people's gender or their friends' gender do not affect the importance of open-mindedness in Japanese friendships.

\section{Cluster Q (Respect)}

This cluster consists of one belief, "does not look down on me." The male subjects emphasized this cluster more with their male best friends than female best friends. This male subjects' differentiation depending on their friend's gender may also be explained by Japanese traditional values as well.

Sugisaki (1986) argued that the Japanese social system has been "rigidly based on male supremacy" (p. 122), and "Japanese men have been traditionally so accustomed to treating women as their inferiors" (p. 122). Considering the claims above, the male subjects' choice of "not look down on me" as relevant to only male best friends might be explained by the male subjects subconsciously being aware that respect is something they have to earn from male friends but something which is inherent with female friends.

Another explanation can be made from the way traditional gender roles continue in Japanese families. According to Ishii-Kuntz (1993), many 
Japanese mothers described fathers as the "power" and "authority" to their children, especially sons, in order to give them a role model (p. 59). There may be some pressure for men to be respected like their father, especially by other men.

\section{Conclusion}

This stage explored gender influence on beliefs about relational satisfaction with best friends in Japan. The results showed that both subject's and friend's gender have some impact on beliefs concerning best friendships. Specifically, the following findings were revealed:

1) Female subjects emphasized the quality of Supportiveness more than male subjects did regardless of their friend's gender.

2) Female subjects emphasized the quality of companionship more with female best friends than with male best friends.

3) Male subjects emphasized the quality of consideration more with female best friends than with male best friends.

4) Male subjects emphasized the qualities of independence and respect more with male best friends than with female best friends.

5) There were no different emphasis on qualities of integrity or open-mindedness depending on subject's gender or their friend's gender. 
Some of these findings are consistent with the previous research in the U.S. The finding of the male subjects looking for the quality of independence with their male friends was explained by Tannen's (1990) insight about men focusing on independence. The finding that female subjects value companionship more with their female friends than with their male friends was consistent with Sapadin's (1988) findings. These cross-cultural consistencies suggest some similarities concerning gender influence on friendship between Japan and U.S.

Other findings were explained by Japanese cultural perspectives. The existence of Japanese traditional gender roles of women being submissive and men being authoritative was evident in the male subjects. However, the findings of female subjects were not always consistent with these gender roles. The reason why female subjects did not differentiate their concepts depending on their friends' gender as much as male subjects did was explained by recent changes of women's status and their influence on women's perception.

Two qualities, integrity and open-mindedness, were not influenced by either subjects' gender or their friends' gender. This implies that there are qualities people value in their best friendships beyond the gender roles.

There are several limitations in this stage. First, while each subject chose important words in relation to same-sex and opposite-sex best friends, these data may not be exactly comparable. According to the personal information subjects reported, many of the subjects did not have opposite-sex best friends in real life. In fact, 16 students did not choose the words for opposite-sex best friends because they did not have opposite-sex 
best friends or did not believe that opposite-sex best friends exist. These facts may affect the subjects' choices of important beliefs. For instance, their choices concerning same-sex best friends may be more realistic while the choice concerning opposite-sex best friends may be more ideal.

Second, when the subjects were asked to choose the important words for the opposite sex best friends, I did not direct them whether or not to think of non-romantic friendships. This decision was made based on the assumption that human relations are not mutually exclusive; some friendships may overlap with other relationships such as love or kin relationships. Therefore, it is not realistic to direct them to exclude friendships that include sexual attraction. However, Rands and Levinger (1979) claimed that subjects perceive cross-sex close friendships as love relationships. Therefore, differences presented here may not be based only on gender influence on friendship but also on different types of relationships. For instance, the subject's selection of words concerning the opposite sex best friends might be based on the beliefs about friendship as well as a possible love relationship.

Third, the differences related to a best friend's gender may be exaggerated. When people are asked the same question in different contexts, the question may highlight the difference of each context. In this study, subjects might emphasize gender differences more than they usually do because of the explicit focus on gender difference of friends.

Despite these limitations, the present stage raised several important aspects of the questions it posed in the field of friendship research as well as cultural research. First, there is a lack of research on gender influence in 
friendship in the cultures other than the U.S. This stage contributed to the gender research on friendships providing more information about Japanese friendship. Second, gender roles in Japan have mainly been studied historically, using anecdotes. Although this stage did not address gender roles directly, the implication of gender influence on Japanese friendship, using empirical methods, revealed some aspect of gender roles in modern Japanese culture.

Findings and limitations in this stage lead to two possible future directions. First, the subjects in this stage reported their ideas about friendships in general. As it was reported in the personal information, not many subjects had opposite-sex best friends in their real life. A study of several pairs of same-sex and opposite-sex best friends, using a qualitative method such as interviewing, will provide an opportunity to investigate whether the findings in this stage are born out in actual relationships.

Second, since this study may have magnified the differences between the two contexts, same-sex and opposite-sex best friendships, it will be useful to examine best friendships without specifying the sex of friends. A comparison of the concept of friendship between female and male subjects may not only resolve the limitation of this study but also reveal how a bias of the posed questions influences subject results. 
Chapter VIII

\section{Conclusion}

The present study consisted of five stages. Stage one through three explored Japanese people's beliefs about relational satisfaction with best friends by replicating Cole and Bradac's (1994) study. Stage four examined similarities and differences of organization of beliefs about relational satisfaction with best friends between Japan and the U.S. Stage five investigated gender influence on Japanese people's beliefs about satisfaction with best friends. In this chapter, summary of the findings, limitations and strengths of the study and future directions are presented.

\section{Summary of the findings}

In stage one, 39 Japanese people's perceived beliefs about relational satisfaction with best friends were identified. They include a best friend's characteristics (e.g., calm, big-hearted), attitudes (e.g., listens to a partner's opinion, advises me), and characteristics of the relationship (e.g., relationship that feels at ease, a give-and-take relationship). These 39 Japanese beliefs were compared with Cole and Bradac's (1994) 43 U.S. beliefs. Comparison of the beliefs revealed both similarities and differences between the two cultures. Many similarities are supported by previous researchers (e.g., Davis and Todd, 1985; La Gaipa, 1977). Two main differences were presented: 
1. Japanese subjects look for comfortable, predictable relationships rather than stimulating relationships, while U.S. subjects look for stimulating friendships rather than stable relationships.

2. Japanese tend to value the interdependent characteristic, specifically in-group responsibility, over the independent characteristic, while U.S. subjects value the independent characteristic over the interdependent characteristic.

These differences were explained by cultural tendencies of uncertaintyavoidance and individualism-collectivism.

Stage two introduced the idea that Japanese people's beliefs about relational satisfaction with best friends were organized in three underlying dimensions. These dimensions were interpreted as follows:

Dimension 1 as Light Hearted-Solidarity vs Serious-Rational (Kigaru, Rentaiteki Keikou vs Majime, Riseiteki Keikou), Dimension 2 as Acceptant-Reliable vs Independent-Positive (Juyou, Kakujitsuteki Keikou vs Jiritsu, Sekkyokuteki Keikou),

Dimension 3 as Frank-Supportive vs Modest-Self Controlled (Soccyoku, Shienteki Keikou vs Kenkyo, Jiseiteki Keikou). The findings suggest that Japanese people's beliefs are related to a best friend's (1) interpersonal-social desirable characteristics, (2) interdependent-independent dependability, and (3) interpersonal-social demeanor or active-passive support.

In stage three, the validity of interpretation of underlying dimensions of beliefs about satisfaction with best friends proposed in stage two was assessed. Dimensional interpretations for Dimension 1, Light 
Hearted-Solidarity vs Serious-Rational, and Dimension 2, AcceptantReliable vs Independent-Positive, were strongly supported as valid interpretations of proposed dimensions. The interpretation for Dimension 3, Frank-Supportive vs Modest-Self Controlled, was moderately supported.

In stage four, Japanese underlying dimensions of beliefs about relational satisfaction with best friends were compared to U.S. dimensions reported by Cole and Bradac (1994). The results suggest that both Japanese and U.S. people possess underlying structures concerning a best friend's interpersonal-social desirable characteristics and interdependentindependent dependability. The results also suggested that only U.S. people have an underlying dimension related to "a best friend's perceived level of activity" and only Japanese have an underlying dimension of a best friend's interpersonal-social demeanor or active-passive support.

In stage five, gender influence on Japanese people's beliefs about relational satisfaction with best friends was explored. The following findings were presented:

1. Female subjects emphasized the quality of supportiveness more than male subjects did regardless of their friend's gender.

2. Female subjects emphasized the quality of companionship more with female best friends than with male best friends.

3. Male subjects emphasized the quality of consideration more with female best friends than with male best friends. 
4. Male subjects emphasized the qualities of independence and respect more with male best friends than with female best friends.

5. There were no different emphases on qualities of integrity or open-mindedness depending on subject's gender or their friend's gender.

These findings imply some shared gender influences across cultures as well as culturally specific gender influences concerning friendships. The culturally specific gender influences were explained by traditional gender roles and recent changes of women's status and their influence on women's perception in the Japanese culture.

\section{Limitations and Strengths of the Study}

There are several limitations in this study. First, while using Japanese college students as a sample is appropriate for this study, Rands \& Levinger (1979) reported a generational influence on friendship. Therefore, although the findings of this study reflect Japanese people's beliefs about satisfaction with best friends, they specifically represent Japanese college students' beliefs.

Second, while some studies attempted to differentiate ideal friendships from actual friendships (e.g., Wish, Deutsch, \& Kaplan, 1976), this study did not address this issue. As it is assumed that people's beliefs about satisfaction with best friends reflect both their own and ideal friendships, this study took the approach of not specifying whether to 
think of one's own or ideal friendships. Because of this approach, it is predicted that some subjects thought more of their own friendships while others imagined more of their ideal friendships when participating in this study. Hence, it is unknown how subjects' responses based on their individual ways of thinking influenced the results of this study.

Third, comparison of my study to Cole and Bradac's (1994) study revealed cross-cultural differences between Japan and the United States. Based on these differences, I have argued that possible obstacles exist in developing intercultural friendships. However, Adler and Graham (1989) found that people use some different behaviors as a negotiation approach in an inter-cultural setting versus in an intra-cultural setting. They argued that cross-cultural differences observed from a comparative study might not be a problem in an inter-cultural setting, and warned against the use of cross-cultural differences as implications of intercultural problems.

Therefore, even though there are cross-cultural differences between Japanese and U.S people's beliefs, these differences may or may not be as crucial in an intercultural friendships as I have suggested.

There are several strengths in this study. First, in the field of friendship research, most studies have explored U.S. friendships while very few studies have examined Japanese friendships. Exploring Japanese people's beliefs about relational satisfaction with best friends, this study contributed to the friendship research providing more information about Japanese friendships.

Second, this study contained adequate size of samples in each stage. In addition, all samples were recruited in Japan and excluded Japanese 
students who seemed to have a certain level of intercultural experiences. Therefore, this study provided empirical data which have a minimum amount of contamination.

Third, this study did not use a pre-existing questionnaire constructed from a single cultural perspective. Instead, this study applied open-ended questions in stage one, asking subjects about their beliefs regarding satisfaction with best friends. The next questionnaire was then based on the results of stage one. This approach allowed for the emergence of beliefs which may be unique to Japanese people concerning friendships.

Fourth, many previous studies which used a multidimensional scaling technique reported the dimensional interpretation subjectively. This study examined the validity of proposed dimensional interpretations by using the same sample subjects. This process made the interpretation of Japanese underlying beliefs about relational satisfaction with best friends more credible.

\section{Future Directions}

There are several obvious directions that follow from the present study. First, there are few cross-cultural studies in friendship research. By replicating Cole and Bradac's (1994) study, this study revealed cross-cultural similarities and differences of beliefs about relational satisfaction with best friends between Japan and the U.S. Therefore, it would be useful to replicate Cole and Bradac's (1994) study with different cultural groups in 
order to explore similarities and differences of people's beliefs about relational satisfaction with best friends across cultures.

Second, this study specifically represents Japanese college students' beliefs about relational satisfaction with best friends. Replication of this study with people in different age groups as well as different status groups in Japan will help in understanding more about Japanese people's beliefs concerning friendships in general. It may also reveal age specific or status specific beliefs about satisfaction with best friends in Japan.

Third, even though Japanese and U.S. people share similar beliefs and similar organization of beliefs, it is unknown whether those beliefs mean the same between Japanese and U.S. people. Hence, it will be useful to explore what each belief means to Japanese and U.S. people. By doing so, results will reveal more descriptive behaviors and communication patterns based on people's beliefs about satisfaction with best friends. In turn, they will help to understand more subtle but salient cultural differences in beliefs related to relational satisfaction with best friends.

Fourth, this study is based on qualities that people think as important for friendships. It is unknown whether these qualities actually matter in their real friendships. A study of several pairs of best friends using a qualitative method such as interviewing will give an opportunity to investigate whether the beliefs emerged in this study really matter to Japanese people in their real relationships.

Fifth, cross-cultural differences between Japanese and U.S people's beliefs presented in this study may or may not be crucial in an intercultural friendship. Therefor, it is interesting to examine whether people's 
behavior as well as their interpretation of the friend's behavior changes depending on the cultural background of the best friend. One possible study for this examination would be a comparative study of inter-cultural best friends and intra-cultural best friends, using a qualitative method. This study may give some insight into how people's perceived similarity concerning cultural background influences their relationships with best friends. 
References

Adams, R. G., \& Bliezner, R. (1994). An integrative conceptual framework for friendship research. Iournal of Social and Personal Relationships, 11, 163-184.

Adelman, M. B., Parks, M. R., \& Albrecht, T. L. (1990). The nature of friendship and its development. In J. Stewart (Ed.), Bridges not walls. New York: McGraw-Hill Publishing.

Adler, N., \& Graham, J. (1989). Cross-cultural interaction: The international comparison fallacy? Journal of International Business Studies, 20, 515-537.

Albert, S. M. \& Moss, M. (1990). Consensus and the domain of personal relationships among older adults. Journal of Social and Personal Relationships, Z 353-369.

Allan, G. (1989). Friendship: Developing a sociological perspective. New York: Harvester Wheatsheaf.

Andersen, P. (1991). Explaining intercultural differences in nonverbal communication. In L. A. Samovar, \& R. E. Porter (Eds.), Intercultural communication: A reader (6th ed.). (pp. 286-296). Belmont, CA: Wadsworth.

Araki, H. (1973). Nihonjin no koudouyoushiki [Japanese pattern of behavior]. Tokyo, Japan: Kodansya.

Argyle, M., \& Henderson, M. (1985). The rules of friendships. In S. Duck and D. Perlman (Eds.), Understanding personal relationships: An interdisciplinary approach (pp. 63-84). Beverly Hills, CA: Sage. 
Aries, E. J., \& Johnson, F. L. (1983). Close friendships in adulthood: Conversational content between same-sex friends. Sex Roles, $9,1183-1196$.

Aukett, R., Ritchie, J., \& Mill, K. (1988). Gender differences in friendship patterns. Sex Roles, 19 , 57-66.

Babbie, E. (1992). The practice of Social research (6th ed.). Belmont, CA: Wadsworth.

Barnlund, D. C. (1989). Communication styles of Japanese and Americans: Images and realities. Belmont, CA: Wadsworth.

Barth, R. J., \& Kinder, B. N. (1988). A theoretical analysis of sex differences in same-sex friendships. Sex Roles, 19, 349-363.

Beasley, W. G. (1990). The rise of modern Japan. New York: St. Martin's Press.

Bell, R. R. (1981). Friendships of women and men. Psychology of Women Quarterly, $\underline{5}, 402-417$.

Black, H., \& Angelis, V. B. (1975). Sex role differences in the patterning of love among college students. Psychology, 12, 50-53.

Brein, M., \& David, K. H. (1971). Intercultural communication and the adjustment of the sojourner. Psychological Bulletin, 76(3), 215-230.

Burleson, B. R., \& Samter, W. (1990). Effects of cognitive complexity on the perceived importance of communication skills in friends. Communication Research, 17, 165-182

Caldwell, M. A., \& Peplau, L. A. (1982). Sex differences in same-sex friendship. Sex Roles, 8, 721-732.

Caroline, H. A. (1993). Explorations of close friendship: A concept analysis. Archives of Psychiatric Nursing, Z, 236-243. 
Cathcart, D., \& Cathcart, R. (1988). Japanese Social experience and concept of groups. In L. A. Samovar \& R. E. Porter (Eds.), Intercultural communication: A reader (5th ed.). (pp. 186-192). Belmont, CA: Wadsworth.

Cole, T., \& Bradac, J. (1994, July). A lay theory of relational satisfaction with best friends. Paper presented at the meeting of the International Communication Association, Sydney, Australia.

Davidson, L. R., \& Duberman, L. (1982). Friendship: Communication and interactional patterns in same-sex dyads. Sex Roles, 8(8), 809-822.

Davis, K. E., \& Todd, M. J. (1985). Assessing friendship: Prototypes, paradigm cases and relationship description. In S. Duck and D. Perlman (Eds.), Understanding personal relationships: An interdisciplinary approach (pp. 17-38). Beverly Hills, CA: Sage.

Davison, M. L. (1983). Multidimensional Scaling. New York: John Wiley \& Sons.

Devito, J. A. (1995). The interpersonal communication book (7th ed.). New York: Harper Collins College Publishers.

Dion, K. L., \& Dion, K. K. (1993). Gender and ethnocultural comparisons in styles of love. Psychology of Women Quarterly, 17, 463-473.

Driskill, G. W. (1995). Managing cultural differences: A rules analysis in a bicultural organization. The Howard Journal of Communications, 5, 353-379.

Duck, S. (1983). Friends for life. Sussex, England: Harvester Press. 
Duck, S., \& Wright, P. H. (1993). Reexamining gender differences in same-gender friendships: a close look at two kinds of data. Sex Roles, 28 , 709-727.

Elkins, L. E., \& Peterson, C. (1993). Gender differences in best friendships. Sex Roles, 29 497-508.

Endoh, K. (1995). The structure of hesitation in self-disclosure. Japanese. Journal of Counseling Science, 28 47-57.

Enomoto, H. (1987). Self-disclosure patterns of college students and their gender-differences in the patterns. Japanese Journal of Psychology 58(2), 91-97.

Eysenck, H. (1960). The place of theory in psychology. In H. Eysenck (Ed. ), Experiments in personality. London: Routledge \& Kegan Paul.

Fehr, B. (1996). Friendship processes. Thousand Oaks, CA: Sage.

Furnham, A. (1988). Lay theories: Everyday understanding of problems in the Social sciences. New York: Pergamon Press.

Furnham, A., \& Hesketh, B. (1987). Explanations for unemployment in Great Britain and New Zealand. The Journal of Social Psychology 129, 169-181.

Furnham, A., \& Trezise L. (1983). The mental health of foreign students. Social Science Med, 17, 365-370.

Gill, R., \& Keats, P. (1982). Elements of intellectual competence: Judgments by Australian and Malay university students. Lournal of CrossCultural Psychology, 11, 233-243. 
Goodwin, R., \& Lee, I. (1994). Taboo topics among Chinese and English friends: A cross-cultural comparison. Journal of Cross-Cultural Psychology, 25, 325-338.

Gudykunst, W. B., Yang, S, \& Nishida, T. (1985). A cross-cultural test of uncertainty reduction theory: Comparisons of acquaintances, friends, and dating relationships in Japan, Korea, and the United States. Human Communication Research, 11(3), 407-454.

Hacker, H. M. (1981). Blabbermouths and clams: Sex differences in self-disclosure in same-sex and cross-sex dyads. Psychology of women Quarterly, 5, 385-401.

Hair, J., Anderson, R. E., Tatham, R. L., \& Grablowsky, B. J. (1979). Multivariate data analysis. Tulsa, Oklahoma: Petroleum Publishing.

Hall, E. T. (1976). Beyond culture. Garden City, NY: Anchor Press.

Hall, E. T., \& Hall, M. R. (1990). Understanding cultural differences. Yarmouth, MN: Intercultural Press.

Hays, R. B. (1988). Friendship. In S. W. Duck (Ed.), Handbook of personal relationships (pp. 391-408). New York: John Wiley \& Sons.

Hecht, M. L., Andersen, P. A., \& Ribeau, S. A. (1989). The cultural dimensions of nonverbal communication. In M. K. Asante, \& W. B. Gudykunst (Eds.), Handbook of international and intercultural communication. (pp. 163-185). Newbury Park, CA: Sage.

Helman, C. (1984). Culture, health, and illness. Bristol: Wright.

Herzlich, C. (1973). Health and Illness. London: Academic Press.

Hirota, H. (n. d.). Facts about Japan: Japanese women today. Tokyo: The International Society for Educational Information. 
Homans, G. C. (1961) Social behavior: Its elementary forms. New York: Harcourt Brace \& World.

Hofstede, G. (1984). Culture's consequences, international differences in work-related values (Abridged Ed.). Beverly Hills, CA: Sage.

Hofstede, G. (1991). Cultures and organizations: Software of the mind. London: McGraw-Hill.

Infante, D. A., Rancer, A. S., \& Womack, D. F. (1993). Building communication theory (2nd ed.). Prospect Heights, IL: Waveland Press.

Ishii-Kuntz, M. (1993). Japanese fathers: work demands and family roles. In J. C. Hood (Ed.), Men, work, and family. (pp. 45-67) Newbury Park, CA: Sage.

Jaeger, R. (1990). Statistics: A spectator sport (2nd ed.). Newbury, CA: Sage.

Johnson, F. L., \& Aries, E. J. (1983). The talk of women friends. Women's Studies International Forum, 6 353-361.

Jourard, S. M. (1971). Self-disclosure: An Experimental analysis of the transparent self. New York: Wiley.

Keizai Koho Center. (1991). Japan 1992: An international comparison. Tokyo, Japan: Author.

Keller, H., Miranda, D., and Gauda, G. (1984). The naive theory of the infant and some maternal attitudes: A two-country study. Journal of CrossCultural Psychology, 15, 165-179.

Klein, R., Freeman, H., and Millett, R. (1973). Psychological test performance and indigenous conception of intelligence. Lournal of Social Psychology, 84, 219-222. 
Kluckhohn, F, Strodtbeck, F. (1961). Variations in value orientations. New York: Row, Peterson.

Kono, N., Maeda, E., Nishishiba, M., and Peterson, J. (1995, June). Lapanese and American concepts of friendship: A round-table report on an ongoing study. Paper presented at a regional conference of Asian Studies on the Pacific Coast, Forest Grove, OR.

Kruskal, J. \& Wish, M. (1978). Multidimensional Scaling. Sage University Paper series on Quantitative Applications in the Social Sciences, series no. 07-011. Beverly Hills, CA: Sage

La Gaipa, J. J. (1977). Testing a multidimensional approach to friendship. In S. Duck (Ed.), Theory and practice in interpersonal attraction. London: Academic Press.

Larwood, L., \& Wood, M. W. (1977). Women in management. Lexington: Lexington Books.

Lin, Y. W., \& Rusbult, C. E. (1995). Commitment to dating relationships and cross-sex friendships in America and China. Lournal of Social and Personal Relationships, 12, 7-26.

Lunt, P. K. (1991). The perceived causal structure of loneliness. Lournal of Personality and Social Psychology 61(1), 26-34.

Lunt, P. K., \& Livingstone. (1991). Everyday explanations for personal debt: A network approach. British Journal of Social Psychology, 30, 309-323.

Matsuura, H. (1991). An examination in mutual comparison of perception of equity in intimate pairs. The Japanese Journal of Experimental Social Psychology, 31(2), 155-166. 
Moghaddam, F. M., Taylor, D. M., \& Wright, S. C. (1993). Social psychology in cross-cultural perspective. New York: W. H. Freeman.

Moroi, K. (1989). An application of equity theory to interpersonal relations (2): Equity and emotional states in close relationships with samesex friends. The Japanese Journal of Experimental Social Psychology, 28(2), $131-141$.

Nakane, C. (1974). The Social system reflected in interpersonal communication. In J. C. Condon and M. Saito (Eds.), Intercultural encounters with Japan. Tokyo, Japan: The Simul Press.

Nakanishi, M. (1986). Perception of self-disclosure in initial interaction: A Japanese sample. Human Communication Research, 13, 167190.

Nakayama, K. (1992). Children's motivational orientations and their interactions with peers. Japanese Journal of Educational Psychology 40(4) 369-376.

Norusis, M. J. (1994) SPSS: SPSS advanced statistics 6.1. Chicago, SPSS Inc.

Oberg, K. Culture shock: Adjustment to new cultural environments. (1960). Practical Anthropology, $\underline{7}, 177-182$.

Okabe, R. (1983). Cultural assumptions of East and West. In W. B. Gudykunst (Ed.), Intercultural communication theory. (pp. 21-44). Beverly Hills, CA: Sage.

Okabe, R. (1987). Indirect speech acts of Japanese. In D. L. Kincaid (Ed.), Communication Theory: Eastern and Western perspectives. (pp. 127136) New York: Academic Press. 
Palisi, B. J., \& Ransford, H. E. (1987). Friendship as a voluntary relationship: Evidence from national surveys. Journal of Social and Personal Relationships, 4 , 243-259.

Pleck, J. H. (1975). Man to man: Is brotherhood possible? In N. Glazer-Malbin (Ed.), Old family/new family: Interpersonal relationships. New York: Van Nostrand.

Pogrebin, L. C. (1987). Among friends: Who we like, why we like them, and what we do with them. New York: McGraw-Hill.

Rands, M., \& Levinger, G. (1979). Implicit theories of relationship: An intergenerational study. Lournal of Personality and Social Psychology. $\underline{37}(5), 645-661$.

Rawlins, W. K., Leibowitz, K. \& Bochner, A. P. (1986). Affective and instrumental dimensions of best, equal, and unequal friendships. Central States Speech Journal 37 (2), 90-101.

Reisman, J. M. (1981). Adult friendships. In S. W. Duck \& R. Gilmour (Eds.), Personal relationships 2: Developing personal relationships (pp. 205-230). London: Academic Press.

Reohr, J. R. (1991). Friendship: An exploration of structure and process. New York: Garland Publishing, Inc.

Rose, S. M. (1985). Same- and cross-sex friendships and the psychology of homosociality. Sex Roles, 12, 63-74.

Ross, L. (1977) The intuitive psychologist and his shortcomings: Distortions in the attribution process. In L. Berkowitz (Ed.), Advances in Experimental Social psychology Vol. 10. New York: Academic Press. 
Rubin, L. B. (1986). Just friends: The role of friendship in our lives. New York: Harper \& Row.

Rubin, Z. (1973). Liking and loving: An invitation to Social psychology. New York: Holt, Rinehart \& Winston.

Ryoji Ijubu (1994, December). Ryoji iju kankei kiso deta [Basic data related to consulate immigration]. (Available from Consulate-General of Japan, Portland, 1300 SW 5th Ave. \#2400, Portland OR 97201).

Sapadin, L. A. (1988). Friendship and gender: Perspectives of professional men and women. Lournal of Personality and Social Relationships, $\underline{5}$, 387-403.

Sewell, W. H. \& Davidsen, O. M. (1960). Scandinavian students on an American campus. Minneapolis: University of Minnesota Press.

Sherrod, D. (1989). The influence of gender on same-sex friendships. In C. Hendrick (Ed.), Close relationships: Review of personality and Social psychology, Vol. 10 (pp. 164-186). Newbury Park, CA: Sage.

Shima, N. (1994). A study of Social support network measurement in high school students. Japanese Journal of Health Psychology 7(1), 14-25.

Shinmura, S. (1995). Pasokon ni yoru deta kaiseki [Data analyses using a personal computer]. Tokyo, Japan: Kodansya.

Shirakawa, Y, Shiraishi, T, \& Sukemune, S. (1992) Current research on gender roles in Japan. Psychologia, 35, 193-200.

Sternberg, S. J., Conway, B. E., Ketron, J. L. \& Bernstein, M. (1981). People's conceptions of intelligence. Lournal of Personality and Social Psychology $\underline{41}(1), 37-55$. 
Stewart, E. C. (1972). American cultural patterns: A cross-cultural perspective. Pittsburgh: Regional Council for International Education.

Storti, C. (1990). The art of crossing cultures. Yarmouth, ME: Intercultural Press.

Sugisaki, K. (1986). From the Moon to the Sun: Women's liberation in Japan. In L. Iglitzin \& R. Ross (Eds.), Women in the world, 1975-1985 (2nd rev. ed.). Santa Barbara, CA: ABC-Clio, Inc.

Sukemune, S., Shiraishi, T., Shirakawa, Y., and Matsumi, J. T. (1993). Japan. In L. L. Adler (Ed.), International handbook on gender roles. (pp. 174-186) London: Greenwood Press

Synder, M. \& Smith, D. (1986). Personality and friendship: The friendship worlds of self-monitoring. In V. J. Derlega \& B. A. Winstead (Eds.), Friendship and Social interaction. New York: Springer-Verlag.

Tannen, D. (1990). You just don't understand: Women and men in conversation. New York: Ballantine Books.

Taylor, E. W. (1994). Intercultural competency: A transformative learning process. Adult Education Quarterly, 44(3), 154-174.

Timmerman, G. M. (1991). A concept analysis of intimacy. Issues in Mental Health Nursing, 12, 19-30.

Ting-Toomey, S. (1989). Identity and interpersonal bonding. In M. K. Asante, \& W. B. Gudykunst (Eds.), Handbook of international and intercultural communication. (pp. 351-373). Newbury Park, CA: Sage.

Trenholm, S., \& Jensen, A. (1992). Interpersonal communication (2nd ed.). Belmont, CA: Wadsworth. 
Triandis, H. C. (1986). Collectivism vs individualism: A reconceptualization of a basic concept in cross-cultural psychology. In C. Bagley \& G. Verma (Eds.), Personality, cognition, and values: Cross-cultural perspectives of childhood and adolescence. London: Macmillan.

Triandis, H. C., Brislin, R., \& Hui, C. H. (1991). Cross-cultural training across the individualism collectivism divide. In L. A. Samovar \& R. E. Porter (Eds.), Intercultural communication: A reader (6th ed.). (pp. 370-382). Belmont, CA: Wadsworth.

Trice, A. D., \& Elliott, J. (1993). Japanese students in America: II. college friendship patterns. Iournal of Instructional Psychology, 20, 262-264.

U.S. Immigration and Naturalization Service (1985). Statistical Yearbook of the Immigration and Naturalization Service, 1984, Washington, D.C.: U.S. Government Printing Office.

U.S. Immigration and Naturalization Service (1994). Statistical Yearbook of the Immigration and Naturalization Service, 1993 , Washington, D.C.: U.S. Government Printing Office.

Verbrugge, L. M. (1977). The structure of adult friendship choices. Social Forces, $\underline{56}, 576-597$.

Walker, L. S., \& Wright, P. H. (1976). Self-disclosure in friendship. Perceptual and Motor Skills, 42, 735-742.

Weiss, L., \& Lowenthal, M. F. (1975). Life-course perspectives on friendship. In M. F. Lowenthal \& D. Chiriboga (Eds.), Four stages of life. San Francisco: Jossey-Bass.

Williams, D. G. (1985). Gender masculinity-femininity, and emotional intimacy in same-sex friendship. Sex Roles, 12, 587-600. 
Wish, M., Deutsch, M., \& Kaplan, S. J. (1976). Perceived dimensions of interpersonal relations. Journal of Personality and Social Psychology, $\underline{33}(4), 409-420$.

Won-Doornink, M. J. (1985). Self-disclosure and reciprocity in conversation: A cross-national study. Social Psychology Ouarterly, 48, 97107.

Wright, P. H. (1985). the acquaintance description form. In S. Duck and D. Perlman (Eds.), Understanding personal relationships: An interdisciplinary approach (pp. 39-62). Beverly Hills, CA: Sage.

Young, J. E. (1986). A cognitive-behavioral approach to friendship disorders. In V. J. Derlega \& B. A. Winstead (Eds.), Friendship and Social interaction. New York: Springer-Verlag.

Yum, J. O. (1991). The impact of Confucianism on interpersonal relationships and communication patterns in East Asia. In L. A. Samovar \& R. E. Porter (Eds.), Intercultural communication: A reader (6th ed.). (pp. 66-78). Belmont, CA: Wadsworth. 
Appendix A

Informed Consent Form 


\section{Informed Consent}

I, (please print your name), agree to take part in this research project on Japanese people's beliefs about satisfaction with best friends, conducted by Eriko Maeda, a graduate student of Portland State University.

In this research, the following conditions apply: I will answer questions related to friendships, especially your idea of friendship, my name will be kept confidential, all information I give will be kept confidential to the extent required by law, I may not receive any direct benefit from taking part in this study but the study may help to increase knowledge that may help others in the future, my participation is voluntarily and I can refuse to answer any questions in the questionnaire or I can stop my involvement at any time. I have read and understand the above information and agree to take part in this study.

Date: Signature:

If you have concerns or questions about this study, please contact Eriko Maeda at 0792-35-4312 (Japan), Dr. David Ritchie in the Dept. of Speech Communication at 503-725-3550 (U.S.A.), or the Office of Research and Sponsored Projects at Portland State University at 503-725-3417 (U.S.A.). 
Appendix B

English Translation of the Questionnaires 


\section{Questionnaire One}

This is a part of a research project on concepts of best friendship for the Japanese. In this questionnaire, I ask what are important things for you in order to have a satisfying relationship with a best friend. Therefore, there are no right or wrong answers for all the following questions. Please write down your ideas in the way you like. This questionnaire consists of four pages. Please answer pages in order starting from the first page.

1. What is a best friend for you? Please think how you would like your best friend to act towards you and the types of qualities you would like a best friend to have. After giving this some thought, please write down all of the characteristics and qualities you would like your best friend to have in order to have satisfying relationship with him/her. In other words, I would like you to describe 1) what you want your best friend to be like, 2) what you want a best friend to do. You may write in a word or a sentence. You do not need to fill in all the blanks below. If you have more than 15 responses, please feel free to use the space of this page or back of this page.

1.

2.

3.

4.

5.

6.

7.

8.

9.

10.

11.

12.

13.

14.

15. 
2. What makes a relationship with a best friend dissatisfying? Please think about what a dissatisfying friendship would be like. Please think how the other person would act towards you and the types of qualities they would have in this type of relationship. After giving this some thought, please write down all of the characteristics and qualities that would make a relationship with a best friend dissatisfying. In other words, I would like you to describe 1) what the other person would be like, and 2) what they would do to make your relationship with them dissatisfying and unenjoyable. You may write in a word or a sentence. You do not need to fill in all the blanks below. If you have more than 15 responses, please feel free to use the space of this page or back of this page.

\section{1.}

$\underline{2 .}$

3.

4.

5.

6.

7.

8.

2.

10.

11.

12.

13.

14.

15. 
3. Please give some thought again to what is important for you in order to make a satisfying relationship with a best friend. Then, please complete the sentences below by filling in blanks. It is okay to create a sentence that does not sound fluent or to change the sentence itself.

(Example: "A best friend should do okay.) ." --> "A best friend should do write." is

A best friend should be

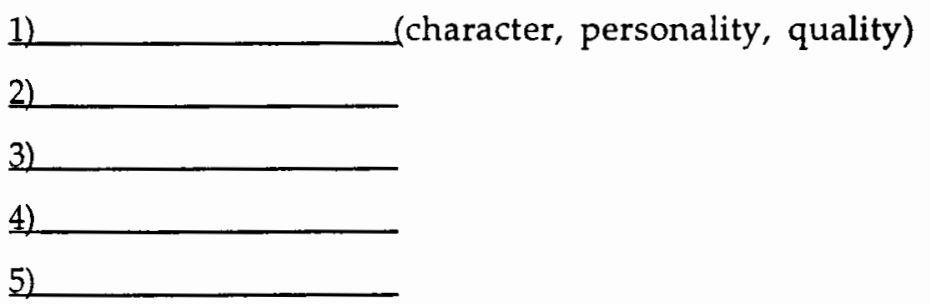

A best friend should not be

1) (character, personality, quality).

2)

3)

4)

5)

A best friend should do

$$
\text { 1) }
$$
(behavior, verb, action).

2)

3)

4)

5)

A best friend should not do

1) (behavior, verb, action).

2)

3)

4)

5) 


\section{Questionnaire Two}

This questionnaire is for a research project on Japanese people's beliefs about satisfaction with best friends, conducted by Eriko Maeda, a graduate student of Portland State University.

In this research, the following conditions apply:

1. You will answer questions related to friendships, especially your idea of friendship.

2. Your name will be kept confidential.

3. all information you give will be kept confidential to the extent required by law.

4. You may not receive any direct benefit from taking part in this study. But the study may help to increase knowledge that may help others in the future.

5. Your participation is voluntarily and you can refuse to answer any questions in the questionnaire or you can stop your involvement at any time.

After you have read and understand the above information and agree to take part in this study, please move on to the next page and begin with question \#1.

If you have concerns or questions about this study, please contact Eriko Maeda at 0792-35-4312 (Japan), Dr. David Ritchie in the Dept. of Speech Communication at 503-725-3550 (U.S.A.), or the Office of Research and Sponsored Projects at Portland State University at 503-725-3417 (U.S.A.). 
1. This research explores how people conceptualize characteristics and qualities that people want their best friends to have. These characteristics and qualities are printed on a set of 39 cards.

Directions:

1. Please spread all the cards on a table.

2. Pleas read the characteristics on each cards.

3. Please group the cards into piles based on how similar the characteristics are to each other.

Cautions: 1 . There is no limitation about numbers of groupings.

2. You can make a grouping from one card.

3. You can regroup the cards.

4. Please use all the cards for grouping.

5. You can use each card only once.

4. After you have grouped all of the cards, please look over your piles one more time and make sure that the characteristics in each pile are similar for you.

5. For each group, please transcribe the number on the upper right side of each card on to the lines below.

Remember to change lines every time you move to a different group.

For this task, I am interested in which characteristics you think are similar. There are no right or wrong grouping. The only rule I want you to follow is that the characteristics which belong to the same group are similar for you.

A:

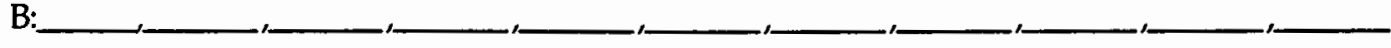

C:

D.

E:

F:

G:

$\mathrm{H}$ :

I:

J:

K 
The characteristics and qualities people want their best friends to have

\begin{tabular}{|c|c|c|}
\hline $\begin{array}{l}\text { 1. Does not depend on a friend } \\
\text { too much }\end{array}$ & 14. Not selfish & $\begin{array}{l}27 . \text { A person I can have a long- } \\
\text { term relationship with }\end{array}$ \\
\hline 2. Listens to a partner's opinion & 15. Has a sense of self & 28. A person I can consult about \\
\hline 3. A rival in a good sense & $\begin{array}{l}\text { ot brag about } \\
\text { aff }\end{array}$ & 29. Encourages me \\
\hline 4. Joyful to be with & 17. Has common sense & $\begin{array}{l}\text { 30. Deals with } \\
\text { seriously whe }\end{array}$ \\
\hline 5. Does $\mathrm{n}$ & 18. & 31. Does not speak ill of others \\
\hline 6. Co & & 32. Po \\
\hline $\begin{array}{l}\text { 7. Says what he/she thinks } \\
\text { clearly }\end{array}$ & worthy & 33. $\mathrm{Ag}$ \\
\hline 8. Calm & $\begin{array}{l}\text { 21. Straightforward/Not } \\
\text { hypocritical }\end{array}$ & 34. Does not pry to \\
\hline 9. Has similar values & 22. Has a sense of respons & $\begin{array}{l}\text { 35. A relationship in which we } \\
\text { keep in contact with each other }\end{array}$ \\
\hline $\begin{array}{l}\text { 10. A person I get along well } \\
\text { with }\end{array}$ & 23. Treats everyone equal & 36. Has something I can learn \\
\hline $\begin{array}{l}\text { 11. Relationship that feels at } \\
\text { ease }\end{array}$ & 24. Advises me & 37. Has similarities with me \\
\hline $\begin{array}{l}\text { 12. Can confide in } \\
\text { 13. Big-hearted }\end{array}$ & $\begin{array}{l}\text { ociable } \\
\text { person I spend a long time }\end{array}$ & $\begin{array}{l}\text { 38. Understands me } \\
\text { 39. Does not look down }\end{array}$ \\
\hline
\end{tabular}

2. Please take a look at the list above which are the characteristics and qualities people want their best friends to have. Please choose five of them in the order of importance that you want the other person to have in order to have a satisfying relationship with the same sex best friend. Then, please write down the number of these characteristics. If you cannot choose characteristics from the list, please circle [0] and write down the reason if possible.

$1.2 \underline{2 .} \underline{3 .} \underline{5 .}$

0 . When I think of a relationship with the same sex best friend, I cannot choose characteristics from the list.

Reason

3. From the list which are the characteristics and qualities people want their best friends to have, please choose five of them in the order of importance that you want the other person to have in order to have a satisfying relationship with a opposite sex best friend. Then, please write down the number of these characteristics. If you cannot choose characteristics from the list, please circle [0] and write down the reason if possible.

$\underline{1 .} \underline{2 .} \underline{3 .} \underline{4 .}$

0 . When I think of a relationship with a opposite sex best friend, I cannot choose characteristics from the list.

Reason 
Questionnaire 3-A

This questionnaire is for a research project on Japanese people's beliefs about satisfaction with best friends, conducted by Eriko Maeda, a graduate student of Portland State University.

In this research, the following conditions apply:

1. You will answer questions related to friendships, especially your idea of friendship.

2. Your name will be kept confidential.

3. all information you give will be kept confidential to the extent required by law.

4. You may not receive any direct benefit from taking part in this study. But the study may help to increase knowledge that may help others in the future.

5. Your participation is voluntarily and you can refuse to answer any questions in the questionnaire or you can stop your involvement at any time.

After you have read and understand the above information and agree to take part in this study, please move on to the following instructions.

If you have concerns or questions about this study, please contact Eriko Maeda at 0792-35-4312 (Japan), Dr. David Ritchie in the Dept. of Speech Communication at 503-725-3550 (U.S.A.), or the Office of Research and Sponsored Projects at Portland State University at 503-725-3417 (U.S.A.).

\section{Instructions}

This research explores the characteristics and qualities people want their best friends to have. This particular questionnaire examines how people evaluate these characteristics and qualities in terms of three different dimensions. Specifically, I would like you to evaluate each of 20 characteristics and qualities in terms of the following criteria:

1) whether they are light-hearted-solidarity or serious-rational,

2) whether they are acceptant-reliable or independent-positive,

3) whether they are frank-supportive or modest-self-controlled.

As these evaluations are very unusual, I would like you to answer by referring to the detailed explanation of each dimension as well as examples of each characteristic (which are described in the parentheses of each characteristic).

There are no right or wrong answers. In this questionnaire, I am simply interested in which tendency you think each characteristic strongly possesses. Please make a decision based on what you feel intuitively. 
1. Please evaluate each of the following 20 characteristics according to whether it is light-hearted-solidarity or serious-rational. Light-heartedsolidarity refers to being light-hearted, comfortable, easy to get along, and solidarity. On the other hand, serious-rational refers to being serious, sincere, rational, and superior.

Light-hearted-solidarity refers to being... being...

$$
\begin{aligned}
& \text { light-hearted } \\
& \text { comfortable } \\
& \text { easy to get along with } \\
& \text { solidarity }
\end{aligned}
$$

\section{Serious-rational refers to}

$$
\begin{aligned}
& \text { serious } \\
& \text { sincere } \\
& \text { rational } \\
& \text { superb }
\end{aligned}
$$

Please evaluate whether each characteristic strongly possesses lighthearted-solidarity or serious-rational tendencies. In terms of evaluation, please circle one of the five if a characteristic:

clearly illustrates the idea of being light-hearted-solidarity -- 1

somewhat illustrates the idea of being light-hearted-solidarity -- 2

does not illustrates either tendency strongly -- 3

somewhat illustrates the idea of being serious-rational-- 4

clearly illustrates the idea of being serious-rational-- 5

There is no right or wrong answers. Please make a decision based on what you feel intuitively.

1. Does not depend on a friend too much

light-hearted-solidarity

$$
1
$$

2

3

serious-rational

(Aite ni izon shisuginai)

(can take care of him/herself, does not depend on others too much)

2. Listens to a partner's opinion

1

(Aite no iken wo kiku)

(listens to my story, does not force his/her opinion)

3. A rival in a good sense

(Ii imi deno raibaru de aru)

(a good rival, we stimulate each other, stimulative)

4. Joyful to be with

(Issyoni ite tanoshii)

(we enjoy together, a happy person, a cheerful person, fun)

5. Does not lie

(Uso wo tsukanai)

(does not keep things back, honest, faithful) $\begin{array}{lllll}1 & 2 & 3 & 4 & 5\end{array}$

$\begin{array}{lllll}1 & 2 & 3 & 4 & 5\end{array}$

$\begin{array}{lllll}1 & 2 & 3 & 4 & 5\end{array}$ 


\section{Considerate}

light-hearted-solidarity

\section{(Omoiyari ga aru)}

12

serious-rational

(can understand how other people feel, kind,

has consideration, does not do spiteful things to others)

7. Says what he/she thinks clearly

1

(Omotteirukoto wo hakkiri iu)

(tells what he/she thinks, says things clearly)

8. Calm (Onkou de aru)

12

(not short-tempered, gentle)

9. Has similar values (Kachikan ga niteiru)

(has similar ideas, has the same value)

10. A person I get along well with (Ki ga au)

(we are on the same wave-length, we have similar sensitivity, we have the same sense of humor)

\section{Relationship that feels at ease}

(Ki wo tsukawanai kankei de aru)

(frank, I can relax when being with him/her,

we can spend time without conversation)

12. Can confide in (Kuchi ga katai)

12

(holds a secret, does not talk to other people what I consulted to him/her)

13. Big-hearted (Kokoro ga hiroi)

(tolerant, does not worry about details)

14. Not selfish (Jibun katte de nai)

(cooperative, not self-centered, not egoistic)

15. Has a sense of self

(Jibun to iumono wo motteiru)

(has his/her own idea, has his/her own belief,

not a follower, not indecisive)

16. Does not brag about him/herself

(Jiman wo shinai)

(does not always brag about him/herself,

does not behave self-important)

17. Has common sense (Joushiki ga aru)

(behaves according to circumstances,

has good Social manners, does not do things against morals)

18. Sensible (Shiryo bukai)

(cool, not too excitable, does not behave hastily)

19. Caring (Shinmi ni natte kureru)

(concerns about me, thinks about me seriously)

20. Trustworthy (Shinrai dekiru)

(reliable, a person who I can rely on, does not let me down) $\begin{array}{llll}1 & 2 & 3 & 4\end{array}$

5

$\begin{array}{lllll}1 & 2 & 3 & 4 & 5\end{array}$

$\begin{array}{lllll}1 & 2 & 3 & 4 & 5\end{array}$

$\begin{array}{llll}1 & 2 & 3 & 4\end{array}$

5

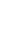


2. Please evaluate each of the following 20 characteristics according to whether it is acceptant-reliable or independent-positive. Acceptant-reliable refers to being dependable, and reliable. On the other hand, independentpositive refers to being independent mentally, firm, positive, and active.

Acceptant-reliable refers to being... being...

broad-minded
warm-hearted
dependable
reliable

\section{Independent-positive refers to}

independent mentally firm positive active

Please evaluate whether each characteristic strongly possesses acceptantreliable or independent-positive tendencies. In terms of evaluation, please circle one of the five if a characteristic

clearly illustrates the idea of being acceptant-reliable -- 1

somewhat illustrates the idea of being acceptant-reliable -- 2

does not illustrates either tendency strongly -- 3

somewhat illustrates the idea of being independent-positive -- 4

clearly illustrates the idea of being independent-positive -- 5

There is no right or wrong answers. Please make a decision based on what you feel intuitively.

1. Does not depend on a friend too much

$$
\text { acceptance-reliable }
$$

(Aite ni izon shisuginai)

(can take care of him/herself, does not depend on others too much)

2. Listens to a partner's opinion

(Aite no iken wo kiku)

(listens to my story, does not force his/her opinion)

3. A rival in a good sense

(Ii imi deno raibaru de aru)

(a good rival, we stimulate each other, stimulative)

4. Joyful to be with

(Issyoni ite tanoshii)

(we enjoy together, a happy person, a cheerful person, fun)

5. Does not lie

(Uso wo tsukanai)

(does not keep things back, honest, faithful)
12

independent-positive

345

$\begin{array}{lllll}1 & 2 & 3 & 4 & 5\end{array}$

$\begin{array}{lllll}1 & 2 & 3 & 4 & 5\end{array}$

$\begin{array}{lllll}1 & 2 & 3 & 4 & 5\end{array}$

$\begin{array}{lllll}1 & 2 & 3 & 4 & 5\end{array}$ 
3. Please evaluate each of the following 20 characteristics according to whether it is frank-supportive or modest-self-controlled. Light-heartedsolidarity refers to being light-hearted, easy to get along, and solidarity. On the other hand, serious-rational refers to being serious, sincere, rational, and superior.

Frank-supportive refers to being... being...

frank

definite

supportive

lead me

\section{Modest-self-controlled refers to}

modest

reserved

self-controlled

restrained

Please evaluate whether each characteristic strongly possesses franksupportive or modest-self-controlled. In terms of evaluation, please circle one of the five if a characteristic

clearly illustrates the idea of being frank-supportive -- 1

somewhat illustrates the idea of being frank-supportive -- 2

does not illustrates either tendency strongly -- 3

somewhat illustrates the idea of being modest-self-controlled -- 4

clearly illustrates the idea of being modest-self-controlled -- 5

There is no right or wrong answers. Please make a decision based on what you feel intuitively.

controlled

1. Does not depend on a friend too much (Aite ni izon shisuginai) (can take care of him/herself, does not depend on others too much)

2. Listens to a partner's opinion

(Aite no iken wo kiku)

(listens to my story, does not force his/her opinion)

3. A rival in a good sense

(Ii imi deno raibaru de aru)

(a good rival, we stimulate each other, stimulative)

4. Joyful to be with

(Issyoni ite tanoshii)

(we enjoy together, a happy person, a cheerful person, fun)

5. Does not lie

(Uso wo tsukanai)

(does not keep things back, honest, faithful)

1

1

frank-supportive

modest-self-

2

34

5

$4 \quad 5$ 
Questionnaire 3-B

This questionnaire is for a research project on Japanese people's beliefs about satisfaction with best friends, conducted by Eriko Maeda, a graduate student of Portland State University.

In this research, the following conditions apply:

1. You will answer questions related to friendships, especially your idea of friendship.

2. Your name will be kept confidential.

3. all information you give will be kept confidential to the extent required by law.

4. You may not receive any direct benefit from taking part in this study. But the study may help to increase knowledge that may help others in the future.

5. Your participation is voluntarily and you can refuse to answer any questions in the questionnaire or you can stop your involvement at any time.

After you have read and understand the above information and agree to take part in this study, please move on to the following instructions.

If you have concerns or questions about this study, please contact Eriko Maeda at 0792-35-4312 (Japan), Dr. David Ritchie in the Dept. of Speech Communication at 503-725-3550 (U.S.A.), or the Office of Research and Sponsored Projects at Portland State University at 503-725-3417 (U.S.A.).

\section{Instructions}

This research explores the characteristics and qualities people want their best friends to have. This particular questionnaire examines how people evaluate these characteristics and qualities in terms of three different dimensions. Specifically, I would like you to evaluate each of 19 characteristics and qualities in terms of the following criteria:

1) whether they are light-hearted-solidarity or serious-rational,

2) whether they are acceptant-reliable or independent-positive,

3) whether they are frank-supportive or modest-self-controlled.

As these evaluations are very unusual, I would like you to answer by referring to the detailed explanation of each dimension as well as examples of each characteristic (which are described in the parentheses of each characteristic).

There are no right or wrong answers. In this questionnaire, I am simply interested in which tendency you think each characteristic strongly possesses. Please make a decision based on what you feel intuitively. 
1. Please evaluate each of the following 19 characteristics according to whether it is light-hearted-solidarity or serious-rational. Light-heartedsolidarity refers to being light-hearted, comfortable, easy to get along, and solidarity. On the other hand, serious-rational refers to being serious, sincere, rational, and superior.

Light-hearted-solidarity refers to being... being...

Serious-rational refers to

light-hearted
comfortable
easy to get along with
solidarity
serious
sincere
rational
superb

Please evaluate whether each characteristic strongly possesses lighthearted-solidarity or serious-rational tendencies. In terms of evaluation, please circle one of the five if a characteristic:

clearly illustrates the idea of being light-hearted-solidarity -- 1 somewhat illustrates the idea of being light-hearted-solidarity -- 2 does not illustrates either tendency strongly -- 3 somewhat illustrates the idea of being serious-rational-- 4 clearly illustrates the idea of being serious-rational-- 5

There is no right or wrong answers. Please make a decision based on what you feel intuitively.

\section{Straightforward/Not hypocritical}

light-hearted-solidarity

(Sunao de aru):

(does not have a split/double face. apologizes when he/she is wrong)

2. Has a sense of responsibility

$$
12
$$

3

4

5

(Sekininkan ga aru):

(has a strong sense of responsibility, not irresponsible, keeps his/her promise)

3. Treats everyone equal

(Dare ni demo byoudou de aru):

(does not change his/her attitude depending on people, does not judge people according to their past)

$\begin{aligned} & \text { 4. Advises me (Cyuukoku shite kureru): } \\ & \text { (tells me when I am wrong, sometimes stern, points out my mistakes) }\end{aligned}$
$\begin{aligned} & \text { 5. Sociable (Tsukiai ga ii): } \\ & \text { (can play with me when I want) }\end{aligned}$


Appendix $\mathrm{C}$

Instruction for Stage One 


\section{Instruction}

1. Tell the students that this questionnaire is a part of the research on "Japanese concept of a best friendship" by Eriko Maeda, a graduate student at Portland State University and that you agreed to help Maeda's research.

2. Distribute the two copies of the informed consent form for each student. One is to submit me, and the other is for themselves in case they can reach me when they have any comments or questions. ( I think this is a standard procedure for the research like mine.)

3. Instruct the students to sign their names in two spaces and the date in one space.

4. Collect the informed consent forms. Then give the information below:

1) This is not a quiz.

2) There is no time limit to fill in the questionnaire.

3) Do not write the students' name.

4) Submit the questionnaire in the envelop or box on the front desk (if there is any front desk) when finish writing.

5) The result of this research will be presented next year. If the students would be interested in the result, they should sign their names, addresses, and phone numbers on the request form when they submit the questionnaire.

6) If the students are willing to be interviewed by Eriko Maeda around Oct. '95, they should also sign their names, addresses, and phone numbers on the same request form above.

5. Distribute the questionnaires to the students. Then, read the first paragraph of the questionnaire aloud if possible. (From "Koreha Nihonzin no ....." to "junnban ni shitsumon ni kotaeteitte kudasai.") After this, let them start writing.

6. After collecting all the questionnaires, please mail the questionnaires, consent forms, and request form together by either express mail or one of the courier services.

Note: It is important to collect informed consent forms and to keep the questionnaires confidential. although the time the students use to fill in the questionnaire varies, it will probably take 30 minutes. 


\section{Other Instructions}

1. As it is said in the informed consent form, the students' participation is voluntarily. If a student asks you whether it is okay not to participate in the study, you should say "yes," then ask the student to leave the classroom.

2. If there are students who cannot finish writing within the class time, it is okay to tell them to finish it up later and submit it to you as soon as possible like the next day.

3. although there is an instruction to fill in the questionnaire from the first page, it is okay if a student would go back to add something in the first page when he/she is working on the second page. (The instruction is given because of the concern about the influence of the questions in the last page to the other questions.)

4. If there is a question from the students whether it is okay to have the same answer in page three as page one or two, please answer that it is okay to have the same answers as well as the different answers.

5. If a student asks you whether it is okay to fill in more than five or less than five answers in the page three, please tell them that it is okay. if they have more than five, tell them to use any space on the page or on the back of the page. 
Appendix D

Personal Information 
I. Please answer the following questions about yourself.

1. Age:

2. Sex: 1 . male

2. female one)

3. Year in college:

4. Major:

5. Have you ever lived abroad?

1. yes

2. no (please circle one)

6. If you answer "yes" to question 5, please write down the places and the length of living overseas.

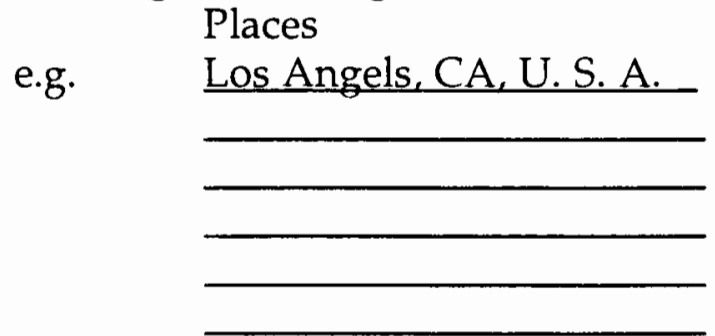

Lengths

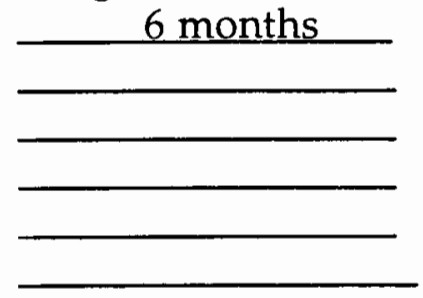

II. Please answer the following questions about your friends.

1. How many best friends do you have?

2. Please list ages, sex, and the way you and your friends become acquainted. acquainted

Age Sex The way you and your friends become

e.g. 21 male We were classmates in the high school.

3. Do you have best friends who are not Japanese residents?

1. Yes 2. No

(please circle one) 
Appendix E

Cole and Bradac's (1994) Perceived Beliefs about Relational Satisfaction with Best Friends 
1. Accepting/Supportive: Accepts me unconditionally. Completely supports the things I do and is not critical of my lifestyle, values, ideas, and feelings.

2. Active/Energetic: The active adventurous type. Is bold, daring, and risk taker.

3. Activities Director: Comes up with creative activities, introduces me to others, and always invites me to do things.

4. Admits Mistakes: Has no problems admitting when they are wrong or makes mistakes.

5. altruistic: Is always willing to help me when I need it. Makes sacrifices for me, tries to make me happy, and is willing to share their possessions with me.

6. Ambitious: Motivated and very goal oriented. An achiever.

7. Appreciates Me: Enjoys my company and is truly interested in me. Values our friendship and is always excited to see me.

8. Approachable: always willing and easy to talk to. Isn't aloof or distant.

9. Available: Has plenty of time to spend with me and is always willing to make time for me.

10. Caring, Kind, and Compassionate: Understanding, empathetic, warm, forgiving, sensitive, and thoughtful.

11. Committed: Acknowledges our friendship; is willing to make me a part of their life. Interested in a long term involvement with me.

12. Complementary : Possesses attributes that I lack. Their personality picks up where mine leaves off. They have qualities that I don't have.

13. Confident: Possesses a lot of self-respect. Very secure, assertive, and decisive.

14. Creative: Is a little off beat, unique, and holds interesting ideas.

15. Dependable: Keeps their word. Is trustworthy, punctual, and doesn't break promises.

16. Doesn't Use Me: Doesn't try to take advantage of me, manipulate me, or mooch off me.

17. Easy Going: Very free spirited, relaxed, and light hearted.

18. Emotionally Balanced: Is emotionally stable. Is not moody, too sentimental, or bad tempered. Doesn't overreact.

19. Equal: Is of equal status. Doesn't worship me or dominate me; rather they are on my same level. 
20. Family Oriented: Has traditional family values, a good family background, and is family oriented.

21. Flexible: Not set in their ways and are open to new ideas. They are adaptive and able to change and grow.

22. Fun to be Around: Has a great sense of humor and is always fun to be with. Very entertaining and likes to joke around.

23. Good Communication Skills: Knows how to handle conflicts, has good listening skills, and always knows how and when to say things the right way.

24. Happy: Very positive and optimistic.

25. Healthy: Is athletic. Takes care of their body and doesn't abuse alcohol and drugs.

26. Honest: always tells the truth. Very genuine with me.

27. Independent: Has a life of their own. Has their own ideas, friends, and goals. Isn't jealous, overly needy or dependent on me.

28. Inspiring: Is my role model. Inspires me to be a better person, gives me advice, and makes me think about my ideas.

29. Intelligent: Has an education and is smart.

30. Intimate: Shares their secrets with me. Knows me well and I also know their innermost ideas.

31. Loyal: Defends me in front of others. Does not try to steal my friends, talk behind my back, or betray me.

32. Not a Whiner: Doesn't dump their problems on me. Able to handle their own problems and stress and doesn't complain to me.

33. Not Greedy: Isn't excessively materialistic.

34. Not Physically Violent: Is not a violent person.

35. Not Self-Absorbed: Doesn't always talk about him/herself. Is not a bragger, conceited, or ego-centric.

36. Not Verbally Abusive: Is not mean, sarcastic, patronizing, or argumentative. Doesn't say things to anger me.

37. Open Minded: Is liberal. Isn't prejudiced or critical of others.

38. Outgoing/Friendly: Very charismatic, social, friendly, and liked by all.

39. Physically Attractive: Is good looking, clean, and well groomed. 
40. Practical: Has a lot of common sense. Is down to earth, realistic, and mature.

41. Respects Me: Respects my feelings, privacy, and property. Trusts and believes in me.

42. Serious: Is quiet, deep, and thinks a lot. Not shallow or superficial.

43. Share Similarities: Has many things in common with me. Has similar values, morals, interests, career goals, and political views. Shares my sense of humor and likes to do the same things that I do.

Note. From "A lay theory of relational satisfaction with best friends," by T. Cole and J.

Bradac, 1994, Paper presented at the meeting of the International Communication

Association, Sydney, Australia. 
Appendix F

Questionnaires and Personal Information Survey in Japanese 


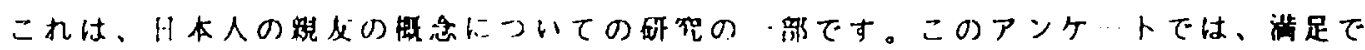

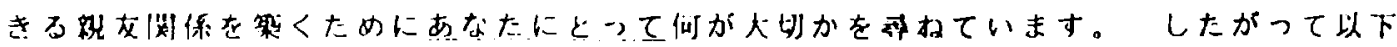

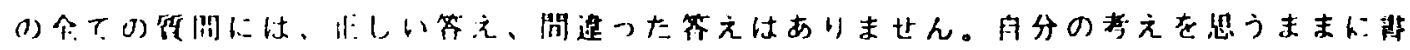

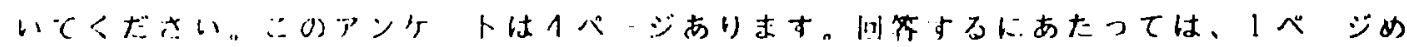

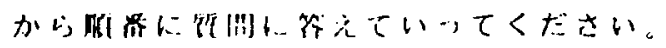

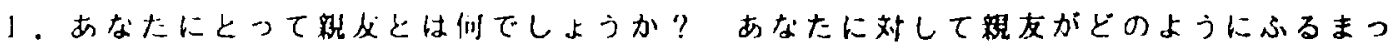
てほしいか、どのような特微があるかを考えてみてください。その上で、視友との関俰を 满足させるために相手がもつベき特微や性買を思いつく限り简条書きで以下に書いてくだ さい。つまり、親友との関保を染しく满足できるものにするためには、1）䚅友がどんな 人であってほしいか、2) 親友にどんなことをしてほしいかを春いてください。書き方は 単語でも、文でも、かまいません。全ての空梶をうめる必要もありません。もし15 項目 以上ある訝合は、このページの余白または奥面を使ってください。

\begin{tabular}{l}
1. \\
2. \\
3. \\
\hline 4. \\
\hline 5. \\
6. \\
7. \\
\hline 8. \\
\hline 0. \\
\hline 1. \\
\hline 2. \\
\hline 3. \\
\hline 5. \\
\hline
\end{tabular}




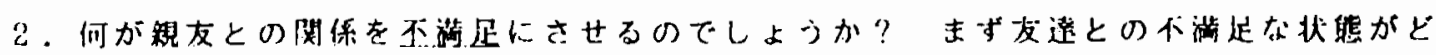

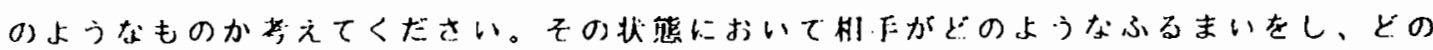

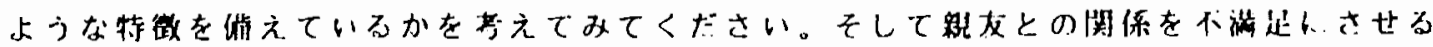

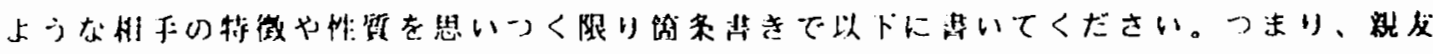

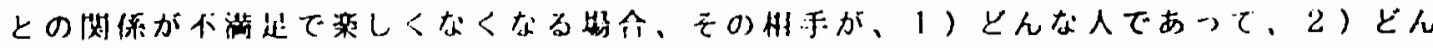

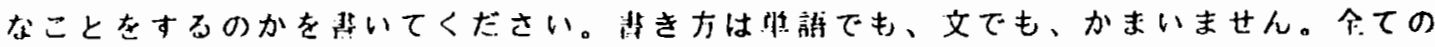

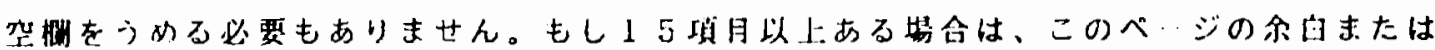
面を使つてください。

\begin{tabular}{l}
1. \\
2. \\
3. \\
4. \\
5. \\
6. \\
7. \\
8. \\
9. \\
10. \\
11. \\
12. \\
\hline 13. \\
14. \\
\hline 15.
\end{tabular}


3.もう一度、满足できる親友関係を筑くためにあなたにとって何が大切か考えてくださ い。そして以下の空概に自由にことはを入れて、文章を完成させてください。空构に轱く 文算少変わってもかまいません。

(例、 をするべきです - - -> 毛蚳を書く するべきです。としても可。）

耤友は、上) （性格、人柄、特性）であるべきです。

2.)

3)

4)

5)

親友は、1) (性格、人柄、特性)であるべきではありません。

2)

3)

4)

5)

親友は、1) （行糼、野菏、言動）をするべきです。

2)

3)

4)

52)

親友は、1)

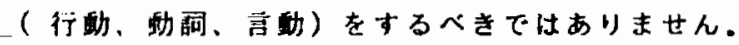

2)

32

4.)

5) 
彩友の樶念について 研究 2

この筫問用紙は、ボートランド州立大学、大学院生、前田江里子の行なっている

「日本人の親友の概念について」の研究のためのものです。

この研究において.

1、あなたは友情、特に親友についてあなたの考えに基づいて算問えます。

2、あなたの名前はどのような形であっても公表されることはありません。

3、全ての解答は匿名で扱われます。

4、この研究に参加することによって直接の利益を受けないか、この研究は将来 人々に役立つ知戴に賁献しています。

5、この研究の参加は強制ではなく、又参加をいつでもやめることができます。

以上の内容を玩み理解した上で、この研究に参加することを同意した場合、次のべージ に進み、䇤問】から順番に解答してください。

尚、この研究について意見、問のある場合は，前田江里子 (0792-35-4312)、Dr. David Ritchie (米国503-725-3550)、又はボートランド州立大学の Office of Research and Sponsored Projects (米国503-725-3417)に連絡してくたさい。 


\section{新友の概念ついて 研究 2}

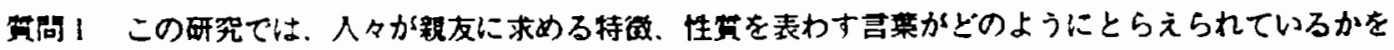

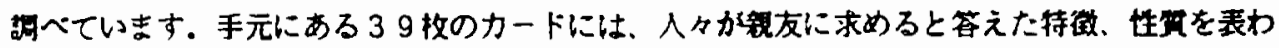
す言莱が書かれています。

手原1. 全てのカードを机に広姑てください.

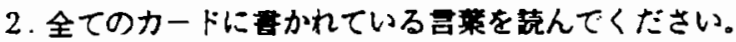

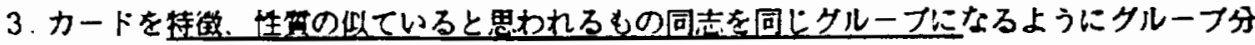
けしてくださ。

这 1：グルーフの数に制限はありません.

注 $2 ：$ 寻聚がつしかないタルーフがあってもかまいません。

注 3 : ダルーフ訜けしている内、カードを何度入九替えてもかまいません.

注 $4:$ 全ての乔栔をグルーブ分けしてくだい。

注 5：1つの㤟莱注1つのクループにしか使えません。

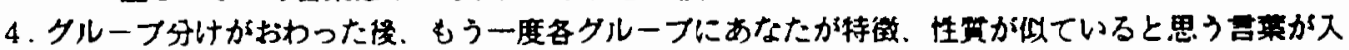
っているかを礁かめてください。

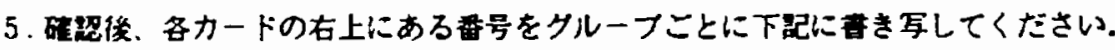

書き写寸際、クルーフことに行を变えることを忘机ないてください。

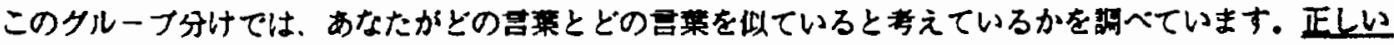

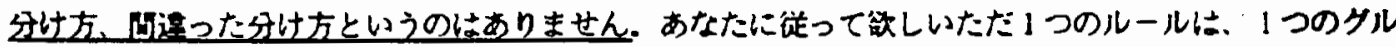
ーブにスる言莱はお互いに似ているということです。

A :

B :

$c:$

$\mathrm{D}:$

E :

F :

G :

H :

I :

J :

K : 
剆友の概念について 研究 2

人々が親友に求めている特徴、性算

\begin{tabular}{|c|c|c|}
\hline 1、相手に在存しすぎない & 14、自分暸手でない & 27、長いつきあいかできる \\
\hline 2、相手の竟見を間く & \multicolumn{2}{|c|}{ 15．自分というものをもっている 28 ，何でも相腅できる } \\
\hline 3、いい寻朱でのライハルである & 16、自傮をしない & 29．助ましてくれる \\
\hline 4、一腥にいて渠しい & 17. 常蓸がある & 30．必晨な時に直面目に对応してくれる \\
\hline 5、うそをつかない & 18，思漹深い & 3 1、人の慁口を豆わない \\
\hline 6、思いやクがある & 19、䚀身になってくれる & 32、前向きである \\
\hline \multicolumn{2}{|c|}{ 7、思っていることをはっきり寻つ 20 ．信頼できる } & 33、もちつもたれつの閣保である \\
\hline 8. 温厚である & 21. 声であろ & 34 、余計なことを詮索しない \\
\hline 9、洒值旣が似ている & 2 2、佘任䲷がある & 35，運䊀し合う \\
\hline 10．気が合う & 2 3、誰にでも平等である & 36、私が学べる何かをもっている \\
\hline 11、気を使わない成保である & 24、忠告してくれる & 37、私と共通した点がある \\
\hline 12、口か整い & 25.つきあいがいい & 38.私のことを理解している \\
\hline 13、心が広い & 26、其に違こしたた時閌長し & 39、私を見下さない \\
\hline
\end{tabular}

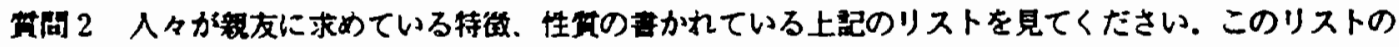

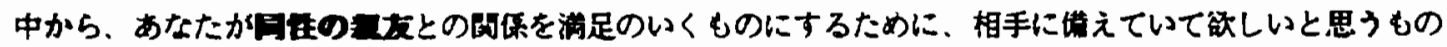

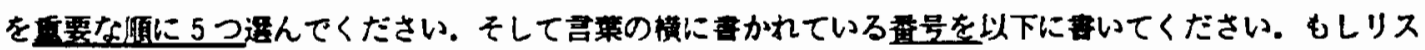

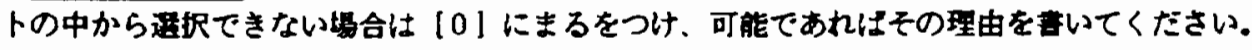

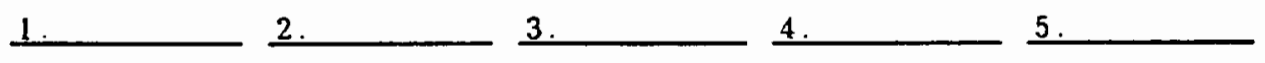

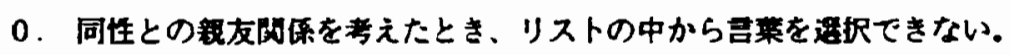

理由:

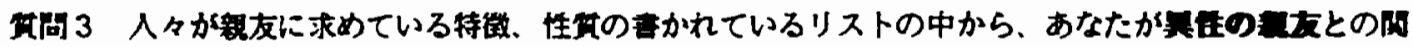

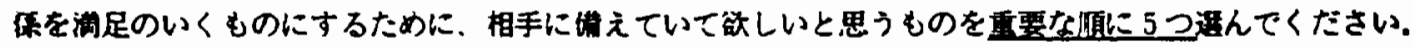

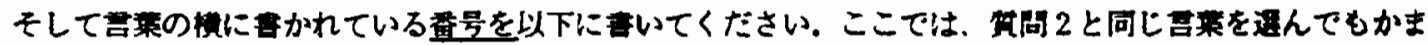

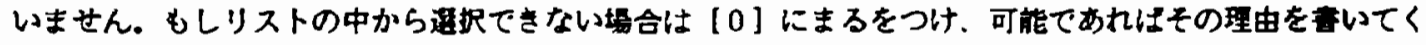
ださ.

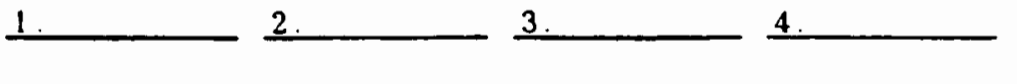

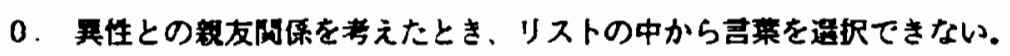

理由： 


\section{親友関係を淍足にきせる概念について}

この闆用紙は、ポートランド州立大学、大学院生、前田江里子の行なっている

「日本人の親友の概念について」の研究のためのものです。

この研究において.

1、あなた洁友情、特に親友についてあなたの考えに基づいて賏問に答えます。

2、あなたの名前はどのような形であっても公表されることはありません。

3、全ての解答は蒀名で扱わ机ます。

4、この研究に参加することによって直接の利益を受けないか、この研究は将来 人々に役立つ知識に貢献しています。

5、この研究の参加は強制ではなく、㟥加をいつでもやめることができます。 以上の内容を読み理解した上で、この研究に参加することを同意した場合、以下の説明 を繶けて芜み、指示にしたがってください。

尚、この研究について意見、筫問のある場合は、前田江里子 (0792-35-4312)、Dr. David Ritchie（米国503-725-3550). 又汴ボートランド州立大学の Office of Research and Sponsored Projects (米国503-725-3417)に連絡してください。

\section{部明}

この研究では、人々が親友に求めている特徽、性筫を考察しています。このアンケート

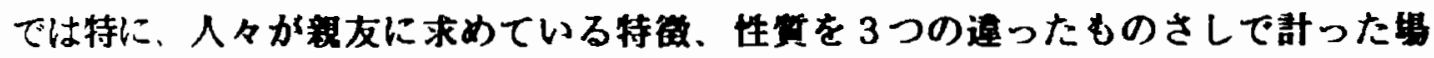
合どのように評佃されるかを考察しています。具体的に言えば、人々が親友に求めてい ると思われる 20 項目の特徴。性兴それぞれが

1）気轻、連帯的傾向であるか、まじめ、理性的傾向であるか、

2）受容、確実的傾向であるか、自立、䞍電的傾向であるか、

3）率直、支援的傾向であるか、謙虚、自制的傾向であるか、

$$
\text { という評価をしていただきます。 }
$$

こういった䓄問は大変特殊なものなので、各べージに書かれたそれそれの傾向の詳しい 説明および、人々が親友に求めている特徽、性の例文（各特徵、性の下のカッコ中に 記述）を十分参考にして回答してください。

この筫問には正しい答え、間遑った答えというのはありません。このアンケートは、 あなたがそれぞれの特徵、性にどちらの頱向が強いと考えるかを知るためのものです。 ですから回答するにあたっては、あなたの思うように直感で判断してください。それでは 始めてください。 
親友関保を渵足にささる概念について

1、以下にあげる久々が親友に求めている 20 項目の特徽、性筫を、気轻、連带的㥧向 であるか、まじ、理性的㥧向であるかというものさしに従って評価してください。 気軽、連帯的傾向と漳しく言えは、気軽な、気楽な、つき合いやすい，連帯感のあるに 関係しており、ましめ、理性的傾向とは詳しく言えは、ましめな、誠実な、理性的な、優 れたに関倸しています。

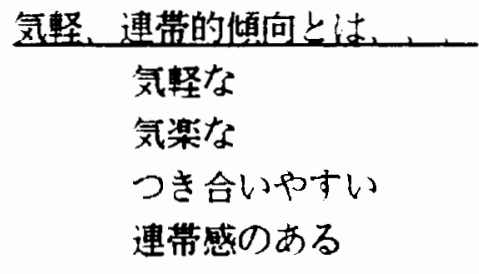

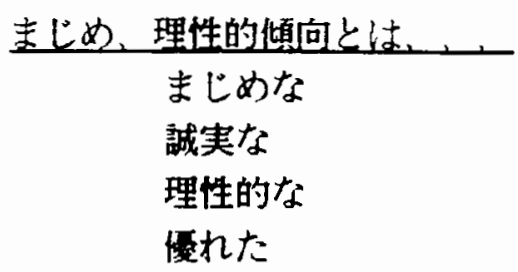

あなたにとって、それそれれの特徽が気堑、連帯的傾向、ましめ、理性的傾向どちらの傾 向をより強く備えているかを評価してください。評価にあたっては、以下の評価の仕方に 従って1から5のうちいずれか1つにマルをつけてください。

明らかに気軽、連带的傾向を示していると思うーー1

少し気軽、連带的傾向を示していると思うーー 2

どちらとも言えないと思うーー 3

少しましめ、理性的㑯向を示していると思うーー4

明らかにましめ。理珄的傾向を示していると思うーー5

この筫問には正しい答え、間量った答えというのはありません。回答するにあたって は、あなたの思うように直感で判断してください。

気柽、尰蒂的攧向

ましめ、理性的值向

1、相手に依存しすきない

(自分のことがきちんとできる、他人に頼りすぎい

\section{2、相手の意見を開く}

(話を䦳いてくれる、自分の意見を押しつ!なない

3、いい意味でのライハルである

(よきライバル，お互いを高め合う、刺激的)

4、一腥にいて㷊しい

(楽しい人、明るい人、おもしろい)

5、うそをつかない

(僖し事をしない，正直、誠実な人) 
親友関係を渵足にさせる概念について

2、以下にあげる人々が親友に求めている 20 項目の特徵、性を、受容、䒠的烦向 であるか、自立、精医的㑯向であるかというものさしに従って評価してください。受 容、確実的傾向とは詳しく言えは、包容力がある、人情のある、頼れる、あてになるに関 保しており、自立、皘極的傾向とは詳しく言えは、精神的に自立した、しっかりした、皘 極的な、活発なに関係しています。

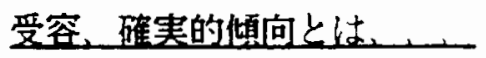 \\ 包容力がある \\ 人情のある \\ 頼れる \\ あてになる
}

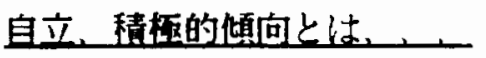 \\ 精神的に自立した \\ しっかりした \\ 皘極的な \\ 活発な
}

あなたにとって、それそれれの特徵が受容、確実的㥧向、自立、䞍䡮的傾向どちらの攧向 をより強く備えているかを評洒してください。評洒にあたっては、以下の評価の仕方に従 って1から5のうちいずれか1つにマルをつけてください。

明らかに受容、確実的傾向を示していると思うーー1

少し受容、確実的傾向を示していると思うー- 2

どちらとも言えないと思うーー 3

少し自立、皘極的愐向を示していると思うーー4

明らかに自立、皘極的傾向を示していると思うーー 5

この問には正しい答え、間遑った答えというのはありません。回答するにあたって は、あなたの思うように直感で判断してください。

\section{受容、確実的专问}

1、相手に依存しすきない

(w)

2、相手の意見を闑く

地人に顐りすきなない

(話を閍いてくれる、自分の意見を押しつけない)

\section{3、いい意味でのライバルである}

(よきライハル、お互いを高め合う、刺潄的)

\section{4、一粕にいて楽しい}

(楽しい人、明るい人、おもしろい)

5、うそをつかない

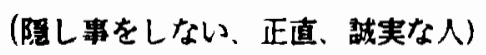

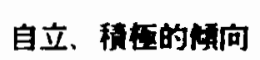

$\begin{array}{llll}2 & 3 & 4 & 5\end{array}$

$\begin{array}{llll}2 & 3 & 4 & 5\end{array}$

$\begin{array}{llll}2 & 3 & 4 & 5\end{array}$

$\begin{array}{llll}2 & 3 & 4 & 5\end{array}$

$\begin{array}{lllll}1 & 2 & 3 & 4 & 5\end{array}$ 
3、以下にあげる人々が親友に求めている 20 項目の特徵、性筫を、率直、支搌的煩向 であるか、望虐、自韧的煩向であるかというものさしに従って評価してください。率 直、支援的傾向とは詳しく言えば、率直な、はっきりと、支援的な、引っぱってくれるに 関係しており、謙虚、自制的傾向とは詳しく言えは、譜虚な、ひかえめな、自制心のある、 おさえたに関倸しています。

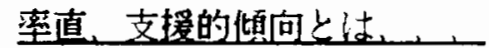

率直な

はっきりと

支援的な

引っばってくれる

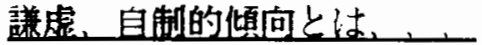

謙虚な

ひかえめな

自制心のある

おさえた

あなたにとって、それぞれの特徵が率直、支嗳的傾向、謙虚、自制的傾向どちらの傾向 をより強く備えているかを評洒してください。評洒にあたっては、以下の評価の仕方に従 っていから5のうちいずれか1つにマルをつけてください。

明らかに率直、支授的傾向を示していると思うーー1

少し率直、支授的傾向を示していると思うーー 2

どちらとも言えないと思うーー 3

少し謙虚、自制的傾向を示していると思うーー 4

明らかに謙虚、自制的傾向を示していると思うーー5

この問には正しい答え、間違った答えというのはありません。回答するにあたって は、あなたの思うように直感で判断してください。

平直、支授的攧向

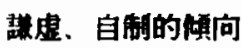

1、相手に依存しすきない

$\begin{array}{lllll}1 & 2 & 3 & 4 & 5\end{array}$

（自分のことがきちんとできる、他人に頼りすぎない)

2、相手の意見を閜く

(話を閶いてくれる、自分の意見を押しつけない)

3、いい意味でのライバルである

(よきライバル、お互いを高め合う、刺激的)

4.一楮にいて楽しい

(楽しい人，明るい人，おもしろい)

5、うそをつかない

(隐し事をしない，正直、諴実な人) 
親友関係を満足にさせる概念について

I. 以下の問に答えてください。

1. 年令

3. 学部 / 学科

5 ，海外に滞在したことがありますか。

6.5で「はい」と答えた人は、海外での漟在地域と期間を書いてください。 （3 筒所以上ある場合は、このページの余白を使ってください。） 滞在地

附

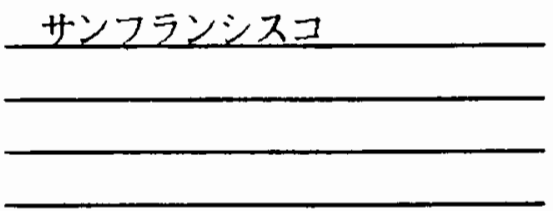

2. 性別

4. 学年

はい
6 力月

男性

$$
\text { いい⿱亠乂 }
$$

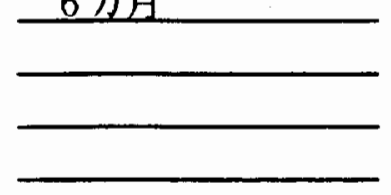

女性

II. あなたとあなたの親友についての、以下の筫問えてください。

1. あなたが親友と思える人は何人いますか。

2. あなたが親友と思える人の現在の年令、性別、どのく゚らいの期間その人を知ってい

るか、そしてどのようにしてあなたがその人と知り合いになったかを書いてくださ W.

(6 人以上いる場合は、このぺージの余白を使ってください。)

年令 性別 知っている期間 知り合いになった方法

例 21 男 4 年 耷校で同じうラスだった

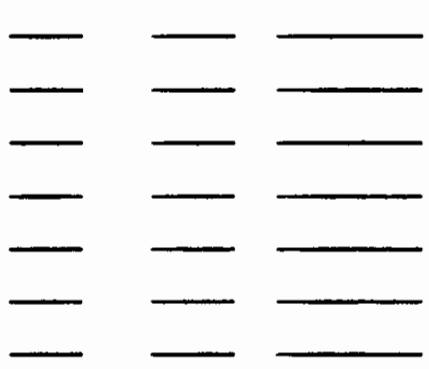

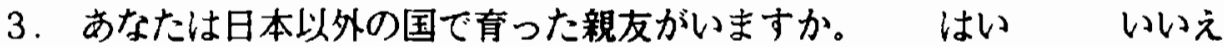

4.3で「はいと答えた人は、その親友の育った国名を、2の表の横に書きたしてく ださい。 